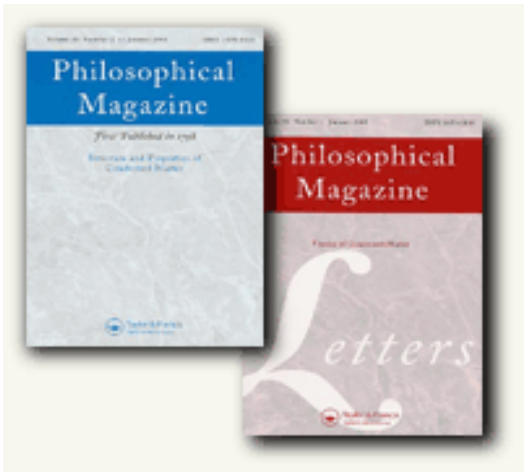

\title{
Exchange interactions, spin waves and transition temperatures in itinerant magnets
}

\begin{tabular}{|c|c|}
\hline Journal: & Philosophical Magazine \& Philosophical Magazine Letters \\
\hline Manuscript ID: & TPHM-05-Jun-0279.R1 \\
\hline Journal Selection: & Philosophical Magazine \\
\hline $\begin{array}{r}\text { Date Submitted by the } \\
\text { Author: }\end{array}$ & 20-Oct-2005 \\
\hline Complete List of Authors: & $\begin{array}{l}\text { Turek, Ilja; Institue of Physics of Materials, Academy of Sciences } \\
\text { Kudrnovsky, Josef; Institute of Physics, Acadeny of Sciences } \\
\text { Drchal, Vaclav; Institute of Physics, Academy of Sciences } \\
\text { Bruno, Patrick; MPI for Microstructure Physics }\end{array}$ \\
\hline Keywords: & $\begin{array}{l}\text { magnetization dynamics, magnetic phase transition, magnetic } \\
\text { properties, magnetic structure, magnetic transitions, magnetism, } \\
\text { first-principles calculations }\end{array}$ \\
\hline \multicolumn{2}{|l|}{ Keywords (user supplied): } \\
\hline \multicolumn{2}{|c|}{$\begin{array}{l}\text { Note: The following files were submitted by the author for peer review, but cannot be converted } \\
\text { to PDF. You must view these files (e.g. movies) online. }\end{array}$} \\
\hline nvr2.tex & \\
\hline
\end{tabular}

\section{(5)holarONE \\ Manuscript Central}



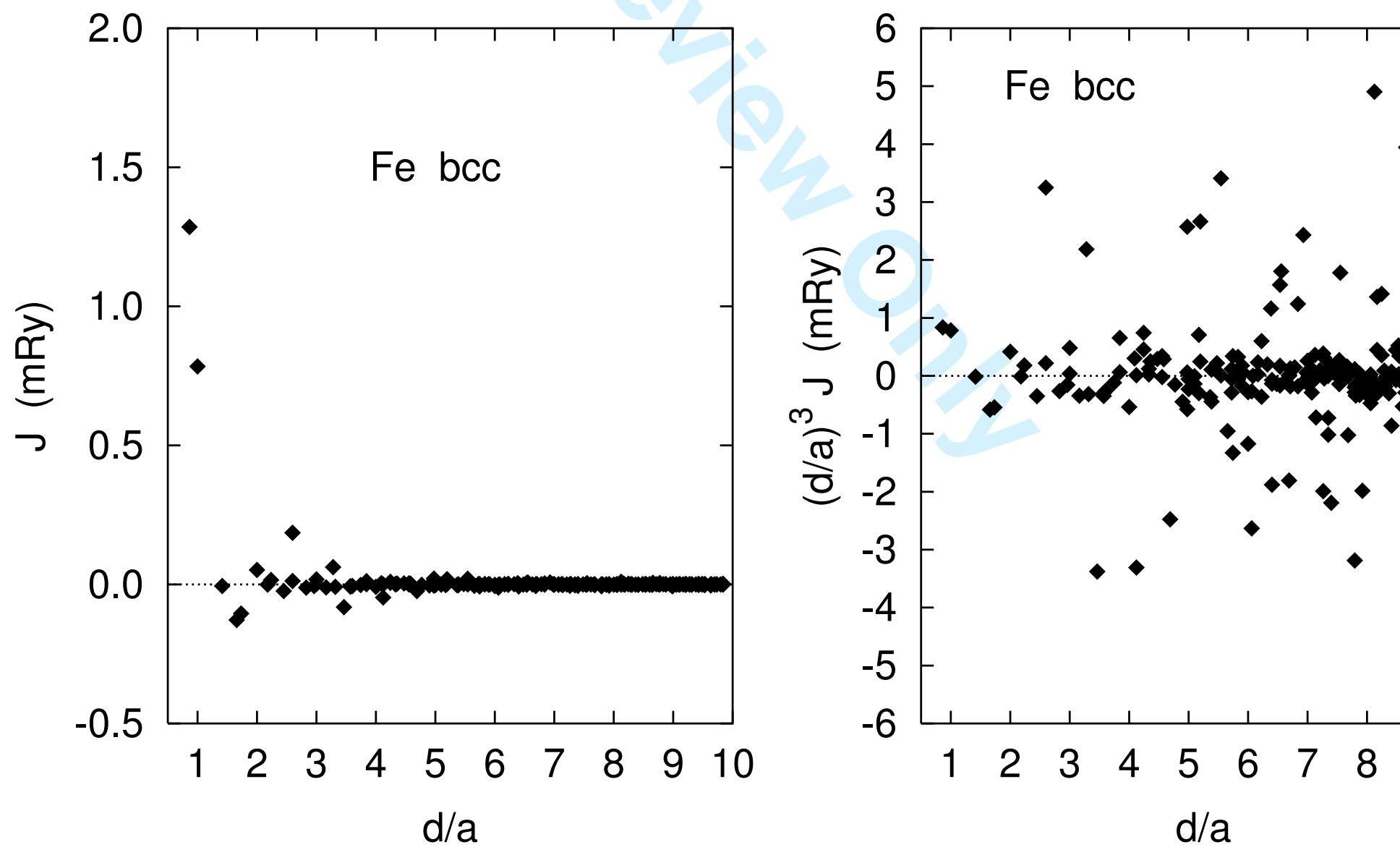

http://mc.manuscriptcentral.com/pm-pml 


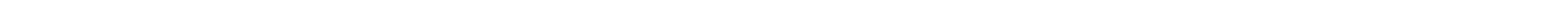



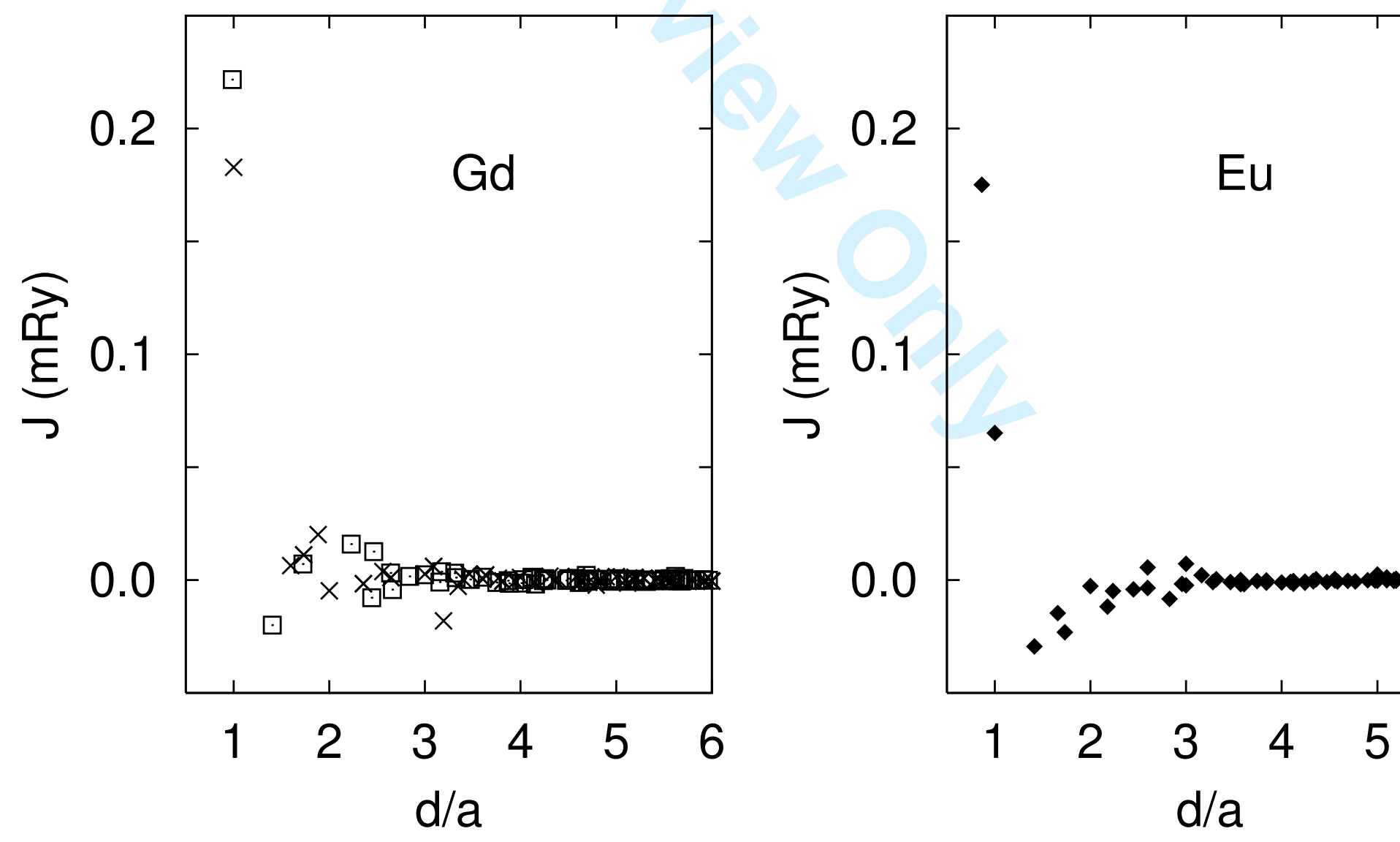

http://mc.manuscriptcentral.com/pm-pml 


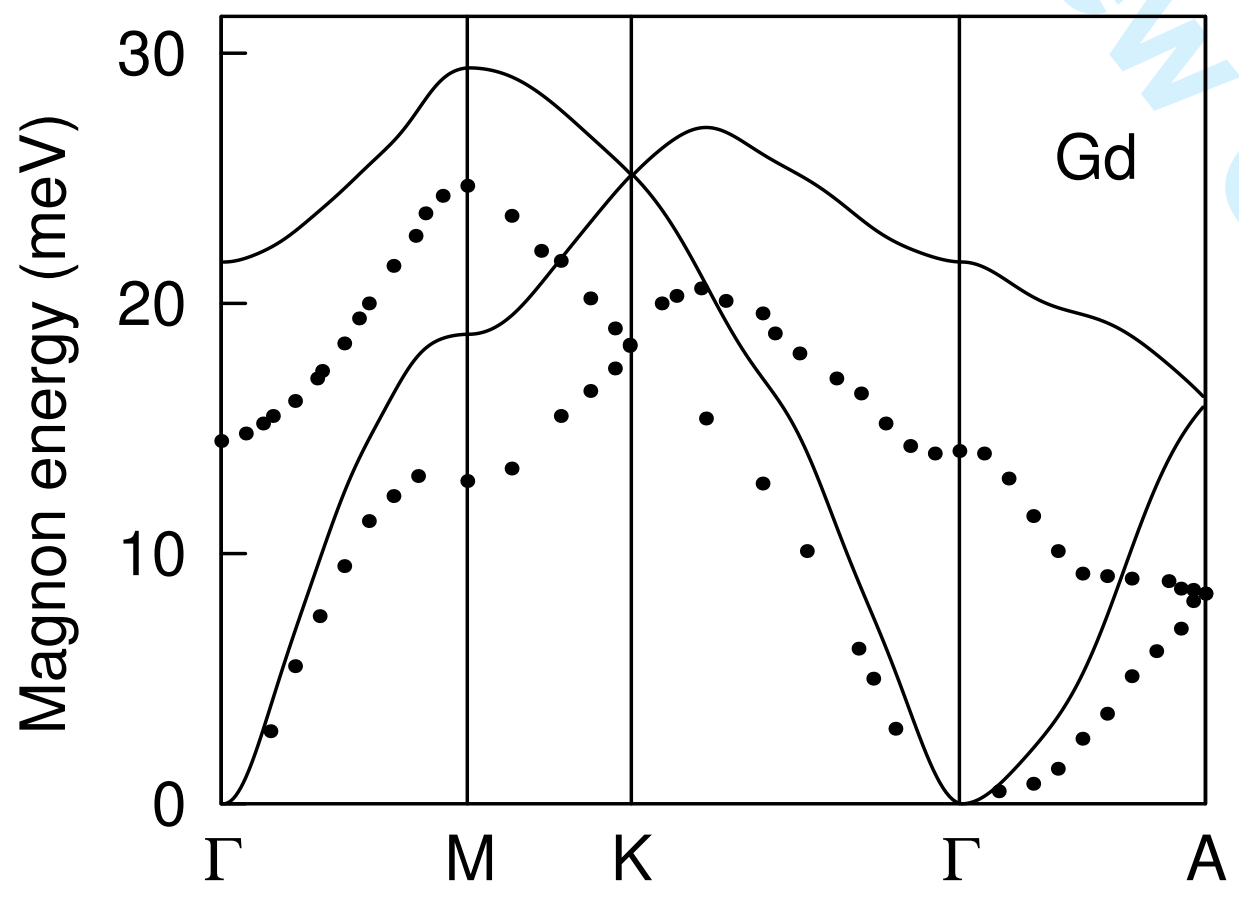




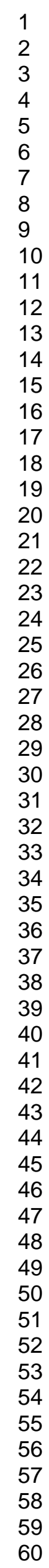

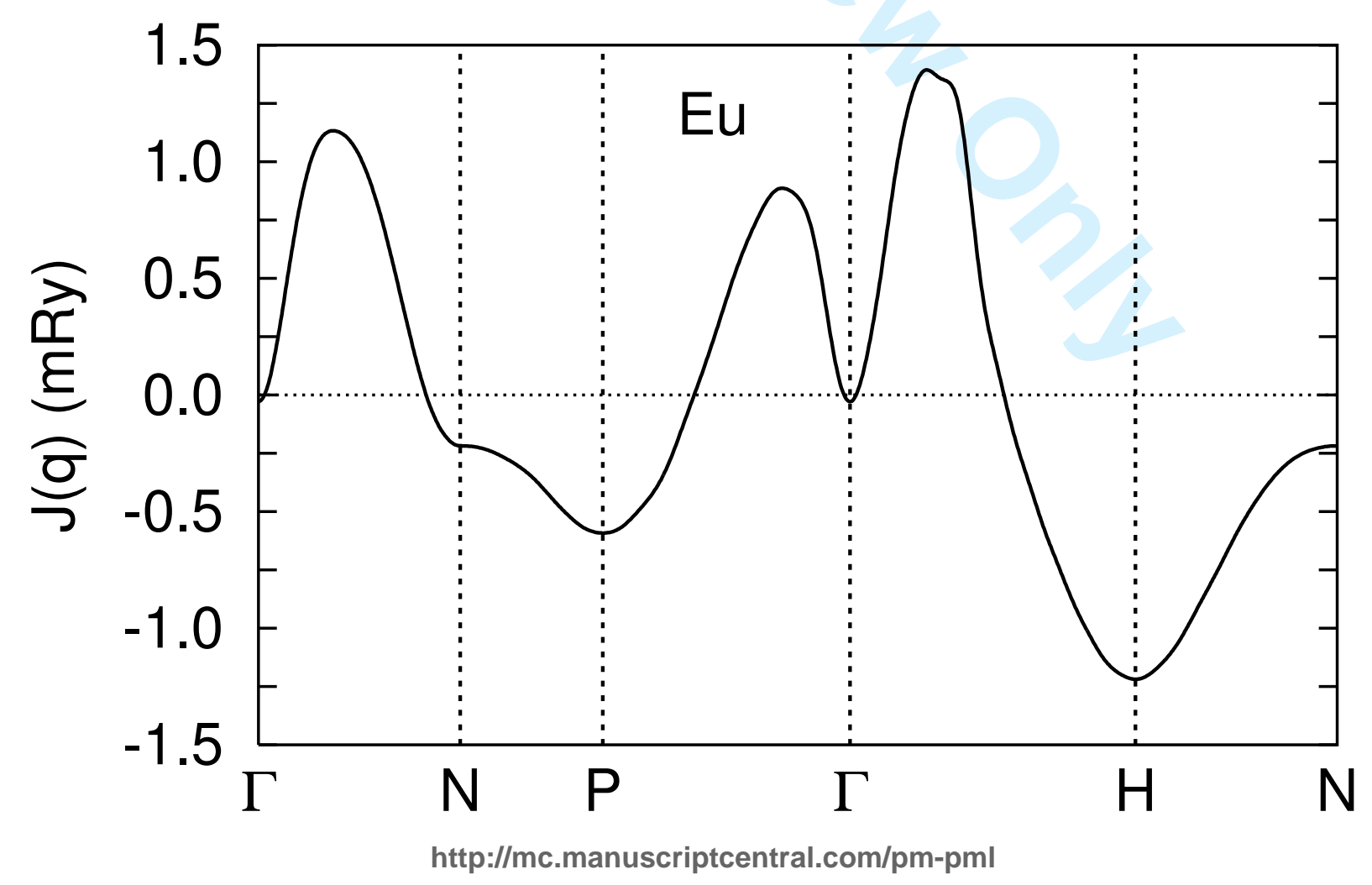




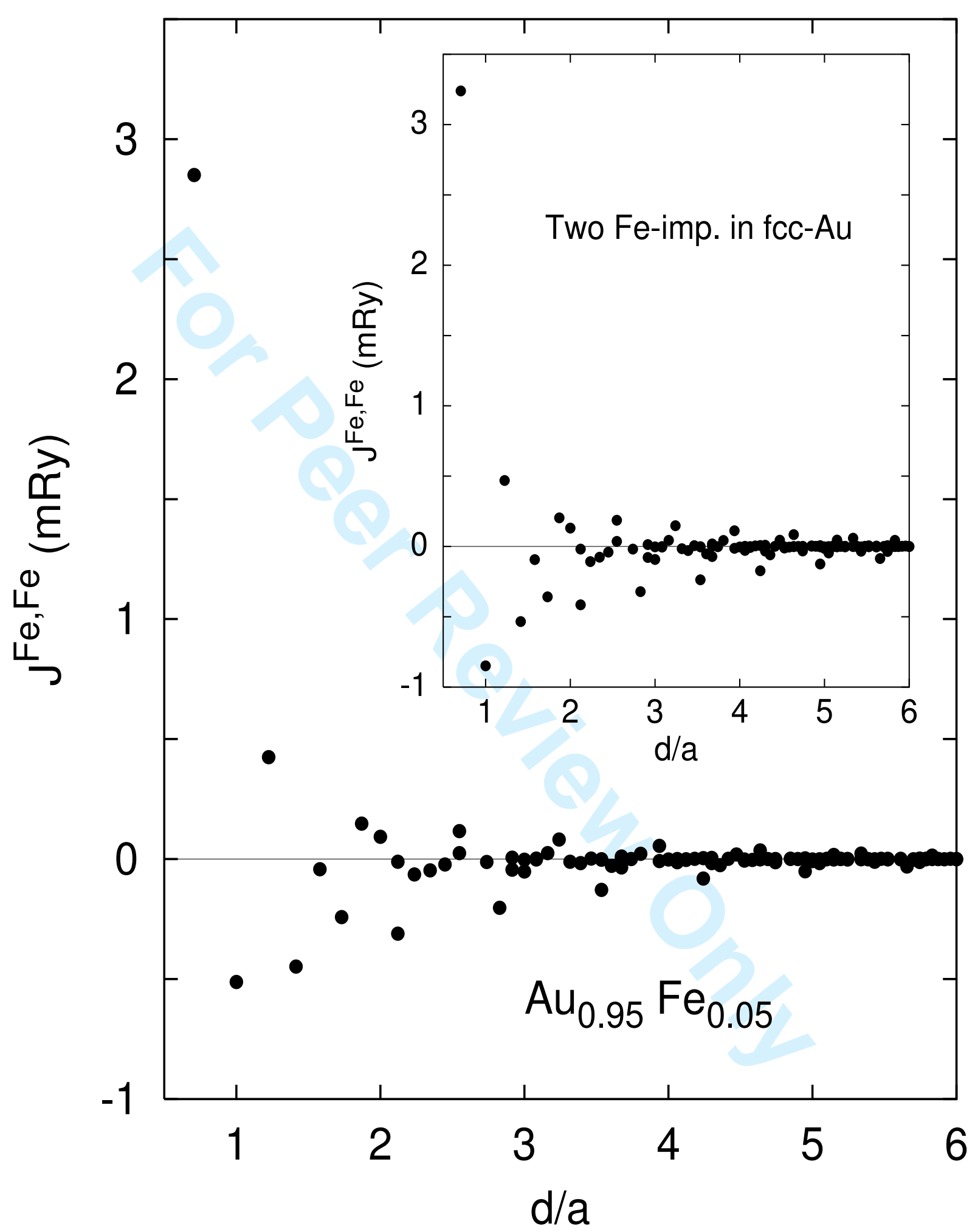

http://mc.manuscriptcentral.com/pm-pml 

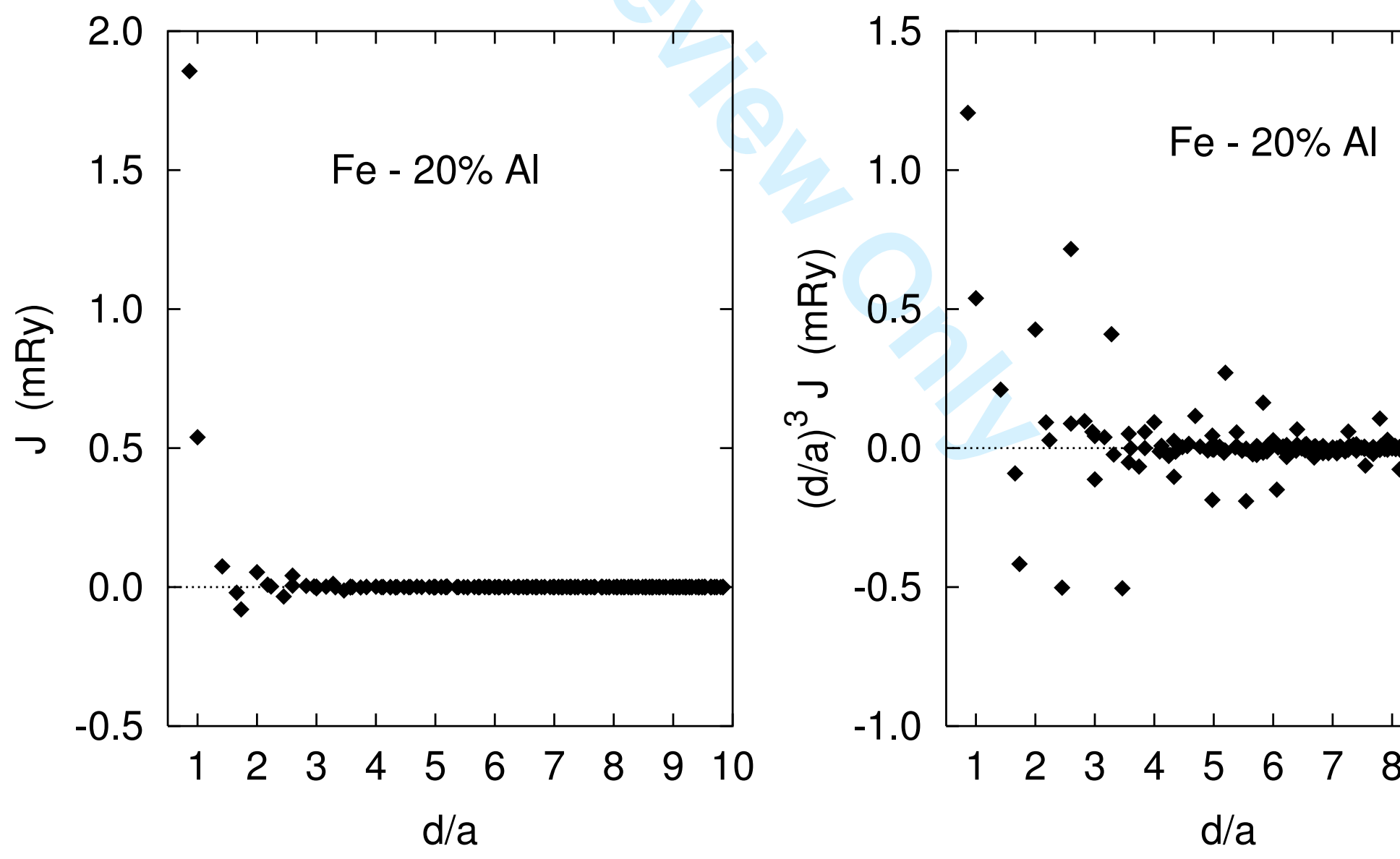

http://mc.manuscriptcentral.com/pm-pml 

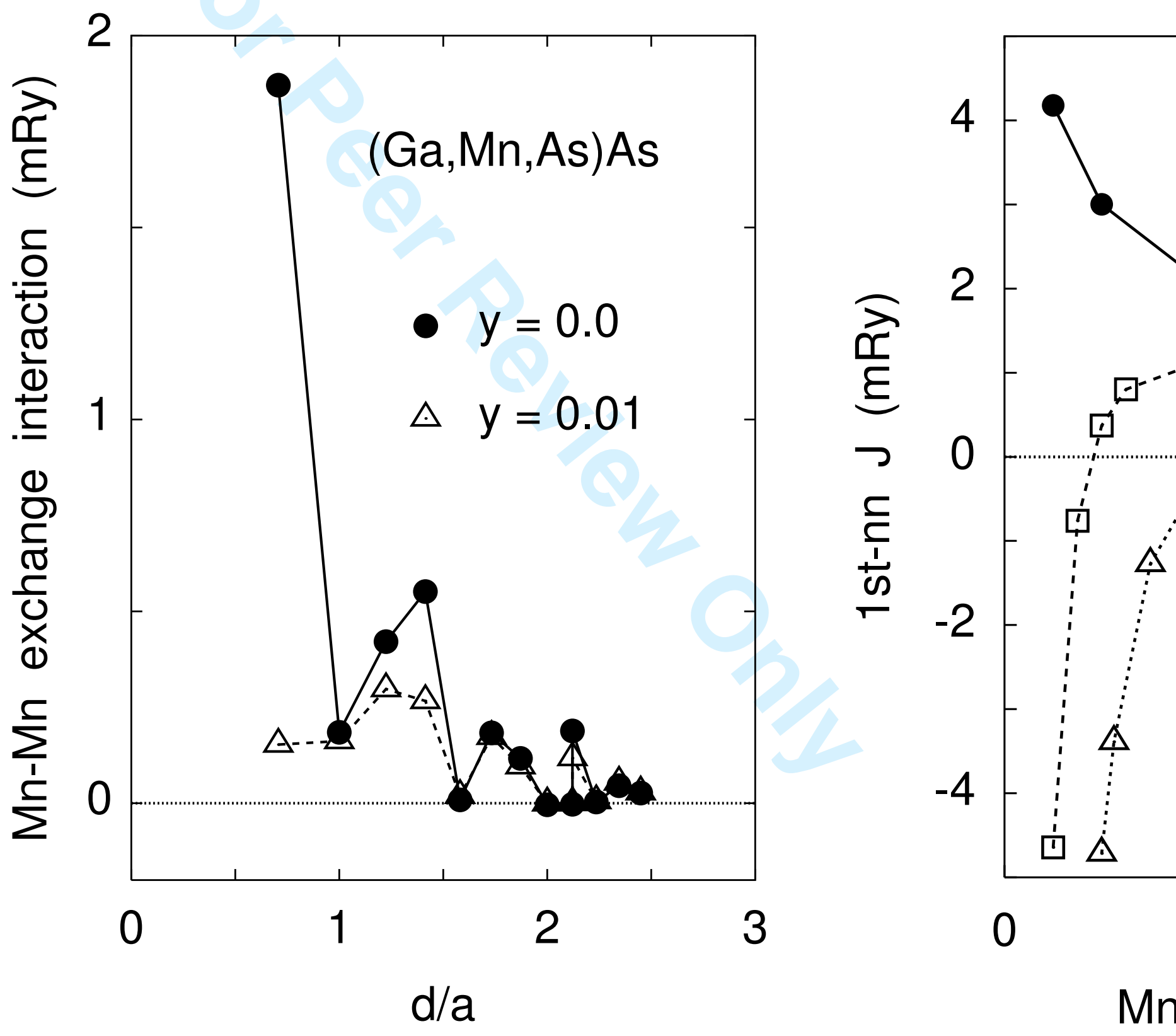


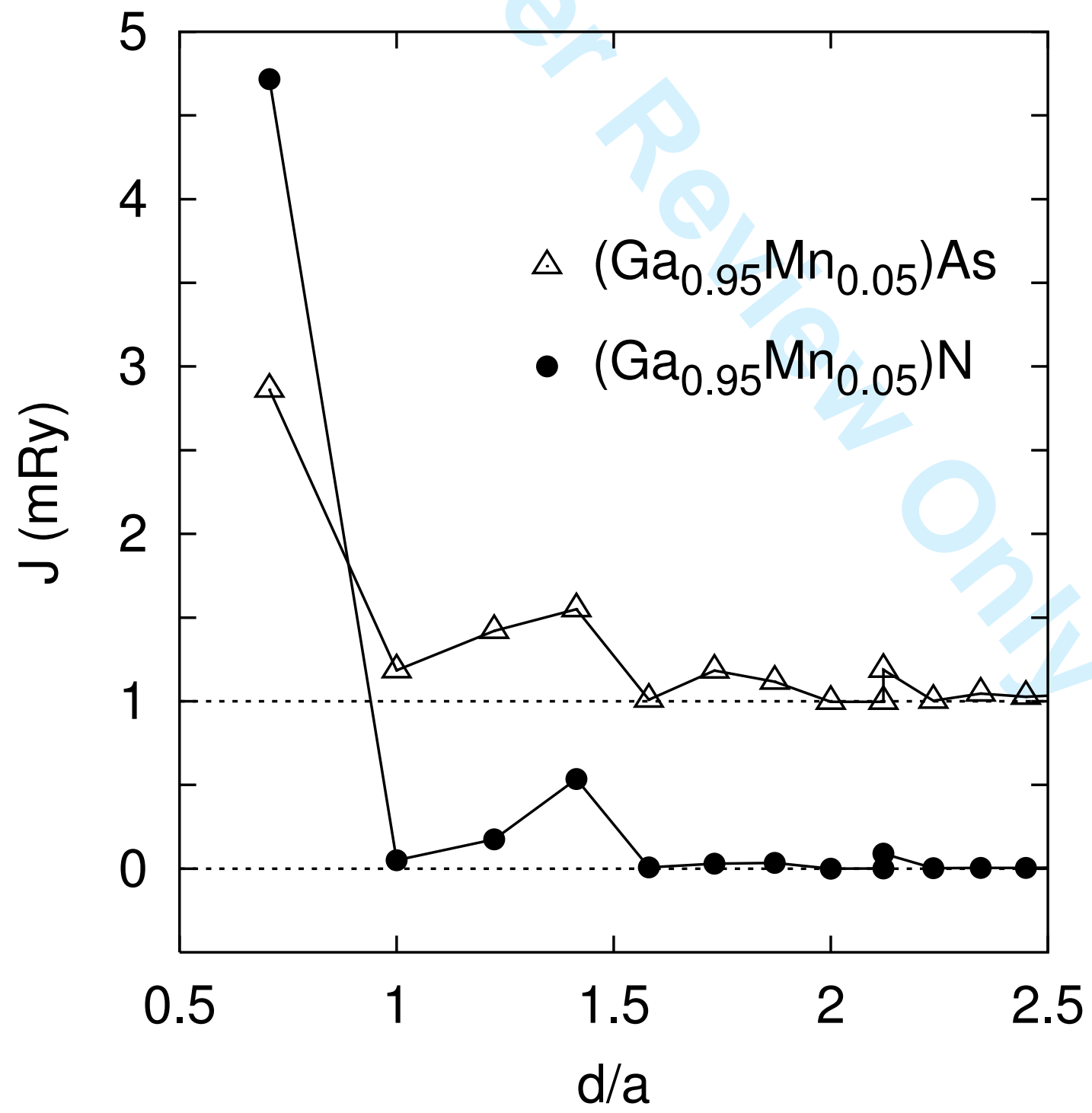

http://mc.manuscriptcentral.com/pm-pml 

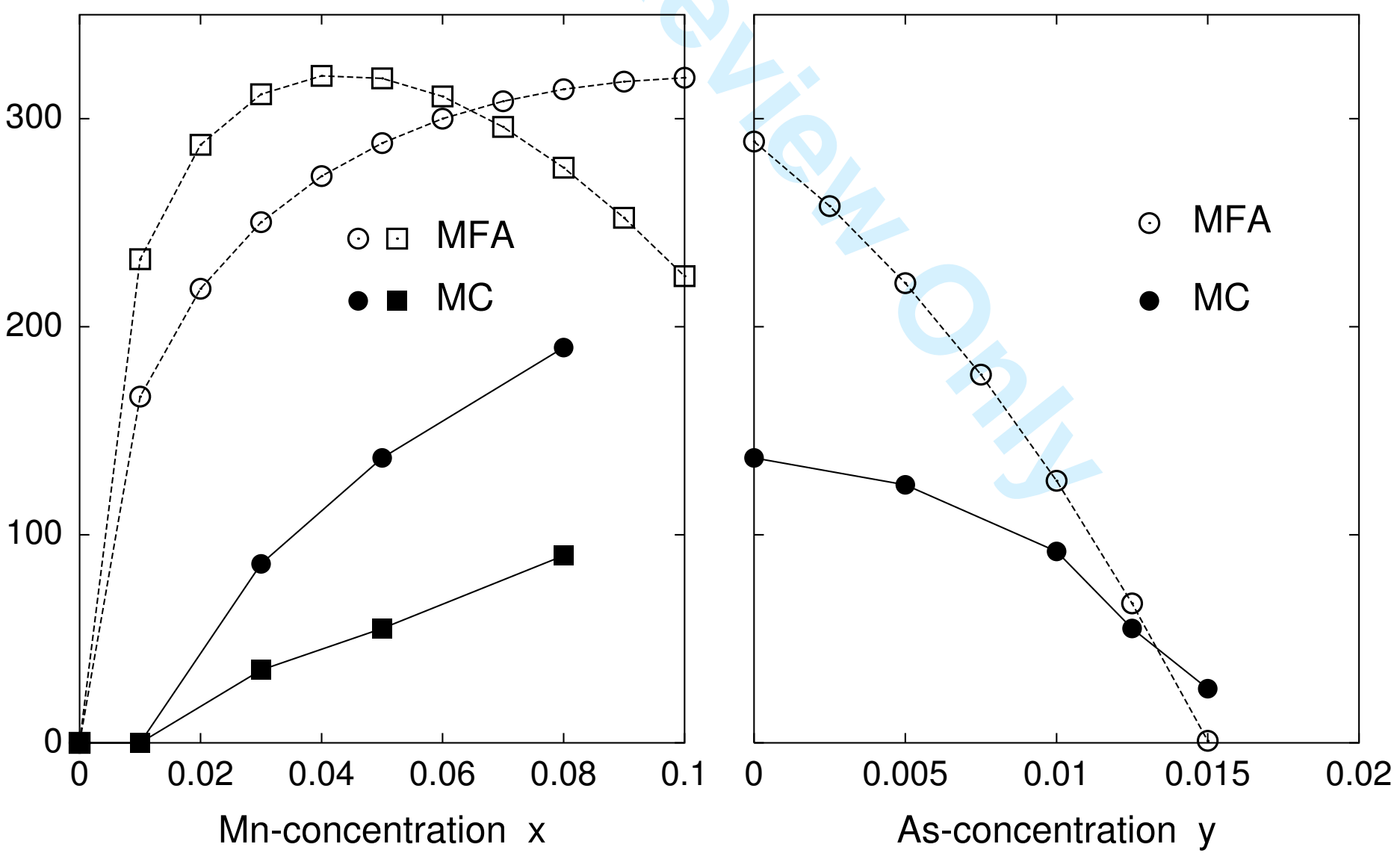


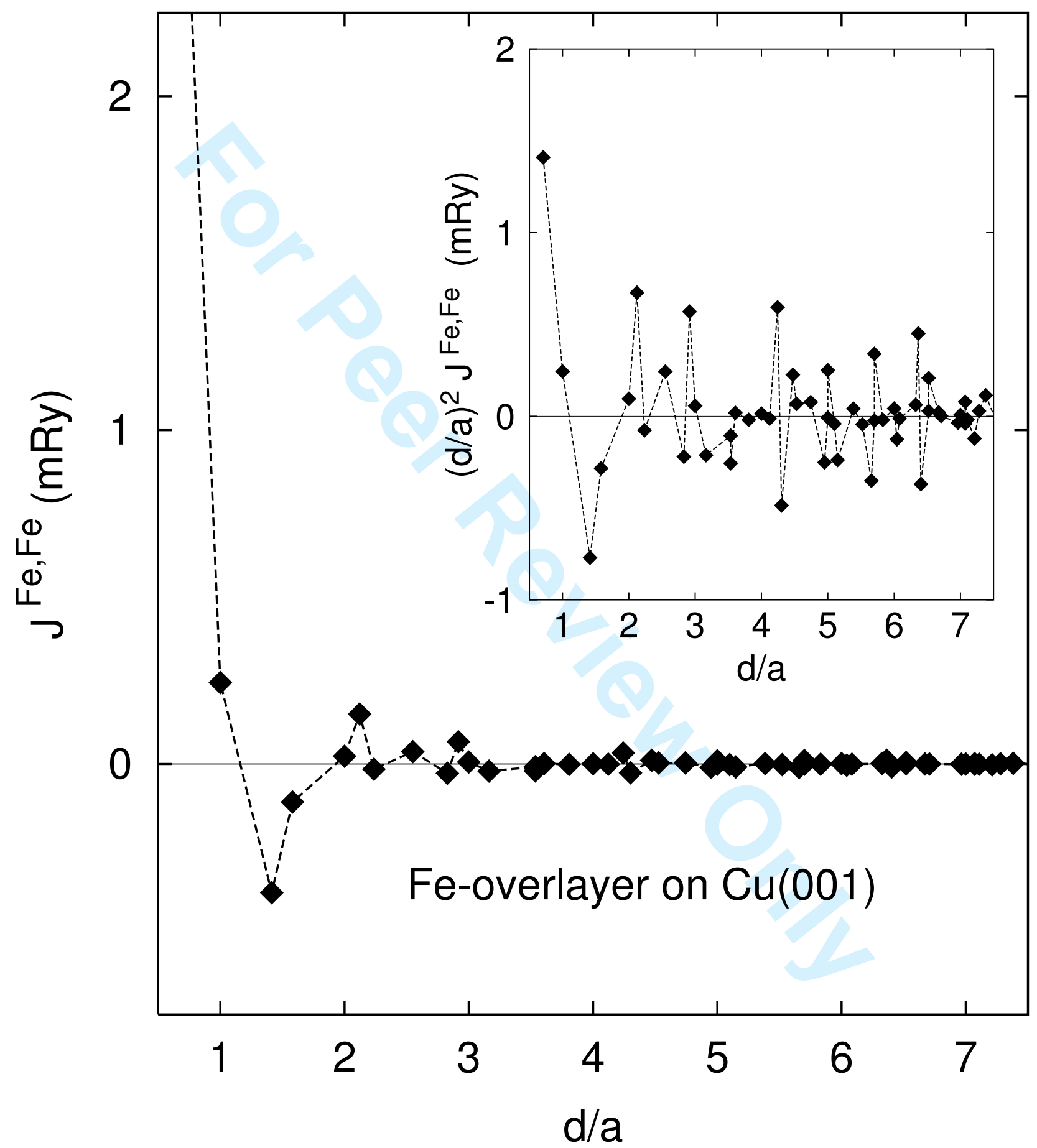

http://mc.manuscriptcentral.com/pm-pml 


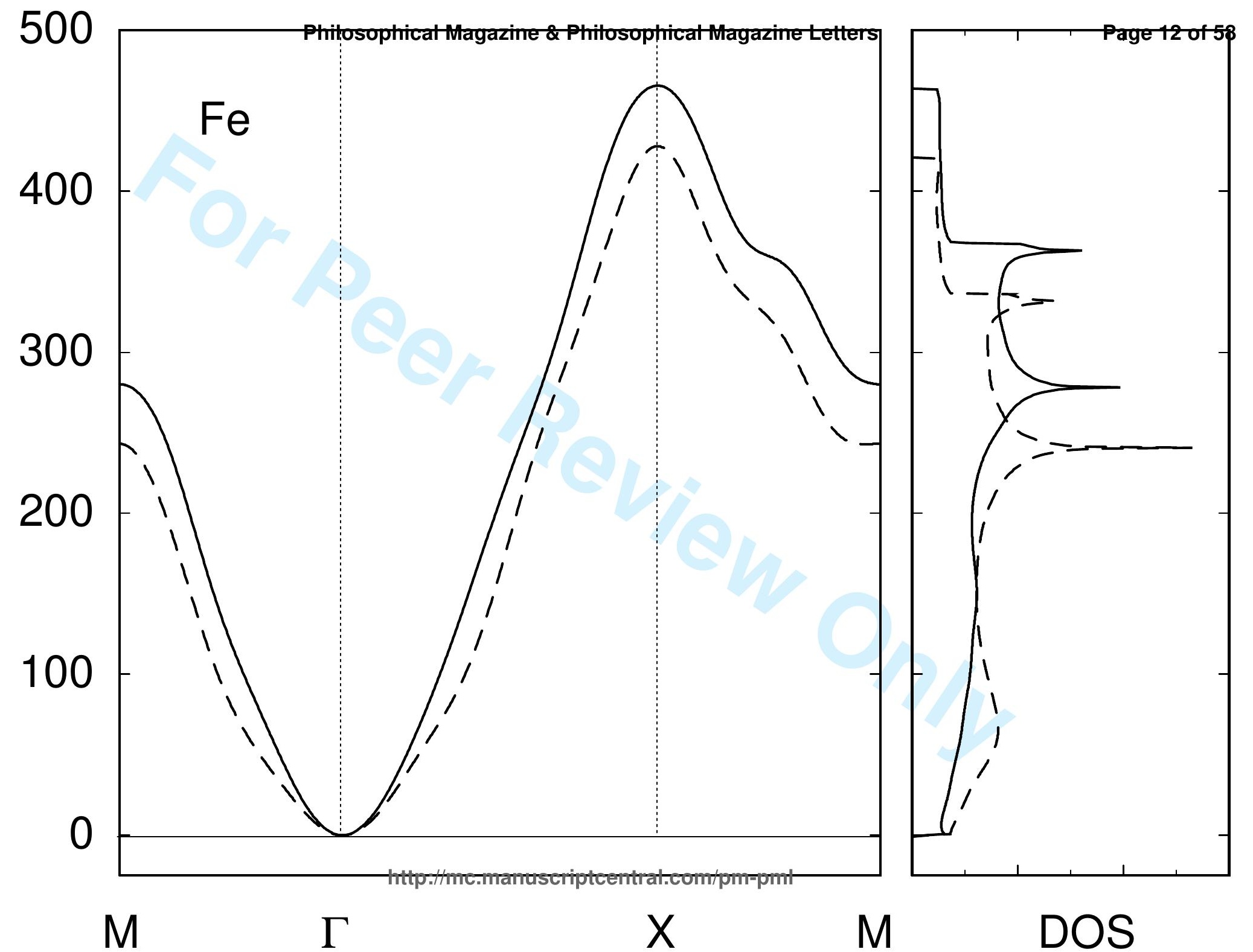




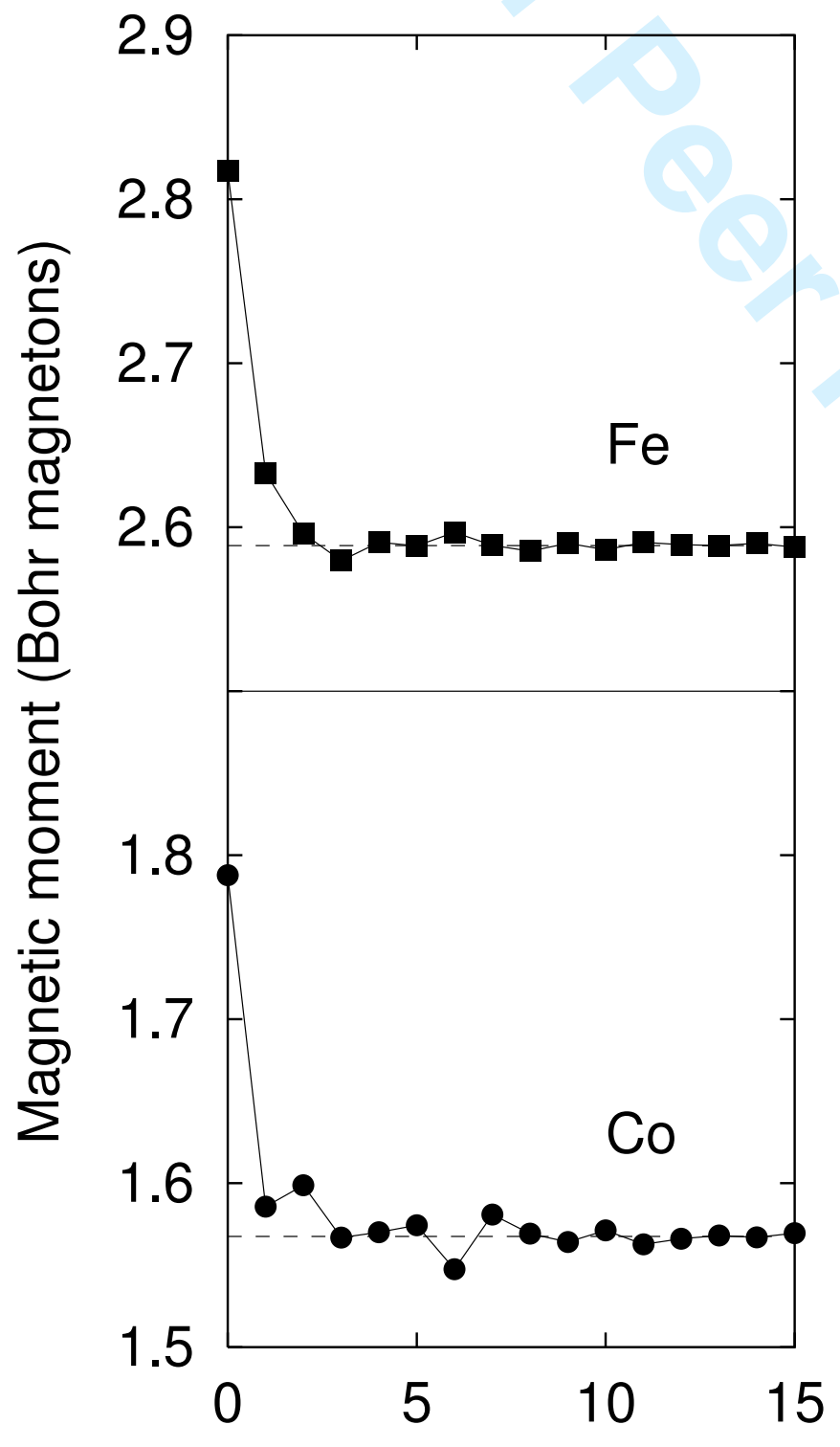

Cap-layer thickness (MLs)

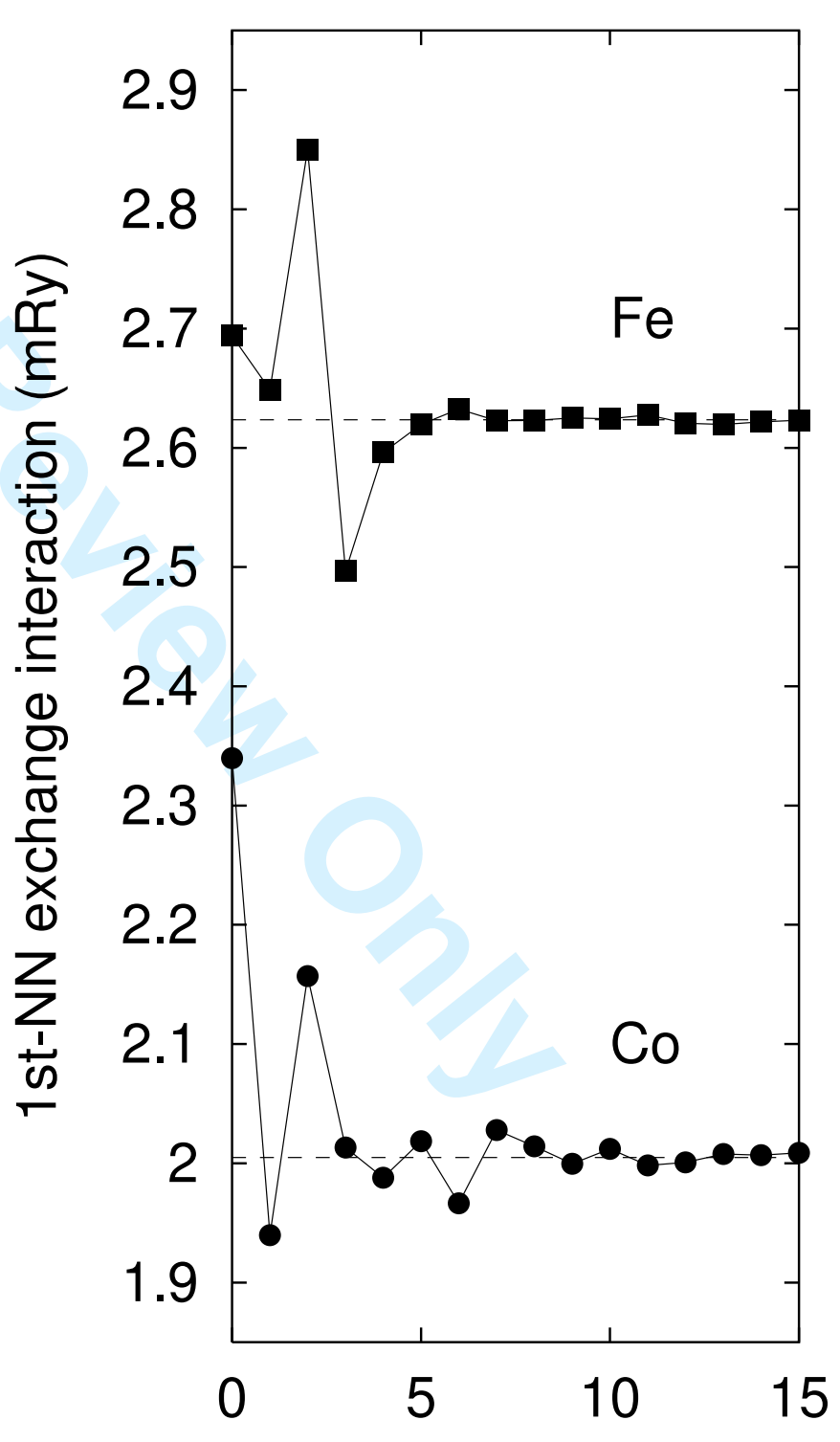

Cap-layer thickness (MLs) 


3

31

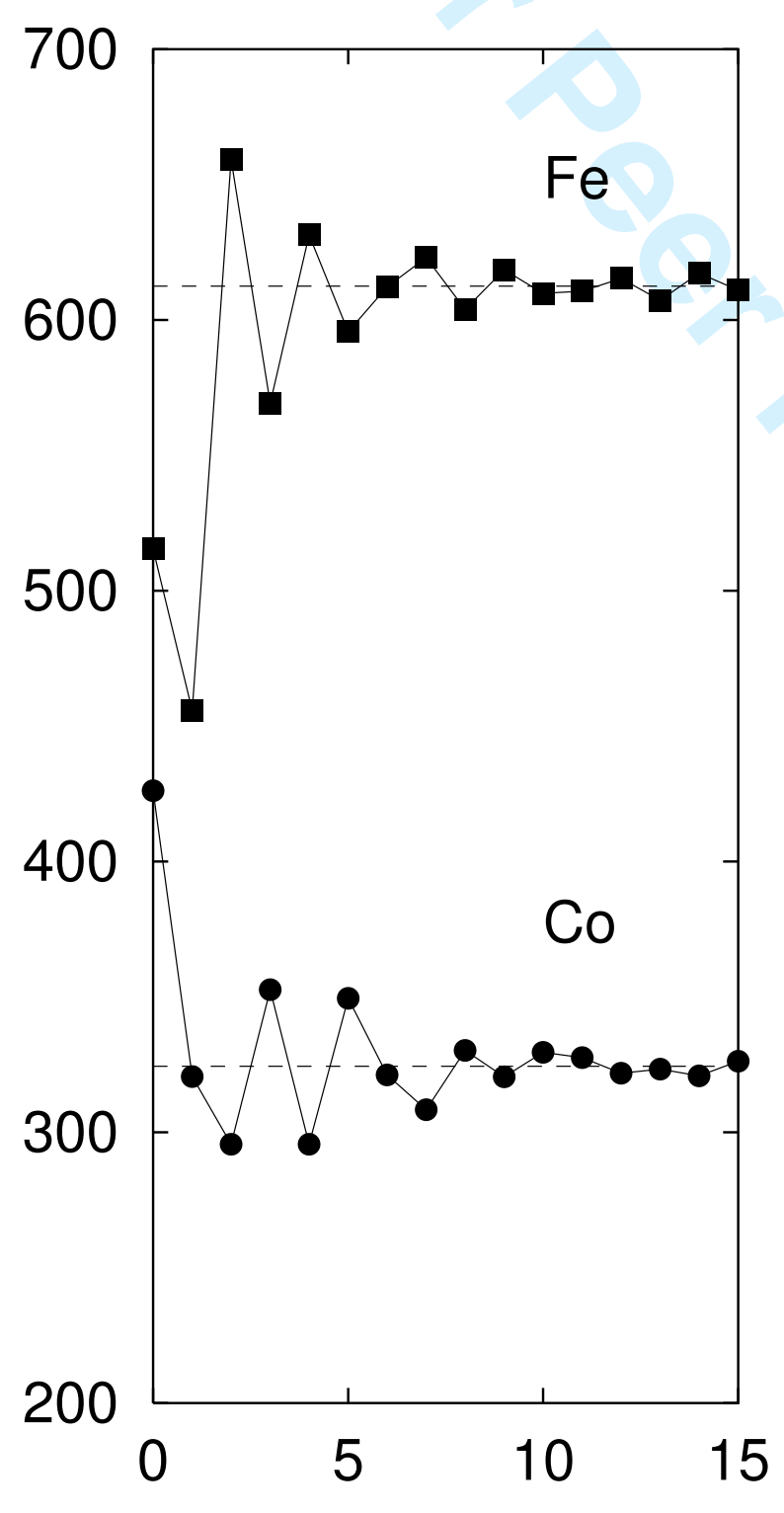

Cap-layer thickness (MLs)

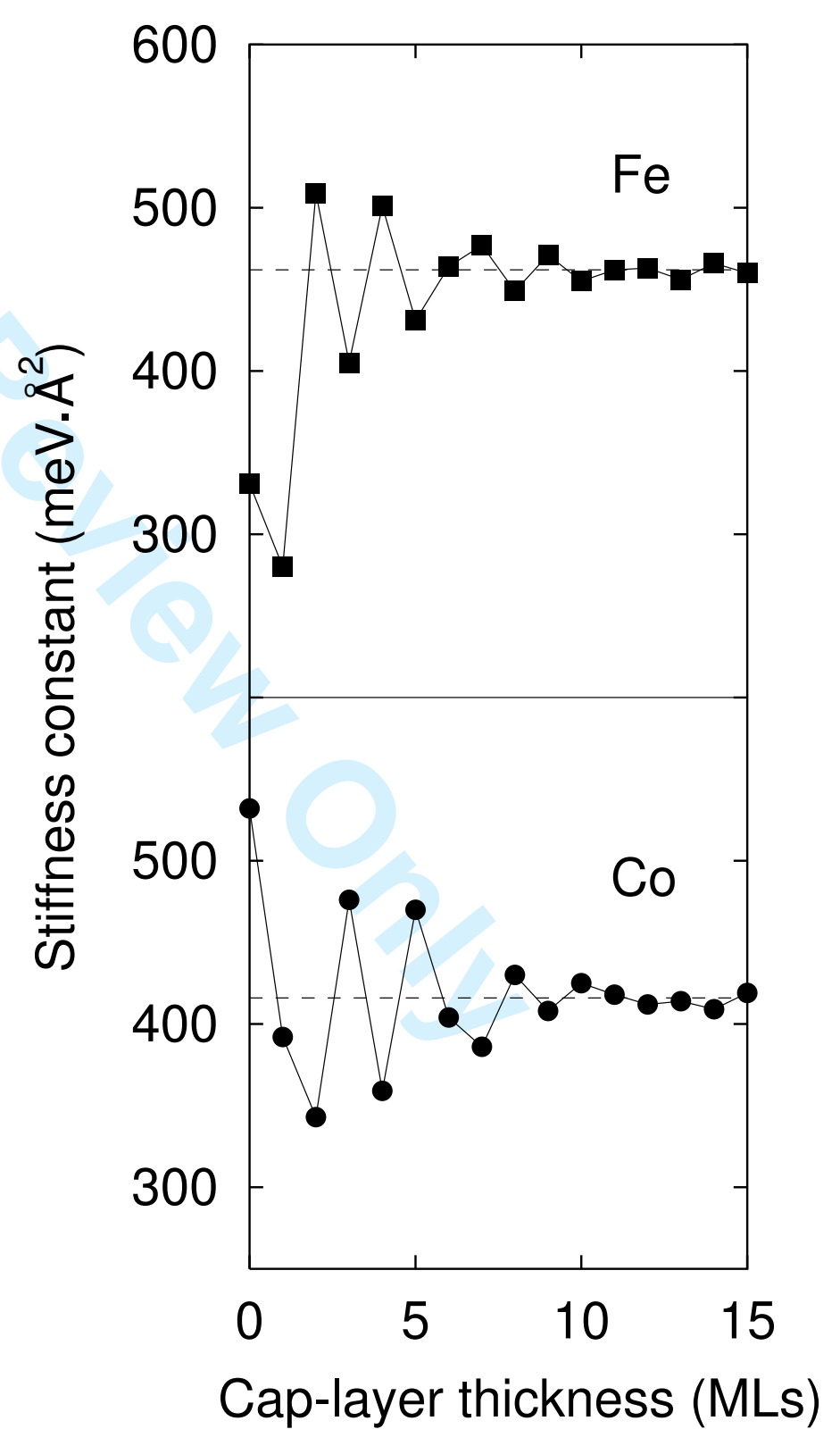



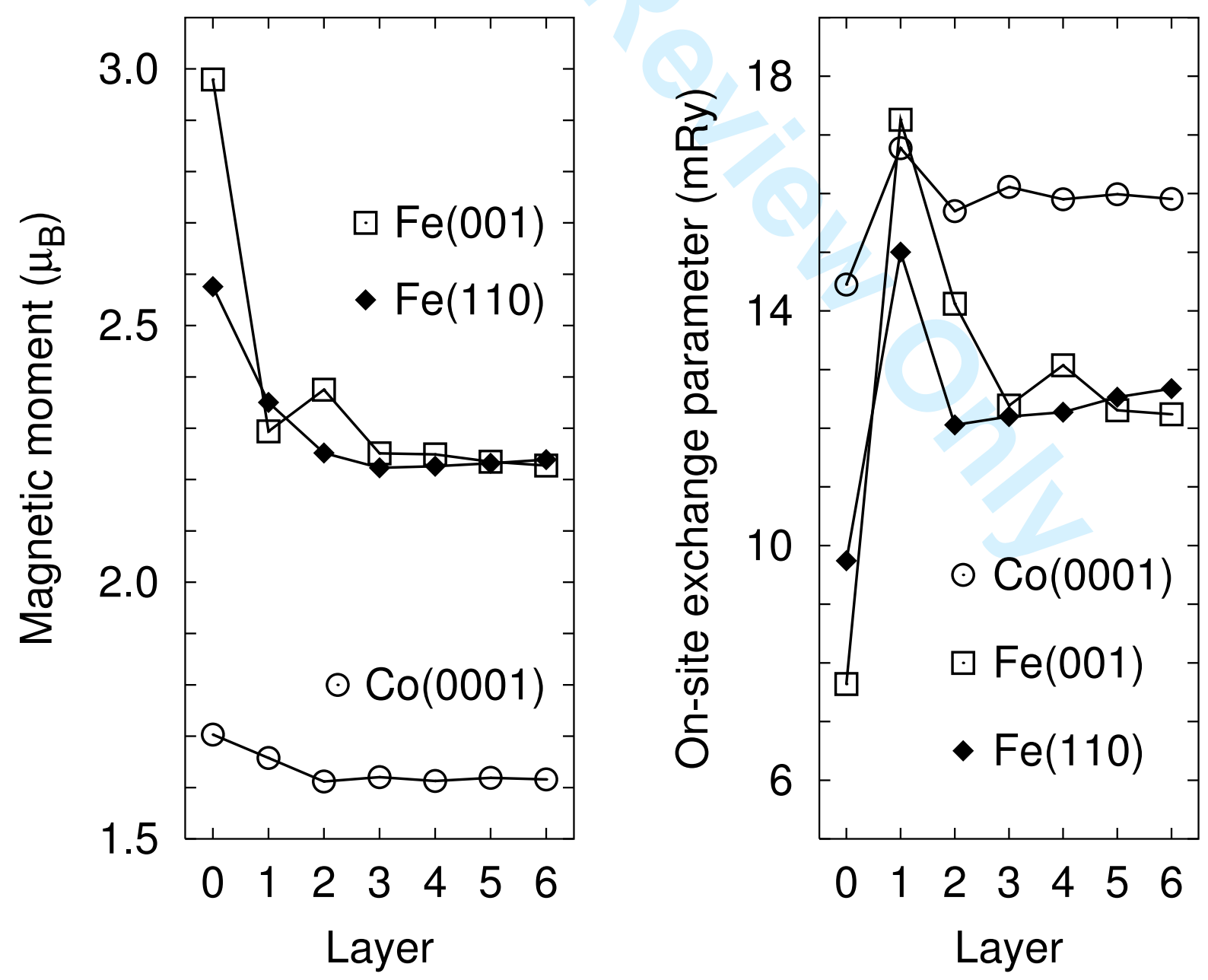

http://mc.manuscriptcentral.com/pm-pml 

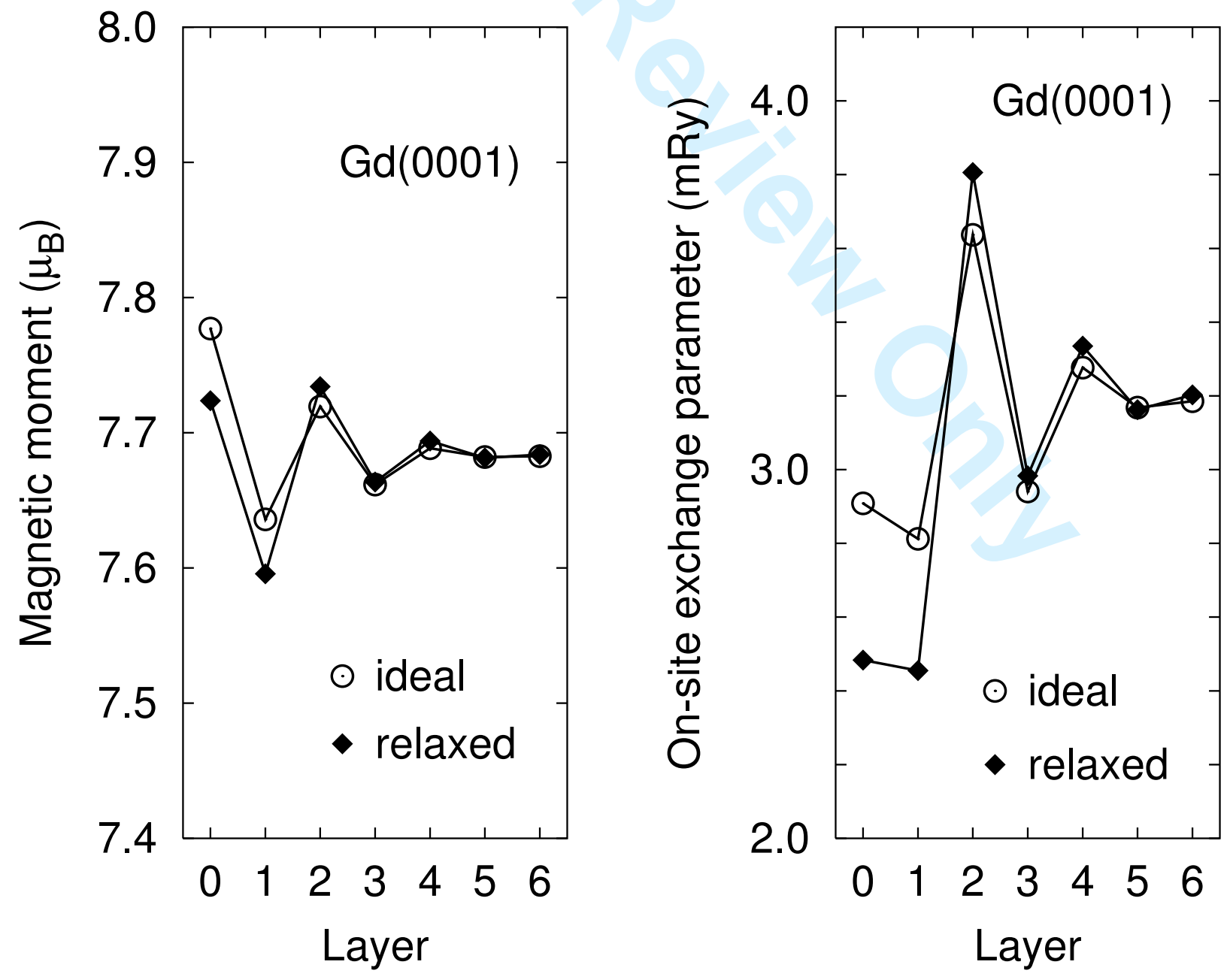

http://mc.manuscriptcentral.com/pm-pml 


\title{
Exchange interactions, spin waves, and transition temperatures in itinerant magnets
}

\author{
I. Turek, ${ }^{1,2,3, *}$ J. Kudrnovský, ${ }^{3,4,5}$ V Drchal, ${ }^{3,4}$ and P Bruno ${ }^{5}$
}

October 14, 2005

\author{
${ }^{1}$ Institute of Physics of Materials, Academy of Sciences of the Czech Republic, Žižkova 22, \\ CZ-61662 Brno, The Czech Republic \\ ${ }^{2}$ Department of Electronic Structures, Faculty of Mathematics and Physics, Charles Uni- \\ versity, Ke Karlovu 5, CZ-12116 Prague 2, The Czech Republic \\ ${ }^{3}$ Center for Computational Materials Science, Technical University of Vienna, Getreide- \\ markt 9, A-1060 Vienna, Austria \\ ${ }^{4}$ Institute of Physics, Academy of Sciences of the Czech Republic, Na Slovance 2, CZ- \\ 18221 Prague 8, The Czech Republic \\ ${ }^{5}$ Max-Planck-Institut für Mikrostrukturphysik, Weinberg 2, D-06120 Halle, Germany \\ * corresponding author,e-mail: turek@ipm.cz
}

\begin{abstract}
The contribution reviews an ab initio two-step procedure to determine exchange interactions, spin-wave spectra, and thermodynamic properties of itinerant magnets. In the first step, the selfconsistent electronic structure of a system is calculated for a collinear spin structure at zero temperature. In the second step, parameters of an effective classical Heisenberg Hamiltonian are determined using the magnetic force theorem and the one-electron Green functions. The Heisenberg Hamiltonian and methods of statistical physics are employed in subsequent evaluation of magnon dispersion laws, spin-wave stiffness constants, and Curie/Néel temperatures. Applicability of the developed scheme is illustrated by selected properties of various systems such as transition and rare-earth metals, disordered alloys including diluted magnetic semiconductors, ultrathin films, and surfaces. A comparison to other $a b$ initio approaches is presented as well.
\end{abstract}

PACS numbers: 75.10.Hk, 71.15.Mb 


\section{Introduction Phical Magazine \& Philosophical Magazine Letters}

The quantitative description of ground-state and finite-temperature properties of metallic systems represents a long-term challenge for solid state theory. Practical implementation of density functional theory (DFT) $[1,2,3]$ led to excellent parameter-free description of ground-state properties of metallic magnets, including traditional bulk metals and ordered alloys as well as systems without the perfect three-dimensional periodicity, such as, e.g., disordered alloys, surfaces and thin films. On the other hand, an accurate quantitative treatment of excited states and finite-temperature properties of these systems remains a challenge for $a b$ initio theory $[4,5,6,7]$ despite existing formal extensions of the DFT to time-dependent phenomena [8] and finite temperatures [9]. The usual local spin-density approximation (LSDA) [3] fails to capture important features of excited states, in particular the magnetic excitations responsible for the decrease of the magnetization with temperature and for the magnetic phase transition.

In developing a practical parameter-free scheme for the finite-temperature magnetism, one has to rely on additional assumptions and approximations the validity of which has to be chosen on the basis of physical arguments. The purpose of this contribution is to review theoretical backgrounds, numerical aspects, and selected results of an approach formulated nearly two decades ago [10, 11, 12] (see reference [13] for a recent review), and applied by the present authors to a number of qualitatively different systems $[14,15,16,17,18,19,20,21,22,23,24,25]$. The review is organized as follows: section 2 lists the underlying physical concepts and approximations of the scheme and section 3 deals with computational details and specific problems related to its numerical implementation. Examples of applications are given in section 4: bulk transition metals (section 4.1), rare-earth metals (section 4.2), substitutional impurities and disordered alloys (section 4.3), diluted magnetic semiconductors (section 4.4), two-dimensional ferromagnets (section 4.5), and surfaces of bulk ferromagnets (section 4.6). Comparisons to other authors using the same (or similar) approach are made throughout section 4, while a critical discussion of the scheme and a brief comparison to alternative approaches are left to the last section (section 5).

\section{Formalism}

It is well known that magnetic excitations in itinerant ferromagnets are basically of two different types, namely, the Stoner excitations, in which an electron is excited from an occupied state of the majority-spin band to an empty state of the minority-spin band and creates an electron-hole pair of triplet spin, and the spin waves, or magnons, which correspond to collective transverse fluctuations of the magnetization direction. Near the bottom of the excitation spectrum, the density of states of magnons is considerably larger than that of corresponding Stoner excitations (associated with longitudinal fluctuations of the magnetization), so that the thermodynamics in the low-temperature regime is completely dominated by magnons and Stoner excitations can be neglected. Therefore it seems reasonable to extend this approximation up to the Curie temperature and to derive an $a b$ initio technique of finite-temperature magnetism by neglecting systematically the Stoner excitations.

With thermodynamic properties in mind, we are primarily interested in the long- 

in which the precession of the magnetization due to a spin wave is neglected when calculating the associated change of electronic energy. The condition of validity of this approximation is that the precession time of the magnetization should be large as compared to characteristic times of electronic motion, i.e., the hopping time of an electron from a given site to a neighbouring one and the precession time of the spin of an electron subject to the exchange field. In other words, the spin-wave energies should be small as compared to the band width and to the exchange splitting. This approximation becomes exact in the limit of long wavelength magnons, so that the spin-wave stiffness constants calculated in this way are in principle exact.

This procedure corresponds to evaluation of changes of the total energy of a ferromagnet due to infinitesimal changes of the directions of its local magnetic moments associated with individual lattice sites $\mathbf{R}$. The directions of the moments are specified by unit vectors $\mathbf{e}_{\mathbf{R}}$. An exact calculation of the total energy $\mathcal{E}\left\{\mathbf{e}_{\mathbf{R}}\right\}$ of a prescribed spin configuration leads to the constrained DFT $[27,28]$, which allows to obtain the ground-state energy for a system subject to certain constraints. The latter are naturally incorporated into the DFT in terms of Lagrange multipliers. In the present case, the constraint consists in imposing a given configuration of spin-polarization directions, namely, the spin moment of the $\mathbf{R}$-th atomic (Wigner-Seitz) cell pointing along $\mathbf{e}_{\mathbf{R}}$. The Lagrange multipliers can be interpreted as magnetic fields $\mathbf{B}_{\mathbf{R}}^{\perp}$ constant inside the cells with directions perpendicular to the unit vectors $\mathbf{e}_{\mathbf{R}}$. Note that intracell non-collinearity of the spin-polarization is neglected since we are primarily interested in low-energy excitations due to intercell non-collinearity. In the so-called frozen-magnon approach, one chooses the constrained spin-polarization configuration to be the one of a spin wave with the wave vector $\mathbf{q}$ and computes the spin-wave energy $E(\mathbf{q})$ directly by employing the generalized Bloch theorem for a spin-spiral configuration [28, 29].

In a real-space approach, adopted here, one calculates directly the energy change associated with a constrained rotation of the spin-polarization axes in two cells $\mathbf{e}_{\mathbf{R}}$ and $\mathbf{e}_{\mathbf{R}^{\prime}}$. This represents a highly non-trivial task which requires selfconsistent electronic structure calculations for non-collinear spin-polarized systems without translational periodicity. We neglect relativistic effects (spin-orbit coupling, dipolar interaction) in the following. Restriction to infinitesimal changes of the moment directions, $\delta \mathbf{u}_{\mathbf{R}}=\mathbf{e}_{\mathbf{R}}-\mathbf{e}^{0}$, perpendicular to the direction of the ground-state magnetization $\mathbf{e}^{0}$, leads to an expansion of $\mathcal{E}\left\{\mathbf{e}_{\mathbf{R}}\right\}$ to second order in $\delta \mathbf{u}_{\mathbf{R}}$ of the form $[11,30]$

$$
\Delta \mathcal{E}\left\{\delta \mathbf{u}_{\mathbf{R}}\right\}=\sum_{\mathbf{R R}^{\prime}} A_{\mathbf{R R}^{\prime}} \delta \mathbf{u}_{\mathbf{R}} \cdot \delta \mathbf{u}_{\mathbf{R}^{\prime}} .
$$

This expression can be extended to finite changes of the moment directions using an effective Heisenberg Hamiltonian (EHH)

$$
H_{\mathrm{eff}}\left\{\mathbf{e}_{\mathbf{R}}\right\}=-\sum_{\mathbf{R} \mathbf{R}^{\prime}} J_{\mathbf{R R}^{\prime}} \mathbf{e}_{\mathbf{R}} \cdot \mathbf{e}_{\mathbf{R}^{\prime}} .
$$

The constants $J_{\mathbf{R R}^{\prime}}$ in (2), the pair exchange interactions, are parameters of the EHH which satisfy $J_{\mathbf{R R}^{\prime}}=J_{\mathbf{R}^{\prime} \mathbf{R}}$ and $J_{\mathbf{R R}}=0$. They are related to the coupling constants $A_{\mathbf{R R}^{\prime}}$ of (1) by

$$
A_{\mathbf{R R}^{\prime}}=-J_{\mathbf{R R}^{\prime}}+\delta_{\mathbf{R R}^{\prime}}\left(\sum_{\mathbf{R}^{\prime \prime}} J_{\mathbf{R}^{\prime \prime} \mathbf{R}}\right)
$$


so that an important sum rule

$$
\sum_{\mathbf{R}} A_{\mathbf{R R}^{\prime}}=\sum_{\mathbf{R}^{\prime}} A_{\mathbf{R R}^{\prime}}=0
$$

is satisfied which guarantees that the total energy remains invariant upon a uniform rotation of the magnetization.

The practical calculations of the exchange interactions $J_{\mathbf{R R}^{\prime}}$ in ferromagnets are greatly simplified by employing the magnetic force theorem $[10,11]$ (a similar approach was also suggested for magnetic impurities in a non-magnetic host [31]). The infinitesimal changes of the total energy (1) can be expressed using changes in one-particle eigenvalues due to non-selfconsistent changes of the effective one-electron potential accompanying the infinitesimal rotations of spin quantization axes, i.e., without any additional selfconsistent calculations besides that for the collinear ground state. The resulting pair exchange interactions are given by $[11,30]$

$$
\begin{aligned}
& J_{\mathbf{R R}^{\prime}}=\frac{1}{\pi} \operatorname{Im} \int_{-\infty}^{E_{\mathrm{F}}} d E \int_{\Omega_{\mathbf{R}}} d \mathbf{r} \int_{\Omega_{\mathbf{R}^{\prime}}} d \mathbf{r}^{\prime} B_{\mathrm{xc}}(\mathbf{r}) G^{\uparrow}\left(\mathbf{r}, \mathbf{r}^{\prime} ; E^{+}\right) \\
& \times B_{\mathrm{xc}}\left(\mathbf{r}^{\prime}\right) G^{\downarrow}\left(\mathbf{r}^{\prime}, \mathbf{r} ; E^{+}\right),
\end{aligned}
$$

where $E_{\mathrm{F}}$ denotes the Fermi level, $\Omega_{\mathbf{R}}$ denotes the $\mathbf{R}$-th atomic cell, $B_{\mathrm{xc}}(\mathbf{r})$ is the exchangecorrelation magnetic field, $2 B_{\mathrm{xc}}(\mathbf{r})=V^{\downarrow}(\mathbf{r})-V^{\uparrow}(\mathbf{r})$, where $V^{\sigma}(\mathbf{r})$ is the selfconsistent LSDA potential, $\sigma$ is the spin index $(\sigma=\uparrow, \downarrow), E^{+}=E+\mathrm{i} 0$, and $G^{\sigma}\left(\mathbf{r}, \mathbf{r}^{\prime} ; E^{+}\right)$is the one-electron retarded Green function for the same potential. It should be noted that the parameters $J_{\mathbf{R R}^{\prime}}$ determined by (5) do not contain contributions due to constraining magnetic fields necessary to keep a frozen non-collinear spin structure a stationary state of the Kohn-Sham equation. It can be expected that these contributions can be neglected in systems with large local magnetic moments. Validity of this approximation have been put on a more quantitative level in recent studies [30, 32, 33] (see also section 5).

Once the exchange parameters $J_{\mathbf{R R}^{\prime}}$ are obtained, the adiabatic spin-dynamics [34, $35,36,37,38$ ] can be easily determined from the EHH (2). One obtains the result known from spin-wave theories of localized ferromagnets: for ferromagnetic (FM) crystals with one atom in the primitive cell (Bravais lattice), the energy $E(\mathbf{q})$ of a zero-temperature magnon is related to the lattice Fourier transform $J(\mathbf{q})$ of the exchange interactions $J_{\mathbf{R R}^{\prime}}$ by

$$
E(\mathbf{q})=\frac{4}{M}[J(\mathbf{0})-J(\mathbf{q})], \quad J(\mathbf{q})=\sum_{\mathbf{R}} J_{\mathbf{0 R}} \exp (\mathbf{i q} \cdot \mathbf{R}),
$$

where $\mathbf{q}$ denotes a vector in the Brillouin zone (BZ) of the lattice and $M$ denotes the local moment magnitude in units of $\mu_{\mathrm{B}}$ ( $\mu_{\mathrm{B}}$ is the Bohr magneton). For cubic systems and for small $\mathbf{q}$-vectors, $E(\mathbf{q}) \approx D|\mathbf{q}|^{2}$ with the spin-wave stiffness constant equal to

$$
D=\frac{2}{3 M} \sum_{\mathbf{R}}|\mathbf{R}|^{2} J_{\mathbf{0 R}} .
$$

Finally, to obtain thermodynamic quantities such as the Curie temperature, methods of statistical mechanics have to be applied to the EHH (2). The simplest estimate of the Curie temperature $T_{\mathrm{C}}$ is based on a mean-field approximation (MFA) which leads to

$$
k_{\mathrm{B}} T_{\mathrm{C}}^{\mathrm{MFA}}=\frac{2}{3} J^{0}, \quad J^{0}=\sum_{\mathbf{R}} J_{\mathbf{0 R}}=J(\mathbf{0}),
$$


where $k_{\mathrm{B}}$ is the Boltzmann constant. The quantity $J^{0}$ denotes an on-site exchange parameter that reflects the molecular field experienced by a single moment in the ferromagnet. The limitations of the MFA are well known: it is correct only in the limit of high temperatures (above $T_{\mathrm{C}}$ ) and it fails to describe the low-temperature collective excitations (spin waves). An improved description of finite-temperature properties is provided by the Green function method within the random phase approximation (RPA) [39, 40]. The RPA is valid not only for high temperatures, but also at low temperatures, and it describes correctly the spin waves. In the intermediate regime (around $T_{\mathrm{C}}$ ), it represents a rather good approximation which may be viewed as an interpolation between the high and low temperature regimes. The RPA formula for the Curie temperature is given by

$$
\left(k_{\mathrm{B}} T_{\mathrm{C}}^{\mathrm{RPA}}\right)^{-1}=\frac{3}{2} \frac{1}{N} \sum_{\mathbf{q}}\left[J^{0}-J(\mathbf{q})\right]^{-1},
$$

where $N$ denotes the number of $\mathbf{q}$-vectors used in the BZ-average. It can be shown that $T_{\mathrm{C}}^{\mathrm{RPA}}$ is always smaller than $T_{\mathrm{C}}^{\mathrm{MFA}}$. It should be noted, however, that both the MFA and the RPA fail to describe correctly the critical behaviour and yield in particular incorrect critical exponents. Generalizations of the above relations (8) and (9) to systems with several inequivalent sublattices can be found, e.g., in references [41, 42]. Finally, the Curie temperature can also be estimated purely numerically by employing the method of Monte Carlo simulations applied to the EHH. This approach is in principle exact but its application to real itinerant systems requires inclusion of a sufficient number of neighbouring shells due to long-ranged interactions $J_{\mathbf{R R}^{\prime}}$ (see section 3.2).

It is worthwile to mention that, according to the Fröhlich-Simon-Spencer theorem [43], the classical Heisenberg model in dimension $d \geq 3$ has a transition to a FM state for the finite critical temperature $T_{\mathrm{C}}>0$. It is interesting that a formula for $T_{\mathrm{C}}$ which follows from the proof is identical with the RPA prescription (9). This means that the RPA yields a lower bound for $T_{\mathrm{C}}$. The original proof is valid for nearest-neighbour interactions, but it can be generalized [44] to a model with finite range of interactions, provided that all the interactions have the same sign (i.e., there is no frustration).

It should be emphasized here that the approach sketched in this section relies on a few additional approximations besides the LSDA to the DFT as routinely applied to zero-temperature properties of itinerant magnets. The major assumption is certainly the identification of the classical local moment directions $e_{R}$ with the only degrees of freedom relevant for the spin-dynamics and the finite-temperature statistical properties. A thorough discussion of this point goes beyond the scope of the present review and the reader is referred to the existing literature, covering both older phenomenological approaches $[45,46]$ as well as more recent justifications of the adiabatic spin-dynamics within the DFT $[26,34,35,36,37,38]$. Having in mind the broad variety of systems exhibiting a spontaneous magnetic order, a simple general criterion for validity of a complete neglect of other degrees of freedom, especially the variations of the local-moment magnitudes due to the Stoner particle-hole excitations (longitudinal spin fluctuations), can hardly be given. However, the assumption of adiabatic separation of the fast motion of individual electrons and the slow motion of spin-waves indicates that a necessary prerequisite is the existence of well-developed (atomic-like) moments the magnitude of which is little sensitive to effects of local magnetic environments and of el- 
evated temperatures. From a practical point of view, this sensitivity for a particular system can be estimated, e.g., by performing LSDA-DFT calculations for various collinear configurations, for spin-spirals [28, 29, 34] or for the disordered-local-moment (DLM) state with moments pointing to random directions [26, 47]. Ample experience shows that systems containing Mn and Fe atoms in a high-moment state and rare-earth magnets are favourable cases whereas the opposite limit contains Ni-based systems, the weakly ferromagnetic compounds $\mathrm{ZrZn}_{2}$ and $\mathrm{Sc}_{3} \mathrm{In}$, etc. Second basic assumption concerns the pair-wise exchange interaction as comprised in the classical EHH (2). While the use of a classical Hamiltonian is a direct consequence of the neglect of Stoner excitations and of mapping the DFT total energy, the full expansion of the total energy around the FM ground state yields an infinite series [48]. The pair interaction represents just its first term; higher terms of the expansion (1) (corresponding to triplet, biquadratic, ... interactions) can be calculated as well $[48,49]$ but they are usually neglected which is strictly correct only for long-wavelength magnons. Consequently, the spin-wave stiffness constants of ferromagnets resulting from the pair-wise EHH (2) are exact within the LSDA [33]. The third approximation is related to the use of the magnetic force theorem $[10,11]$ in deriving the simple formula for the pair interaction (5). The underlying physical picture assumes that the direction of the local magnetic moment and of the effective magnetic field acting on it coincide; this is fully justified, e.g., for two magnetic impurities in a paramagnetic host metal in the limit of a large separation of the impurity atoms. It has been proved that ferromagnets satisfy this assumption only if the exchange splitting is not too small as compared to the band-width [30] (see also section 5); a simple check can be based on evaluating the pair interactions (5) from other reference states (antiferromagnetic, DLM) besides the FM ground state [50]. Similar checks can be performed concerning the role of the neglected Stoner excitations. The imaginary part of frequency-dependent pair exchange interactions (Kohn-Sham dynamical transverse susceptibility) is related to the decay of magnons into the Stoner electron-hole continuum $[13,51]$ while an explicit temperature dependence of the pair exchange interactions can be obtained from an obvious modification of (5) (and the corresponding self-consistent electronic structure) by means of the Fermi-Dirac distribution function. The Stoner excitations can properly be described only using the full dynamical transverse susceptibility; its reliable evaluation is, however, a numerically very difficult task [52].

\section{Numerical implementation}

\subsection{Selfconsistent electronic structure}

Efficient evaluations of the pair exchange interactions (5) require a first-principle technique which provides the one-electron Green function in the real space. The results reported here are based on selfconsistent LSDA calculations using the all-electron nonrelativistic (scalar-relativistic) tight-binding linear muffin-tin orbital (TB-LMTO) method 
and the atomic-sphere approximation (ASA) [53, 54, 55], with the exchange-correlation potential parametrized according to reference [56]. The lattice parameters of the investigated systems were taken from experiment. The energy integrals over the occupied part of the valence band were expressed as integrals over a complex energy variable along a closed path $C$ starting and ending at the Fermi energy (with the occupied part of the valence band lying inside $C$ ). The integrals were numerically evaluated using the Gaussian quadrature method [54, 55]. Other Green function techniques, especially the the Korringa-Kohn-Rostoker (KKR) method [57, 58], are equally suitable in the present context.

Evaluation of the one-electron Green function $G^{\sigma}\left(\mathbf{r}, \mathbf{r}^{\prime} ; z\right)$ within the ASA reduces to the auxiliary (or KKR-ASA) Green function matrix $g_{\mathbf{R} L, \mathbf{R}^{\prime} L^{\prime}}^{\sigma}(z)$ defined in terms of the potential functions $P_{\mathbf{R} \ell}^{\sigma}(z)$ and the structure constants $S_{\mathbf{R} L, \mathbf{R}^{\prime} L^{\prime}}$ of the LMTO method as $[54,59]$

$$
g_{\mathbf{R} L, \mathbf{R}^{\prime} L^{\prime}}^{\sigma}(z)=\left\{\left[P^{\sigma}(z)-S\right]^{-1}\right\}_{\mathbf{R} L, \mathbf{R}^{\prime} L^{\prime}} .
$$

In the last equation, $L$ and $L^{\prime}$ are the angular momentum indices, $L=(\ell, m)$, the symbol $P^{\sigma}(z)$ denotes a diagonal matrix of potential functions defined as $P_{\mathbf{R} L, \mathbf{R}^{\prime} L^{\prime}}^{\sigma}(z)=$ $P_{\mathbf{R} \ell}^{\sigma}(z) \delta_{\mathbf{R} L, \mathbf{R}^{\prime} L^{\prime}}$ and $z$ is a complex energy variable. The quantities $P_{\mathbf{R} \ell}^{\sigma}(z)$ and $g_{\mathbf{R} L, \mathbf{R}^{\prime} L^{\prime}}^{\sigma}(z)$ can be expressed in any particular LMTO representation (canonical, screened); all derived physical quantities are invariant with respect to this choice. However, the most screened (tight-binding) representation is the best suited for most calculations and it has been employed in the present implementation. The energy dependence of the potential functions $P_{\mathbf{R} \ell}^{\sigma}(z)$ is parametrized in terms of three standard potential parameters, i.e., with the second-order accuracy $[53,54]$.

\subsection{Parameters of the classical Heisenberg Hamiltonian}

Substitution of the Green function $G^{\sigma}\left(\mathbf{r}, \mathbf{r}^{\prime} ; z\right)$ in the ASA (section 3.1) into (5) yields an expression suitable for computations $[11,13,15]$, namely,

$$
\begin{aligned}
J_{\mathbf{R R}^{\prime}} & =\frac{1}{4 \pi} \operatorname{Im} \int_{E_{\mathrm{B}}}^{E_{\mathrm{F}}} \operatorname{tr}_{L}\left[\Delta_{\mathbf{R}}\left(E^{+}\right) g_{\mathbf{R}^{\prime}}^{\uparrow}\left(E^{+}\right) \Delta_{\mathbf{R}^{\prime}}\left(E^{+}\right) g_{\mathbf{R}^{\prime} \mathbf{R}}^{\downarrow}\left(E^{+}\right)\right] \mathrm{d} E, \\
\Delta_{\mathbf{R}}(z) & =P_{\mathbf{R}}^{\uparrow}(z)-P_{\mathbf{R}}^{\downarrow}(z),
\end{aligned}
$$

where $\operatorname{tr}_{L}$ denotes the trace over the angular momentum index $L$, and the energy integration is performed between the bottom of the valence band $E_{\mathrm{B}}$ and the Fermi energy $E_{\mathrm{F}}$. In practice, this integral is replaced by a complex energy integral along the contour $C$ described in section 3.1. The quantities $g_{\mathbf{R R}^{\prime}}^{\sigma}(z)(\sigma=\uparrow, \downarrow)$ denote site-off-diagonal blocks of the auxiliary Green-function matrices with elements $g_{\mathbf{R} L, \mathbf{R}^{\prime} L^{\prime}}^{\sigma}(z)$ while $\Delta_{\mathbf{R}}(z)$ are diagonal matrices related to the potential functions $P_{\mathbf{R} \ell}^{\sigma}(z)$. The diagonal elements of $\Delta_{\mathbf{R}}(z)$ play a role of energy- and $\ell$-dependent exchange splittings on individual atoms while the expression (11) for the exchange interactions $J_{\mathbf{R R}^{\prime}}$ has a form of a bare static transversal susceptibility.

Well converged calculations of the exchange interactions $J_{\mathbf{R R}^{\prime}}$ for bulk metals with perfect translational symmetry for distances $d=\left|\mathbf{R}-\mathbf{R}^{\prime}\right|$ up to ten lattice parameters $a$ require high accuracy of both contour-integrations and the BZ-averages (inverse lattice Fourier transforms) defining the site-off-diagonal blocks $g_{\mathbf{R R}^{\prime}}^{\sigma}(z)[54,55]$. In particular, 
we have used typically a few millions of $\mathbf{k}$-points in the full BZ for the energy point on the contour $C$ closest to the Fermi energy, and the number of $\mathbf{k}$-points then progressively decreased for more distant energy points $[15,18]$ while the number of energy points on the countour was 20 to 40. A typical evaluation of exchange interactions requires a few hours on P4-based personal computers.

The calculated Heisenberg exchange parameters for bcc Fe (with experimental value of its lattice parameter $a$ ) are shown in figure 1. One can see dominating FM interactions for the first and second nearest-neighbour shells followed by weaker interactions of both signs and decreasing magnitudes for bigger distances $d=\left|\mathbf{R}-\mathbf{R}^{\prime}\right|$ (figure 1, left panel). The same qualitative features were found for other $3 d$ ferromagnets: fcc Co, fcc Ni [15] and hcp Co [60]. Note that the calculated exchange interactions depend on the type of the exchange-correlation potential [50] and on the inclusion of non-spherical parts of oneelectron potentials and densities [61]; these effects can be especially pronounced in some systems, e.g., in fcc Fe with atomic volume close to a crossover from a FM high-spin state to a FM low-spin state [61].
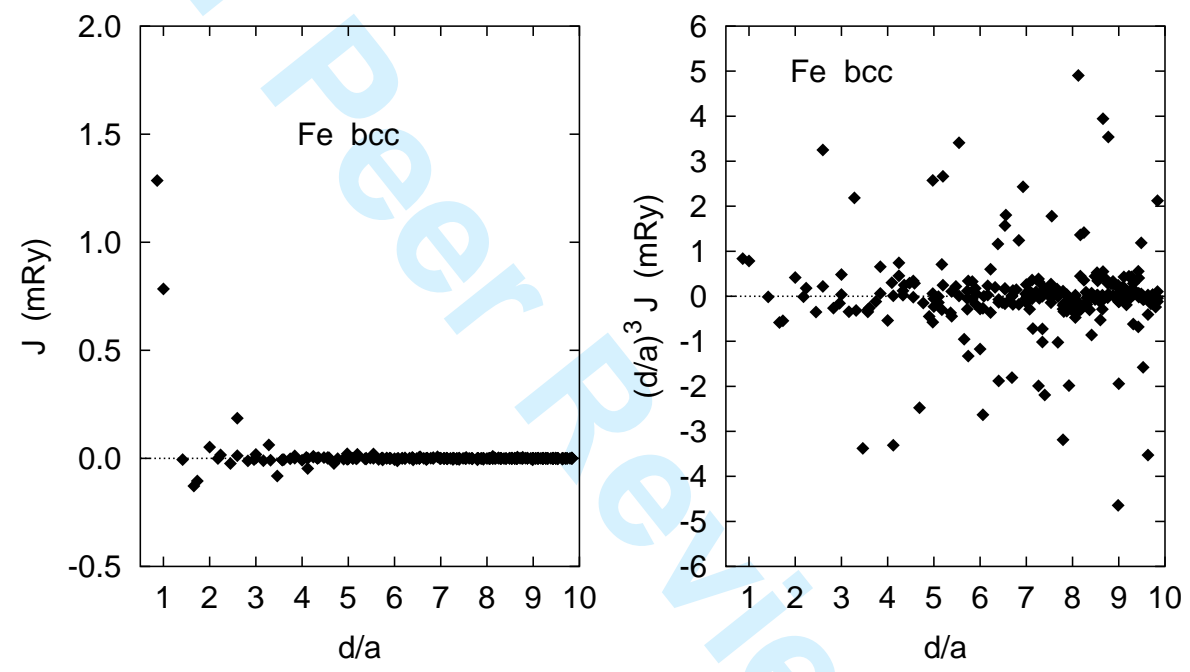

Figure 1: Exchange interactions $J_{\mathbf{R R}^{\prime}}$ for bcc Fe as a function of the distance $\left|\mathbf{R}-\mathbf{R}^{\prime}\right|=d$ without (left panel) and with (right panel) a prefactor $d^{3}$.

An analysis of the exchange interactions $J_{\mathbf{R R}^{\prime}}(11)$ in the limit of large distances $d=\left|\mathbf{R}-\mathbf{R}^{\prime}\right|$ has been given in reference [15] for a a single-band model using the stationaryphase approximation [62]. For a weak ferromagnet, one reveals a characteristic RudermanKittel-Kasuya-Yoshida (RKKY) asymptotic behaviour

$$
J_{\mathbf{R R}^{\prime}} \propto \frac{\sin \left[\left(\mathbf{k}_{\mathrm{F}}^{\uparrow}+\mathbf{k}_{\mathrm{F}}^{\downarrow}\right) \cdot\left(\mathbf{R}-\mathbf{R}^{\prime}\right)+\Phi\right]}{\left|\mathbf{R}-\mathbf{R}^{\prime}\right|^{3}},
$$

where $\mathbf{k}_{\mathrm{F}}^{\sigma}$ is a Fermi wave vector in a direction such that the associated group velocity is parallel to $\mathbf{R}-\mathbf{R}^{\prime}$, and $\Phi$ denotes a phase factor. The exchange interaction according to (12) has an oscillatory character with an envelope decaying as $\left|\mathbf{R}-\mathbf{R}^{\prime}\right|^{-3}$. On the other hand, for a strong ferromagnet with a fully occupied majority band the corresponding Fermi wave vector is imaginary, namely, $\mathbf{k}_{\mathrm{F}}^{\uparrow}=\mathrm{i} \mathbf{K}_{\mathrm{F}}^{\uparrow}$, and one obtains an exponentially 
damped RKKY behaviour [15, 63]

$$
J_{\mathbf{R R}^{\prime}} \propto \frac{\sin \left[\mathbf{k}_{\mathrm{F}}^{\downarrow} \cdot\left(\mathbf{R}-\mathbf{R}^{\prime}\right)+\Phi\right] \exp \left[-\mathbf{K}_{\mathrm{F}}^{\uparrow} \cdot\left(\mathbf{R}-\mathbf{R}^{\prime}\right)\right]}{\left|\mathbf{R}-\mathbf{R}^{\prime}\right|^{3}} .
$$

The qualitative features of these RKKY-type oscillations will not be changed in realistic ferromagnets. This is illustrated for bcc Fe (weak ferromagnet) in figure 1 (right panel) which proves undamped oscillations of the quantity $\left|\mathbf{R}-\mathbf{R}^{\prime}\right|^{3} J_{\mathbf{R R}^{\prime}}$. The complex oscillatory behaviour is due to the existence of both the majority and minority Fermi surfaces and the presence of various directions in the bcc-lattice which sample different $\mathbf{k}_{\mathrm{F}}^{\sigma}$-vectors. It should be noted that due to the $s p$ - $d$ hybridization no itinerant ferromagnet is a truly strong ferromagnet - the only exceptions are half-metallic ferromagnets.

\subsection{Magnetic properties from the Heisenberg Hamiltonian}

The RKKY-like asymptotic behaviour (12) leads to numerical difficulties in calculations of the magnon spectra and the spin-wave stiffness constants. The lattice Fourier transform of the exchange interactions (6) is not an absolutely convergent sum and its convergence with respect to the number of shells included has to be carefully checked. Note, however, that the lattice sum of $\left|J_{\mathbf{0 R}}\right|^{2}$ does converge so that $J(\mathbf{q})$ is defined unambiguously in the $L^{2}$ sense.

The lattice sum for the spin-wave stiffness constant (7) is not convergent at all, and the values of $D$ as functions of a cut-off distance $d_{\max }$ exhibit undamped oscillations for all three cubic $3 d$ ferromagnets [15]. This is a direct consequence of the RKKY asymptotics of the exchange interactions (12) which in turn reflects Fermi surface properties of metallic ferromagnets. To resolve this difficulty we suggested to regularize the original expression (7) by replacing it by the formally equivalent expression which is, however, numerically convergent

$$
\begin{aligned}
D(\eta) & =\frac{2}{3 M} \lim _{d_{\max } \rightarrow \infty} \sum_{|\mathbf{R}|<d_{\max }}|\mathbf{R}|^{2} J_{\mathbf{0 R}} \exp (-\eta|\mathbf{R}| / a) \\
D & =\lim _{\eta \rightarrow 0} D(\eta)
\end{aligned}
$$

where $a$ is the lattice parameter. The quantity $\eta$ plays a role of a damping parameter which makes the lattice sum absolutely convergent. It can be shown that the quantity $D(\eta)$ is a smooth function of the variable $\eta$ for any value $\eta>0$ and it can be extrapolated to the value $\eta=0$. We therefore perform calculations for a set of values $\eta \in\left(\eta_{\min }, \eta_{\max }\right)$ for which $D(\eta)$ is a smooth function with a well defined limit for large $d_{\max }$. The limit $\eta \rightarrow 0$ is then determined at the end of calculations by a quadratic least-square extrapolation method [15]. Note that these convergence problems are less serious in half-metallic magnets due to the exponential damping described by (13).

Direct calculations of the Curie temperatures in the MFA according to (8) face convergence problems similar to the magnon spectra. Alternatively, one can evaluate the on-site exchange parameter $J^{0}$ using a sum rule valid also for systems without translational periodicity [11]:

$$
J_{\mathbf{R}}^{0}=\sum_{\mathbf{R}^{\prime}} J_{\mathbf{R R}^{\prime}}
$$




$$
\begin{aligned}
& \text { Philospphical Magazine \& Philosophical Magazine Letters } \\
& \begin{aligned}
=-\frac{1}{4 \pi} \operatorname{Im} & \int_{E_{\mathrm{B}}}^{\mu_{\mathrm{F}}} \operatorname{tr}_{L}\left\{\Delta_{\mathbf{R}}\left(E^{+}\right)\left[g_{\mathbf{R} \mathbf{R}}^{\uparrow}\left(E^{+}\right)-g_{\mathbf{R} \mathbf{R}}^{\downarrow}\left(E^{+}\right)\right]\right. \\
& \left.+\Delta_{\mathbf{R}}\left(E^{+}\right) g_{\mathbf{R} \mathbf{R}}^{\uparrow}\left(E^{+}\right) \Delta_{\mathbf{R}}\left(E^{+}\right) g_{\mathbf{R} \mathbf{R}}^{\downarrow}\left(E^{+}\right)\right\} \mathrm{d} E .
\end{aligned}
\end{aligned}
$$

This sum rule involves only the site-diagonal blocks of the auxiliary Green functions and its reliable evaluation for perfect crystals requires only a few thousands of $\mathbf{k}$-points in the irreducible part of the BZ, i.e., accuracy usual in most selfconsistent LSDA calculations.

Another numerical problem is encountered in computations of the Curie temperature in the RPA due to the singularity of the averaged function in (9) for $|\mathbf{q}| \rightarrow 0$. We have therefore calculated $T_{\mathrm{C}}^{\mathrm{RPA}}$ using the expression

$$
\begin{aligned}
\left(k_{\mathrm{B}} T_{\mathrm{C}}^{\mathrm{RPA}}\right)^{-1} & =-\frac{3}{2} \lim _{z \rightarrow 0} G^{\mathrm{m}}(z), \\
G^{\mathrm{m}}(z) & =\frac{1}{N} \sum_{\mathbf{q}}\left[z-J^{0}+J(\mathbf{q})\right]^{-1},
\end{aligned}
$$

where $z$ is a complex energy variable and the quantity $G^{\mathrm{m}}(z)$ is a magnon Green function corresponding (up to the prefactor $4 / M$ ) to the magnon dispersion law (6). The magnon Green function was evaluated for energies $z$ in the complex energy plane and its value for $z=0$ was obtained using an analytical continuation technique [64].

The real-space method presented here allows to get a set of pair interactions even for very distant atoms only from a single selfconsistent calculation. It has therefore been used independently in a number of papers for very different systems $[65,66,67$, 68]. A widely used alternative approach, the so-called frozen-magnon approach, relies on constrained DFT calculations for true spin spirals and subsequent derivation of the coupling constants from the total energies of the spirals [34, 41, 69, 70]. The frozenmagnon approach can be implemented with the magnetic force theorem [71] or it can be formulated in terms of torques instead of total energies [72]. The real-space and frozenmagnon approaches are formally equivalent to each other. The quantities that are directly calculated (the pair exchange interactions $J_{\mathbf{R R}^{\prime}}$ in the former case, the magnon energies $E(\mathbf{q})$ 's in the latter) are related to each other by a lattice Fourier transformation (6). For this reason, their advantages and disadvantages refer mainly to their computational efficiency. For calculations of spin-wave dispersion curves (for a moderate number of $\mathbf{q}$ points) and of the spin-wave stiffness $D$, the frozen-magnon approach is superior, since it does not require to perform the Fourier transformation and the delicate analysis explained in section 3.3. The real-space approach seems to be more efficient for calculations of the Curie temperature, where the MFA estimation can be obtained from (8) and the sum rule (15), i.e., from a single real-space calculation, whereas a BZ-average of the magnon energies $E(\mathbf{q})$ is required in the frozen-magnon approach. The real-space technique is better also when a fine scan over the full BZ is needed like, e.g., in RPA calculations of the Curie temperature (9), or in searching for instabilities of the FM state (see section 4.2), since the set of calculated $J_{\mathbf{R R}^{\prime}}$ for typically 200 shells provides a fast and accurate parametrization of $J(\mathbf{q})$, which reduces considerably the computational effort. In general, the real-space approach has obvious advantages for systems with broken translational symmetry (random alloys, surfaces, thin films, multilayers) while the reciprocal-space approach is more natural for systems with full three-dimensional translational symmetry. 


\section{Page 27 of ${ }^{58}$ Applications}

\subsection{Transition metals}

Calculated magnon energy spectrum $E(\mathbf{q})$ for bcc Fe (by employing the pair exchange interactions up to 246 shells) is presented in figure 2. Corresponding plots of $E(\mathbf{q})$ for fcc Co and $\mathrm{Ni}$ [15] exhibit parabolic, almost isotropic behaviour for long wavelengths. On the contrary, in bcc Fe we observe some anisotropy of $E(\mathbf{q})$, i.e., $E(\mathbf{q})$ increases faster along the $\Gamma-N$ direction and more slowly along the $\Gamma-P$ direction. In agreement with references $[34,69,70]$ we observe local minima around the point $H$ along $\Gamma-H$ and $H-N$ directions in the range of short wavelengths. They are indications of the so-called Kohn anomalies which are due to long-range RKKY interactions similarly like Kohn-Migdal anomalies in phonon spectra are due to long-range interactions mediated by Friedel oscillations. It should be mentioned that the minima in dispersion curve of bcc Fe appear only if the summation in (6) is done over a sufficiently large number of shells, in the present case for more than 45 shells.

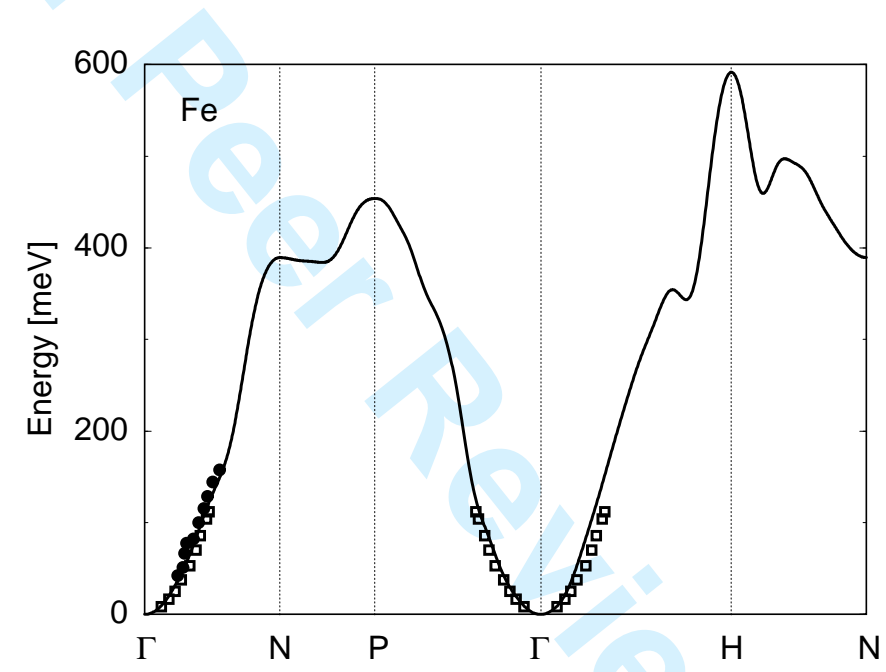

Figure 2: Magnon dispersion law along high-symmetry lines in the Brillouin zone of bcc Fe (fill line) compared to experiment (filled circles: pure Fe at $10 \mathrm{~K}$ [73], empty squares: $\mathrm{Fe}(12 \% \mathrm{Si})$ at room temperature [74]).

Present results for dispersion relations compare well with available experimental data of measured spin-wave spectra for Fe and $\mathrm{Ni}[73,74,75]$. For low-lying part of spectra there is also a good agreement of present results with those of references [34, 70] obtained using the frozen-magnon approach. There are, however, differences for a higher part of spectra, in particular for the magnon bandwidth of bcc Fe which can be identified with the value of $E(\mathbf{q})$ evaluated at the high-symmetry point $\mathbf{q}=\mathrm{H}$ in the bcc BZ. The origin of this disagreement is unclear; it may partly be ascribed to the third-order accuracy of the parametrization of the potential functions $P_{\mathbf{R} \ell}^{\sigma}(z)$ in reference [70], or to the true spin spirals in reference [34] that are more appropriate for high-energy excitations. We have carefully checked the convergence of the magnon dispersion laws $E(\mathbf{q})$ with the number of shells included in (6) and it was found to be weak for $50-70$ shells and more, i.e., for the cut-off distance $d_{\max } \geq 6 a$. 
The results for spin-stiffness coefficient $D$ calculated for the three cubic FM metals are summarized in table 1 together with available experimental data $[76,77,78]$. There is a reasonable agreement between theory and experiment for bcc Fe and fcc Co but the theoretical values of $D$ are considerably overestimated for fcc $\mathrm{Ni}$. It should be noted that measurements refer to the hcp Co while the present calculations were performed for fcc Co. A similar agreement between calculated and measured spin-wave stiffness constants was obtained by Halilov et al [34] using the frozen-magnon approach. Our results are also in accordance with those obtained by van Schilfgaarde and Antropov [70] who used the spin-spiral calculations to overcome the problem of evaluation of $D$ from (7). On the other hand, this problem was overlooked in references [11, 65, 51] so that a good agreement of $D$, calculated for a small number of coordination shells, with experimental data seems to be fortuitous. Finally, the results of Brown et al [79] obtained by the layer KKR method in the frozen-potential approximation are underestimated for all metals and the best agreement is obtained for $\mathrm{Ni}$.

Table 1: Calculated spin-wave stiffness constants $\left(D_{\mathrm{th}}\right)$ and Curie temperatures $\left(T_{\mathrm{C}}^{\mathrm{MFA}}\right.$ and $T_{\mathrm{C}}^{\mathrm{RPA}}$ ) for FM $3 d$ transition metals and their comparison with experimental values ( $D_{\exp }$ and $\left.T_{\mathrm{C} \text { exp }}\right)$. Calculations were performed with experimental values of lattice parameters.

\begin{tabular}{lcccrr}
\hline Metal & $D_{\text {th }}\left[\mathrm{meV} \cdot \AA^{2}\right]$ & $D_{\exp }\left[\mathrm{meV} \cdot \AA^{2}\right]$ & $T_{\mathrm{C}}^{\mathrm{MFA}}[\mathrm{K}]$ & $T_{\mathrm{C}}^{\mathrm{RPA}}[\mathrm{K}]$ & \multicolumn{1}{c}{$T_{\mathrm{C}, \exp }[\mathrm{K}]$} \\
\hline Fe bcc & $250 \pm 7$ & 280,330 & 1414 & $950 \pm 2$ & $1044-1045$ \\
Co fcc & $663 \pm 6$ & 580,510 & 1645 & $1311 \pm 4$ & $1388-1398$ \\
Ni fcc & $756 \pm 29$ & 555,422 & 397 & $350 \pm 2$ & $624-631$ \\
\hline
\end{tabular}

Calculated values of Curie temperatures for both MFA and RPA as well as corresponding experimental data are summarized in table 1. The MFA-values of Curie temperatures are overestimated for $\mathrm{Fe}$ and $\mathrm{Co}$, but underestimated for $\mathrm{Ni}$ in agreement with other calculations $[34,70]$. On the other hand, the results obtained using the RPA are in a good agreement with experiment for both fcc Co and bcc Fe, while the results for fcc Ni are even more underestimated. This is in agreement with the fact mentioned in section 2, namely that $T_{\mathrm{C}}^{\mathrm{RPA}}<T_{\mathrm{C}}^{\mathrm{MFA}}$. The present results for $\mathrm{Fe}$ and $\mathrm{Ni}$ agree reasonably with results of reference [80] using the spin-fluctuation theory and an improved statistical treatment in the framework of the Onsager cavity-field method.

In summary, we have found that calculated Curie temperatures and spin-wave stiffness constants agree well with experiment for Fe and Co, while less satisfactory results are obtained for $\mathrm{Ni}$, where the role of the Stoner excitations is much more important as compared to $\mathrm{Fe}$ and Co. In addition, the adiabatic approximation is less justified for $\mathrm{Ni}$, and, possibly, correlation effects beyond the LSDA play the more important role for this ferromagnet.

\subsection{Rare-earth metals}

Rare-earth (RE) metals represent a class of systems where the concept of atomic-like local moments is well justified due to highly localized $4 f$ orbitals. The standard LSDA, however, fails to describe correctly their ground-state properties [81]. We have treated 

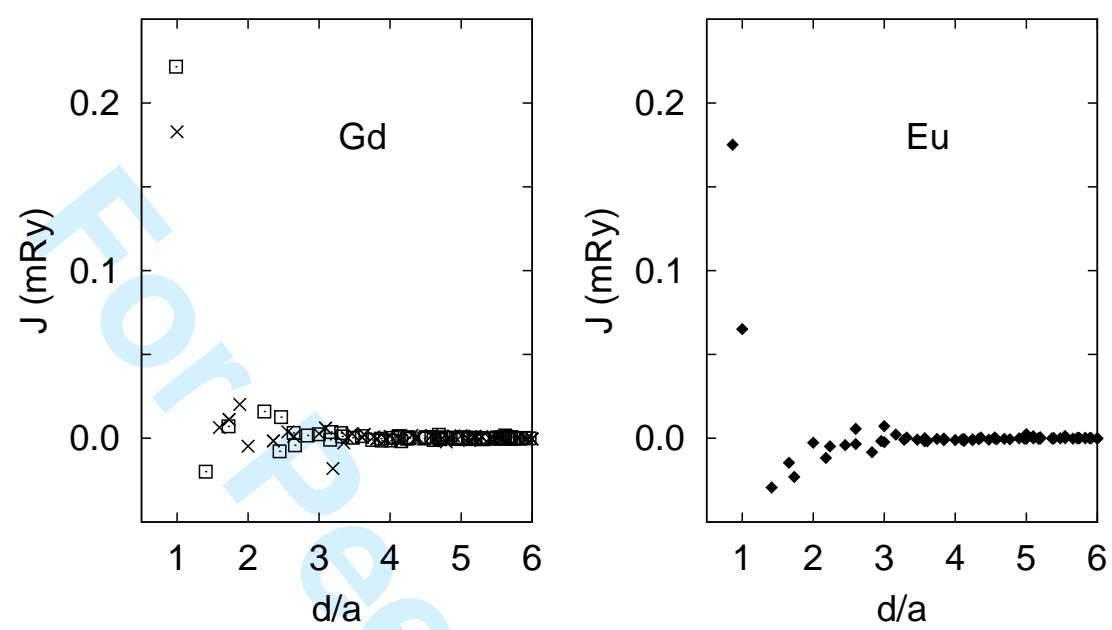

Figure 3: Exchange interactions $J_{\mathbf{R R}^{\prime}}$ for hcp Gd (left panel) and bcc Eu (right panel) as functions of the distance $\left|\mathbf{R}-\mathbf{R}^{\prime}\right|=d$. The crosses and squares in the left panel refer to pairs of sites $\mathbf{R}, \mathbf{R}^{\prime}$ lying in even (AA) and odd (AB) hcp(0001) planes, respectively.

The exchange interactions in Gd and Eu derived from selfconsistent calculations in a FM state are shown in figure 3 . Their distance-dependence is qualitatively similar to the $3 d$ transition metals, the magnitudes of the dominating RE nearest-neighbour interactions are, however, smaller by a factor of five, cf. figure 1. Moreover, there is a profound difference between the two $4 f$ metals concerning the contribution of the oscillating interactions between more distant atoms to the on-site exchange parameter $J^{0}$; well converged results of $J^{0}$ are obtained for cut-off distances $d_{\max }>5 a$ used in the real-space sum in (8) ( $a$ is the lattice parameter). In hcp Gd, the distant-pair interactions are not strong enough to destroy the FM spin structure, as indicated by a positive converged value of $J^{0}$ $\left(J^{0}=3.25 \mathrm{mRy}\right)[18]$. Note that the negative exchange interaction between the second Gd nearest neighbours is in qualitative agreement with experiment [87]. On the other hand, the contribution of more distant sites to $J^{0}$ is very important in the case of bcc Eu and it yields for the converged quantity a negligible resulting value $\left(J^{0}=-0.03 \mathrm{mRy}\right)$ [19]. Such a situation indicates an instability of the FM state with respect to a more complicated spin structure. This feature agrees qualitatively with an experimentally observed spin-spiral structure, the wave vector of which lies along the $\Gamma-\mathrm{H}$ direction in the bcc BZ $[88,89]$.

Calculations of the magnon spectra and the Curie temperature for hcp Gd require a trivial generalization of equations $(6,9)$ to the case of two equivalent atoms in the hcp unit cell $[42,90]$. The resulting Curie temperatures are given in table 2 together with the experimental value [91] while a comparison of the calculated magnon dispersion law 
Table 2: Calculated magnetic transition temperatures $\left(T^{\mathrm{MFA}}\right.$ and $\left.T^{\mathrm{RPA}}\right)$ and their comparison with experimental values $\left(T_{\exp }\right)$ for hcp Gd (Curie temperature) and bcc Eu (Néel temperature). Calculations were performed with experimental values of lattice parameters.

\begin{tabular}{lccc}
\hline Metal & $T^{\mathrm{MFA}}[\mathrm{K}]$ & $T^{\mathrm{RPA}}[\mathrm{K}]$ & $T_{\exp }[\mathrm{K}]$ \\
\hline Gd hcp & 334 & 301 & 293 \\
Eu bcc & 151 & 111 & 91 \\
\hline
\end{tabular}

with experiment [91] is presented in figure 4. The theoretical magnon spectra included finite temperature of the experiment $(T=78 \mathrm{~K})$ which leads within the RPA to a simple rescaling of the magnon energies proportionally to the temperature-dependent average magnetization [39]. The latter dependence was calculated in the RPA from the classical EHH [90].

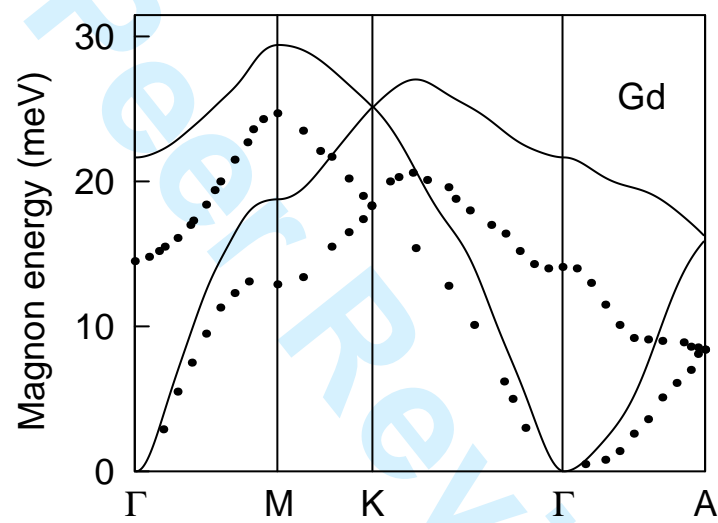

Figure 4: Magnon dispersion law along high-symmetry lines in the Brillouin zone of hcp Gd calculated for $T=78 \mathrm{~K}$ (full lines) and compared to experiment (filled circles reference [91]).

The calculated magnon energies are higher than experimental. A recent theoretical study by Halilov et al $[69,84]$ revealed that this effect can be partly explained by assumed intraatomic collinearity between the localized $4 f$-moment and the valence part of the local moment. Inclusion of a possible intraatomic non-collinearity between the localized and itinerant moments leads to a softening of the magnon energies, reducing them by a factor of 1.5 in the upper part of the spectrum. However, the lower part of the spectrum that is more important for an RPA estimation of the Curie temperature is less influenced by the non-collinearity. On the other hand, the calculated Curie temperatures both in the MFA and in the RPA agree very well with experiment (table 2). Having in mind a number of approximations used, a fortuitous cancellation of inaccuracies of different origins cannot be excluded; nevertheless, the degree of agreement of $T_{\mathrm{C}}$ 's in table 2 proves that the present approach based on interatomic exchange interactions represents a better starting point to RE magnetism than a theory based on intraatomic exchange integrals formulated in reference [92]. The latter scheme provided values of the Curie temperature for Gd in a 
wide interval $172-1002 \mathrm{~K}$, depending on further approximations employed.

Determination of the magnetic ground state of Eu from the EHH (2) is a difficult task in view of the highly-dimensional manifold of a priori possible states as well as a number of qualitatively different spin structures encountered in RE-based systems [91]. Here we consider only spin spirals specified by a single $\mathbf{q}$-vector as

$$
\overline{\mathbf{e}}_{\mathbf{R}}=(\sin (\mathbf{q} \cdot \mathbf{R}), 0, \cos (\mathbf{q} \cdot \mathbf{R}))
$$

since the spin structure observed for bcc Eu at low temperatures belongs to this class $[88,89]$. The minimum of the Hamiltonian $H_{\text {eff }}(2)$ corresponds then to the maximum of the lattice Fourier transform $J(\mathbf{q})(6)$. A scan over the whole BZ reveals that the absolute maximum of $J(\mathbf{q})$ is obtained for a vector $\mathbf{q}=\mathbf{Q}$ on the $\Gamma-\mathrm{H}$ line, namely, at $\mathbf{Q}=(1.63,0,0) a^{-1}$, see figure 5. The magnitude of $\mathbf{Q}$ determines the angle $\omega$ between magnetic moments in the neighbouring (100) atomic layers. In the present case, it is equal to $\omega=47^{\circ}$, in surprising agreement with experimental results which report the spin-spiral q-vector inside the $\Gamma-\mathrm{H}$ line and the angle per layer equal to $\omega_{\exp }=49^{\circ}$ [88] and $\omega_{\exp }=47.6 \pm 1.2^{\circ}[89]$.

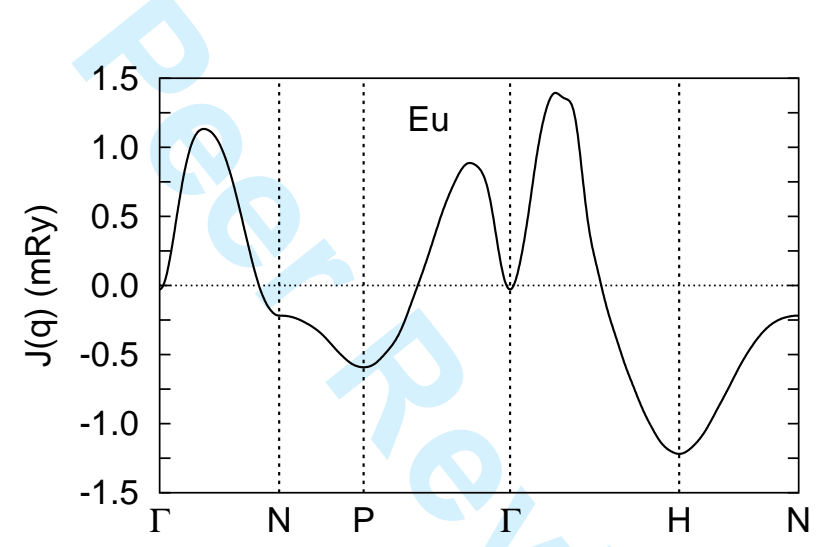

Figure 5: The lattice Fourier transform $J(\mathbf{q})$ of the exchange interactions in bcc Eu along high-symmetry lines in the Brillouin zone.

The resulting maximum $J(\mathbf{Q})$ can be used to get the Néel temperature in the MFA in complete analogy to (8) [91]:

$$
k_{\mathrm{B}} T_{\mathrm{N}}^{\mathrm{MFA}}=\frac{2}{3} J(\mathbf{Q})
$$

whereas the RPA leads to the following modification of $(9)[19,39]$ :

$$
\begin{aligned}
\left(k_{\mathrm{B}} T_{\mathrm{N}}^{\mathrm{RPA}}\right)^{-1} & =\frac{3}{4} \frac{1}{N} \sum_{\mathbf{q}}\left\{[J(\mathbf{Q})-J(\mathbf{q})]^{-1}+[W(\mathbf{q}, \mathbf{Q})]^{-1}\right\} \\
W(\mathbf{q}, \mathbf{Q}) & =J(\mathbf{Q})-\frac{1}{2} J(\mathbf{q}+\mathbf{Q})-\frac{1}{2} J(\mathbf{q}-\mathbf{Q}) .
\end{aligned}
$$

Both theoretical values and the experimental Néel temperature are given in table 2 . The MFA-value is substantially higher than experiment while the RPA reduces the theoretical value of $T_{\mathrm{N}}$ significantly so that a good agreement with experiment is obtained. Note that the present results are based on exchange interactions derived from a FM state 
which is not the true ground state; an extraction of the pair exchange interactions from the spin-spiral state (17) has not been done yet. The magnetic ground state and exchange interactions in bcc Eu have recently been studied using selfconsistent full-potential calculations and the LSDA+U scheme for spin spirals [93]. The obtained values of $J(\mathbf{q})$ compare reasonably well with the present results for q-vectors not far from the $\Gamma$-point while bigger differences are encountered at the BZ-boundary; these findings are consistent with expected validity of the present approach in the long-wavelength limit. The Néel temperatures derived in reference [93], $T_{\mathrm{N}}^{\mathrm{MFA}}=170 \mathrm{~K}$ and $T_{\mathrm{N}}^{\mathrm{RPA}}=112 \mathrm{~K}$, agree very well with the values based on EHH (table 2).

\subsection{Substitutional impurities and disordered alloys}

The present real-space approach to exchange interactions can be generalized to substitutionally disordered alloys either by using a supercell technique or by combining it with the coherent-potential approximation (CPA). Both alternatives have their own merits and drawbacks. The CPA takes properly into account the effects of finite lifetime of electronic states due to disorder but it has difficulties to include effects of varying local environments as well as of short-range order (both chemical and magnetic) on electronic properties.

In the following, we sketch the modification of the expression for the exchange interactions (11) to a random alloy within the LMTO-CPA formalism [54, 55, 94]. We assume that the lattice sites $\mathbf{R}$ are randomly occupied by alloy components $Q=A, B, \ldots$, with concentrations $c_{\mathbf{R}}^{Q}$. We neglect any correlations between occupations of different lattice sites and we neglect local environment effects, i.e., the LSDA selfconsistent potentials inside $\mathbf{R}$-th cell depend solely on occupation of the site $\mathbf{R}$ by an atom $Q=A, B, \ldots$.

The CPA-configurational average of the auxiliary Green function (10) can be written as

$$
\left\langle g_{\mathbf{R R}^{\prime}}(z)\right\rangle=\bar{g}_{\mathbf{R R}^{\prime}}(z)=\left\{[\mathcal{P}(z)-S]^{-1}\right\}_{\mathbf{R R}^{\prime}},
$$

where the spin index $\sigma$ is omitted, $S$ is the structure constant matrix and $\mathcal{P}(z)$ is a non-random site-diagonal matrix of coherent potential functions $\mathcal{P}_{\mathbf{R}}(z)$ attached to individual lattice sites which describe effective atoms forming an effective CPA medium. The coherent potential functions satisfy a set of selfconsistency conditions (Soven equation) which guarantees that average single-site scattering due to real atoms with respect to the effective medium vanishes.

The CPA leads also to conditional averages of individual blocks of the Green functions. The site-diagonal block $g_{\mathbf{R} \mathbf{R}}(z)$ of the Green function averaged over all alloy configurations with site $\mathbf{R}$ occupied by an atom $Q$ is given by

$$
\bar{g}_{\mathbf{R} \mathbf{R}}^{Q}(z)=\bar{g}_{\mathbf{R R}}(z) f_{\mathbf{R}}^{Q}(z)=\tilde{f}_{\mathbf{R}}^{Q}(z) \bar{g}_{\mathbf{R R}}(z),
$$

where the prefactors $f_{\mathbf{R}}^{Q}(z)$ and $\tilde{f}_{\mathbf{R}}^{Q}(z)$ are defined as

$$
\begin{aligned}
& f_{\mathbf{R}}^{Q}(z)=\left\{1+\left[P_{\mathbf{R}}^{Q}(z)-\mathcal{P}_{\mathbf{R}}(z)\right] \bar{g}_{\mathbf{R} \mathbf{R}}(z)\right\}^{-1} \\
& \tilde{f}_{\mathbf{R}}^{Q}(z)=\left\{1+\bar{g}_{\mathbf{R} \mathbf{R}}(z)\left[P_{\mathbf{R}}^{Q}(z)-\mathcal{P}_{\mathbf{R}}(z)\right]\right\}^{-1}
\end{aligned}
$$

Similarly, the site-off-diagonal block $g_{\mathbf{R}^{\prime}}(z)$ averaged over all alloy configurations with two sites $\mathbf{R} \neq \mathbf{R}^{\prime}$ occupied respectively by atomic species $Q$ and $Q^{\prime}$ is given by

$$
\bar{g}_{\mathbf{R} \mathbf{R}^{\prime}}^{Q Q^{\prime}}(z)=\tilde{f}_{\mathbf{R}}^{Q}(z) \bar{g}_{\mathbf{R} \mathbf{R}^{\prime}}(z) f_{\mathbf{R}^{\prime}}^{Q^{\prime}}(z)
$$


Derivation of the conditionally averaged pair exchange interaction between two sites $\mathbf{R} \neq \mathbf{R}^{\prime}$ occupied respectively by components $Q$ and $Q^{\prime}$ can be performed similarly like in the case without substitutional randomness by employing the magnetic force theorem [11] and the so-called vertex-cancellation theorem [95, 96]. It leads to an expression (see also [97])

$$
\begin{aligned}
\bar{J}_{\mathbf{R} \mathbf{R}^{\prime}}^{Q Q^{\prime}} & =\frac{1}{4 \pi} \operatorname{Im} \int_{E_{\mathrm{B}}}^{E_{\mathrm{F}}} \operatorname{tr}_{L}\left[\Delta_{\mathbf{R}}^{Q}\left(E^{+}\right) \bar{g}_{\mathbf{R} \mathbf{R}^{\prime}}^{Q Q^{\prime},}\left(E^{+}\right) \Delta_{\mathbf{R}^{\prime}}^{Q^{\prime}}\left(E^{+}\right) \bar{g}_{\mathbf{R}^{\prime} \mathbf{R}}^{Q^{\prime} Q, \downarrow}\left(E^{+}\right)\right] \mathrm{d} E, \\
\Delta_{\mathbf{R}}^{Q}(z) & =P_{\mathbf{R}}^{Q, \uparrow}(z)-P_{\mathbf{R}}^{Q, \downarrow}(z),
\end{aligned}
$$

which is fully analogous to (11). The conditional average of the on-site exchange interaction (15) yields a formula

$$
\begin{aligned}
\bar{J}_{\mathbf{R}}^{0, Q}=-\frac{1}{4 \pi} \operatorname{Im} & \int_{E_{\mathrm{B}}}^{E_{\mathrm{F}}} \operatorname{tr}_{L}\left\{\Delta_{\mathbf{R}}^{Q}\left(E^{+}\right)\left[\bar{g}_{\mathbf{R} \mathbf{R}}^{Q, \uparrow}\left(E^{+}\right)-\bar{g}_{\mathbf{R} \mathbf{R}}^{Q, \downarrow}\left(E^{+}\right)\right]\right. \\
& \left.+\Delta_{\mathbf{R}}^{Q}\left(E^{+}\right) \bar{g}_{\mathbf{R} \mathbf{R}}^{Q, \uparrow}\left(E^{+}\right) \Delta_{\mathbf{R}}^{Q}\left(E^{+}\right) \bar{g}_{\mathbf{R} \mathbf{R}}^{Q, \downarrow}\left(E^{+}\right)\right\} \mathrm{d} E .
\end{aligned}
$$

It should be noted, however, that the sum rule for the averaged pair and on-site interactions,

$$
\bar{J}_{\mathbf{R}}^{0, Q}=\sum_{\mathbf{R}^{\prime} Q^{\prime}} \bar{J}_{\mathbf{R} \mathbf{R}^{\prime}}^{Q Q^{\prime}} c_{\mathbf{R}^{\prime}}^{Q^{\prime}}
$$

which can be easily obtained from the corresponding sum rule (15), valid for any configuration of the alloy, is not exactly satisfied by the expressions (24) and (25). According to our experience, the two sides of (26) deviate up to $15 \%$ for typical binary transition-metal alloys $(\mathrm{FeV}, \mathrm{FeAl})$. This violation of an important sum rule indicates that vertex corrections must be taken into account in averaging exchange interactions in random alloys. On the other hand, the small relative difference of both sides of the sum rule (26) proves that the role of vertex corrections for exchange interactions is less significant than in transport properties, as argued in reference [98]. It can be expected that the vertex corrections are less important for the pair exchange interactions (especially between more distant sites) than for the on-site exchange interactions, but a thorough analysis of this point remains yet to be performed. A recent study using a supercell approach for random fcc $\mathrm{Fe}_{0.5} \mathrm{Ni}_{0.5}$ alloys proves that the CPA-averaged exchange interactions (24) agree reasonably well with averages from a 16-atom supercell [99].

Let us now consider the case of two isolated impurities in a non-magnetic host. The exchange interaction between two impurity sites $\mathbf{R} \neq \mathbf{R}^{\prime}$ can be calculated exactly and compared to the low-concentration limit of the CPA expression (24). The latter case corresponds to a binary alloy with $c_{\mathbf{R}}^{A} \rightarrow 0$ and $c_{\mathbf{R}}^{B} \rightarrow 1$ for all lattice sites, with spinpolarized impurity potential functions $P_{\mathbf{R}}^{\text {imp }, \sigma}(z)=P_{\mathbf{R}}^{A, \sigma}(z)$ and the coherent potential functions $\mathcal{P}_{\mathbf{R}}^{\sigma}(z) \rightarrow P_{\mathbf{R}}^{0}(z)$ where $P_{\mathbf{R}}^{0}(z)=P_{\mathbf{R}}^{B}(z)$ are the non-spin-polarized host potential functions. The average Green function is substituted by that of the non-magnetic nonrandom host, $\bar{g}_{\mathbf{R R}^{\prime}}^{\sigma}(z) \rightarrow g_{\mathbf{R}^{\prime}}^{0}(z)$. The impurity-impurity exchange interactions are given by

$$
\begin{aligned}
J_{\mathbf{R}^{\prime}} & =\frac{1}{4 \pi} \operatorname{Im} \int_{E_{\mathrm{B}}}^{E_{\mathrm{F}}} \operatorname{tr}_{L}\left[\tilde{\Delta}_{\mathbf{R}}\left(E^{+}\right) g_{\mathbf{R}_{\mathbf{R}^{\prime}}}^{0}\left(E^{+}\right) \tilde{\Delta}_{\mathbf{R}^{\prime}}\left(E^{+}\right) g_{\mathbf{R}^{\prime} \mathbf{R}}^{0}\left(E^{+}\right)\right] \mathrm{d} E, \\
\tilde{\Delta}_{\mathbf{R}}(z) & =\tau_{\mathbf{R}}^{\uparrow}(z)-\tau_{\mathbf{R}}^{\downarrow}(z), \\
\tau_{\mathbf{R}}^{\sigma}(z) & =\left[P_{\mathbf{R}}^{\mathrm{imp}, \sigma}(z)-P_{\mathbf{R}}^{0}(z)\right]\left\{1+g_{\mathbf{R} \mathbf{R}}^{0}(z)\left[P_{\mathbf{R}}^{\mathrm{imp}, \sigma}(z)-P_{\mathbf{R}}^{0}(z)\right]\right\}^{-1},
\end{aligned}
$$


where the $\tau_{\mathbf{R}}^{\sigma}(z)$ denote single-site t-matrices of the impurity atoms. A conventional RKKY-expression is obtained by replacing the t-matrices $\tau_{\mathbf{R}}^{\sigma}(z)$ in $\tilde{\Delta}_{\mathbf{R}}(z)$ by the impurity potential functions $P_{\mathbf{R}}^{\mathrm{imp}, \sigma}(z)$. This is justified in the limit of a weak scattering, whereas the formula (27) is correct also for strong impurity scatterings and it modifies the phase and amplitude of the oscillations of the exchange interactions as compared to the conventional RKKY formula [100].
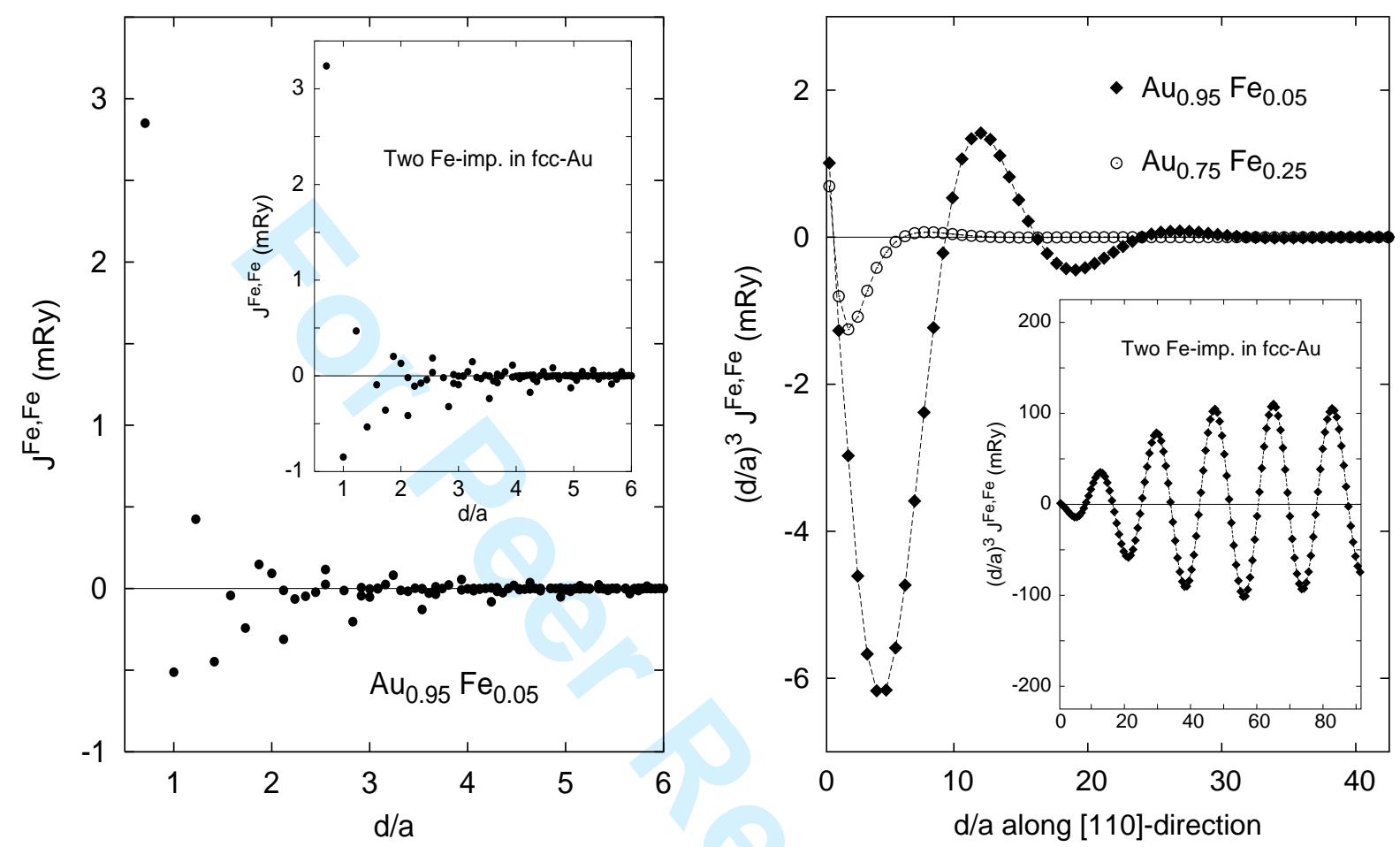

Figure 6: Exchange interactions between Fe atoms $\overline{\bar{J}_{\mathbf{R}}^{\prime}}$ as a function of the distance $\left|\mathbf{R}-\mathbf{R}^{\prime}\right|=d$ in Au-rich random fcc $\mathrm{Au}_{1-x} \mathrm{Fe}_{x}$ alloys: bare interactions for all neighbours (left panel) and interactions multiplied by $d^{3}$ for Fe atoms along the nearest-neighbour direction [110] (right panel). The insets show results for two Fe impurities in a pure fcc Au host.

The calculated exchange interactions for two Fe impurities in an fcc Au host are shown in figure 6 (inset in the left panel). The values of $J_{\mathbf{R R}^{\prime}}$ for impurities along the nearest-neighbour direction [110] (which gives the dominating contribution to exchange interactions in this case) were multiplied by the cube of the interatomic distance in order to illustrate the RKKY asymptotics for large distances (figure 6, inset in the right panel). However, it takes a few oscillations (a preasymptotic regime) before the asymptotic value of the oscillation amplitudes is reached. Very similar behaviour is obtained also for a textbook example of two $\mathrm{Mn}$ impurities in an fcc $\mathrm{Cu}$ host. The main difference is the character of the leading nearest-neighbour interactions: they are FM for Fe-impurities in the $\mathrm{Au}$ host, but antiferromagnetic for two neighbouring $\mathrm{Mn}$ atoms in $\mathrm{Cu}$.

The formalism can also be applied to magnetic impurities in spin-polarized hosts such as $3 d$ FM metals Fe, Co and Ni. Here the main interest concerns the exchange coupling of the impurity local moment to the bulk magnetization. The on-site exchange parameter for the impurity atoms, obtained from (25) in the low-concentration limit, represents a 

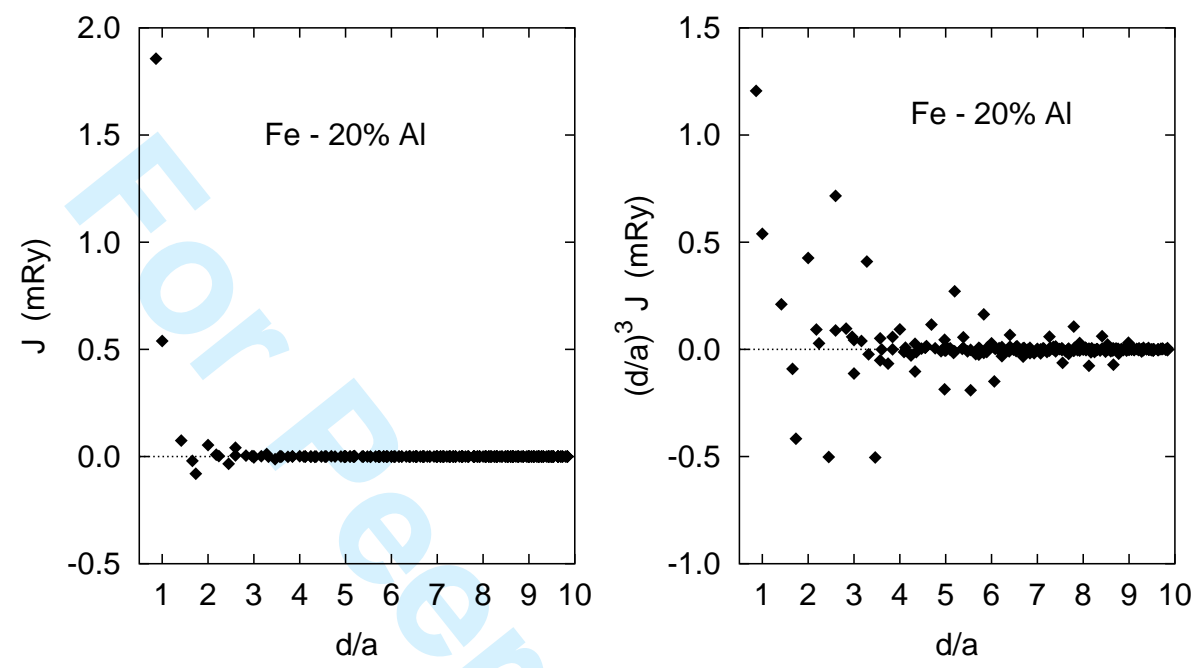

Figure 7: Exchange interactions $\bar{J}_{\mathbf{R R}^{\prime}}^{\mathrm{FeFe}}$ for a random bcc $\mathrm{Fe}_{0.8} \mathrm{Al}_{0.2}$ alloy as a function of the distance $\left|\mathbf{R}-\mathbf{R}^{\prime}\right|=d$ without (left panel) and with (right panel) a prefactor $d^{3}$.

An example of pair exchange interactions in a concentrated alloy is shown in figure 7 for Fe-Fe pairs in a disordered bcc $\mathrm{Fe}_{0.8} \mathrm{Al}_{0.2}$ system. The interactions are qualitatively similar to those in pure bcc Fe (figure 1), but a more careful analysis of the long-distance behaviour reveals exponentially damped RKKY-like oscillations (figure 7, right panel). This feature can be explained by damping of electron states due to the alloy disorder which leads to an exponential decay of site-off-diagonal blocks of the averaged Green functions $\bar{g}_{\mathbf{R}^{\prime}}^{\sigma}(z)$ with increasing distance $\left|\mathbf{R}-\mathbf{R}^{\prime}\right|$. It should be mentioned that this exponential damping refers only to averaged exchange interactions in contrast to those in each alloy configuration which exhibit a much slower decay for large interatomic separations (see reference [98] and references therein).

The exponential damping is also encountered in spin-glass systems such as AuFe or $\mathrm{CuMn}$; its presence is visible even for a very small but finite concentration of magnetic impurities. The bare exchange interactions for two $\mathrm{Fe}$ atoms in fcc-Au and in random $\mathrm{Au}_{0.95} \mathrm{Fe}_{0.05}$ alloy are shown in figure 6 (left panel). We observe the dominating FM nearest-neighbour interactions and a pronounced frustration of further exchange interactions. The magnetic frustration, defined as the competition between antiferromagnetic and FM interactions, and the substitutional (chemical) disorder are the essential ingredients of a spin glass. A detailed analysis [25] shows that the frustration in AuFe alloys vanishes gradually as the Fe-content increases and the system reaches a FM phase. Our results suggest that the exchange interactions in fcc-AuFe spin-glass alloys are much shorter-ranged than to be expected from ordinary RKKY interactions (see figure 6, right 
panel). As compared to the case of the very dilute limit, where the asymptotic RKKY behaviour was verified numerically (see the inset), already for $5 \%$ of Fe-impurities there is a pronounced exponential damping of calculated exchange interactions. Because the damping increases further with increasing Fe-content, the short-range theories [103] seem to be more appropriate for a study of the spin-glass properties in the AuFe metallic system. A more detailed discussion of the calculated exchange interactions in the AuFe spin glass can be found elsewhere [25]. Similar results, namely the damped RKKY interactions, were obtained in studies of the paramagnetic spin susceptibility and compositional correlation function in the DLM state [104] of the CuMn [105, 106] and AuFe [107] spin glasses. This agreement represents a rewarding feature since the latter approach [104] is not based on any particular model of magnetic interactions.

Calculation of the magnon spectra and related quantities from the EHH for disordered alloys represents a non-trivial task since the corresponding equation of motion for the twotime Green function for spin operators, obtained from the standard decoupling procedure for higher-order Green functions [39], contains a more complicated type of disorder than purely diagonal disorder. The magnons (and also phonons) in random alloys are featured by simultaneous presence of diagonal, off-diagonal and environmental disorder; the latter is closely related to the Goldstone theorem for these excitations. An extension of the CPA to this case has been studied since early 1970's. Two recent approaches are based on a cumulant expansion [108] and on an augmented-space formalism [109]; the former scheme is combined with the RPA and provides a value for the Curie temperature. Both formulations are rather complicated which allowed to perform numerical calculations for environmental disorder limited to nearest neighbours only, but they seem promising for future studies with true long-range interactions. The disorder can be taken into account exactly by Monte Carlo simulations using large simulation cells which can describe fluctuations beyond any effective-medium approach [24]; the combined effect of magnetic and chemical fluctuations can also be included within a real-space RPA [110].

The critical temperature of a random alloy in the MFA can be obtained in a way similar to that leading to (8). Let us restrict ourselves to the case of a homogeneous random alloy (with all lattice sites equivalent). In analogy to previous on-site exchange parameters $(8,26)$, one can introduce quantities

$$
\bar{J}^{0, Q Q^{\prime}}=\sum_{\mathbf{R}^{\prime} Q^{\prime}} \bar{J}_{\mathbf{R R}^{\prime}}^{Q Q^{\prime}}, \quad \mathcal{K}^{Q Q^{\prime}}=\bar{J}^{0, Q Q^{\prime}} c^{Q^{\prime}}
$$

where $\mathcal{K}^{Q Q^{\prime}}$ are effective exchange parameters among magnetic moments of the alloy constituents. The critical temperature is then equal to

$$
k_{\mathrm{B}} T_{\mathrm{cr}}^{\mathrm{MFA}}=\frac{2}{3} \kappa_{\max }
$$

where $\kappa_{\max }$ is the maximal eigenvalue of the matrix $\mathcal{K}^{Q Q^{\prime}}$. This type of estimation has been used for diluted magnetic semiconductors as described in section 4.4.

The expression for the alloy spin-wave stiffness constant is similar to (7), namely,

$$
D=\frac{2}{3 \bar{M}} \sum_{\mathbf{R}}|\mathbf{R}|^{2} \bar{J}_{\mathbf{0 R}}, \quad \bar{J}_{\mathbf{R R}^{\prime}}=\sum_{Q Q^{\prime}} c_{\mathbf{R}}^{Q} c_{\mathbf{R}^{\prime}}^{Q^{\prime}} \bar{J}_{\mathbf{R R}^{Q R^{\prime}}},
$$


where $\bar{M}$ and $\bar{J}_{\mathbf{R R}^{\prime}}$ denote the average alloy magnetic moment (per site) and the averaged exchange pair interactions, respectively [97]. The sum in (30) is absolutely convergent due to the exponential damping of the pair exchange interactions for $\left|\mathbf{R}-\mathbf{R}^{\prime}\right| \rightarrow \infty$.

\subsection{Diluted magnetic semiconductors}

Diluted magnetic semiconductors (DMS) represent a new class of materials with potential technological applications in spintronics. They have recently attracted much interest because of the hole-mediated ferromagnetism [111, 112, 113]. Curie temperatures higher than the room temperature are desirable for practical applications, whereas the currently prepared samples exhibit the $T_{\mathrm{C}}$ 's only slightly above $150 \mathrm{~K}$ [114]. The most frequently studied DMS is a III-V-based compound $\left(\mathrm{Ga}_{1-x} \mathrm{Mn}_{x}\right)$ As in the zinc-blende structure with Mn-concentration in the range $0<x<0.1$. Since $\mathrm{Mn}$ atoms are in a high-spin state in these systems, the above described formalism is well suited for reliable quantitative investigations of the exchange interactions and the Curie temperatures.
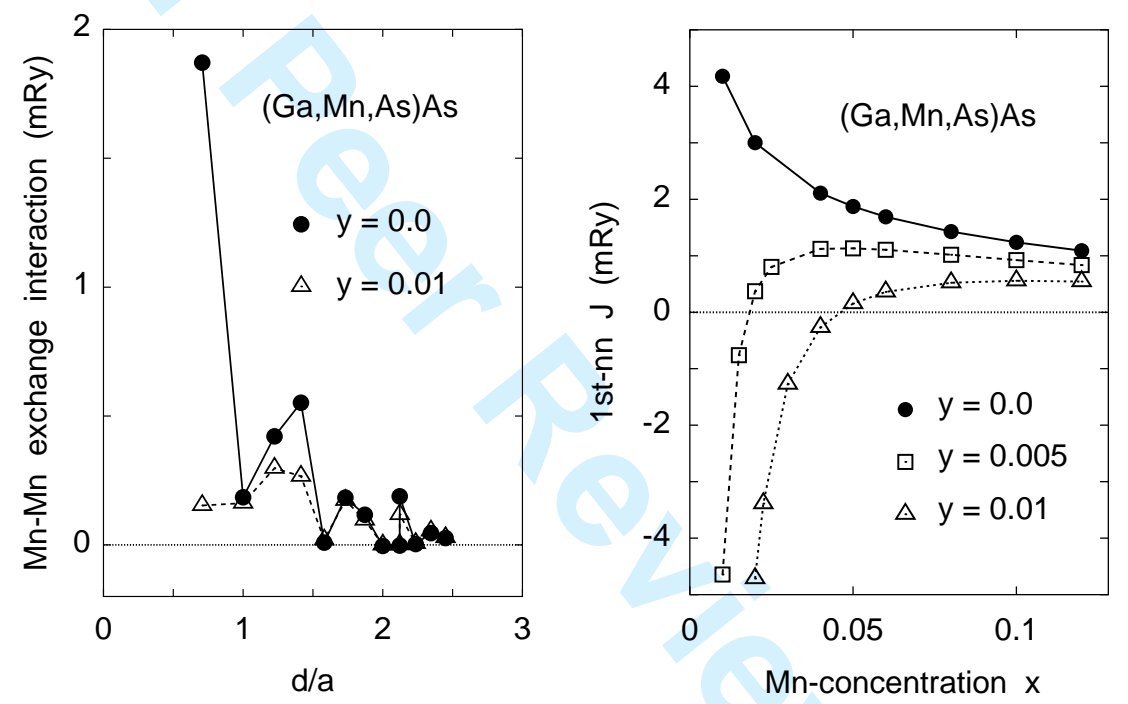

Figure 8: The Mn-Mn exchange interactions in $\left(\mathrm{Ga}_{0.95-y} \mathrm{Mn}_{0.05} \mathrm{As}_{y}\right)$ As as a function of the Mn-Mn distance $d$ (left panel) and the first nearest-neighbour Mn-Mn interaction in $\left(\mathrm{Ga}_{1-x-y} \mathrm{Mn}_{x} \mathrm{As}_{y}\right)$ As (right panel).

The (Ga,Mn)As compound is a substitutionally disordered system with Mn atoms substituting Ga atoms on the cation sublattice. Application of the TB-LMTO-CPA formalism to this system employs so-called empty spheres located at interstitial positions of GaAs semiconductor for matters of space filling, so that the zinc-blende structure is described in terms of four fcc sublattices with substitutional disorder only on the cation sublattice. The pair exchange interactions between $\mathrm{Mn}$ atoms $\bar{J}_{\mathbf{R R}^{\prime}}^{\mathrm{Mnn}}$ in the $\left(\mathrm{Ga}_{1-x} \mathrm{Mn}_{x}\right) \mathrm{As}$ alloy with $x=0.05$ are shown in figure 8 (left panel) [17, 22, 60]; interactions between the other components are much smaller and negligible concerning their possible influence on magnetic properties. The first nearest-neighbour interaction is positive and bigger than the (mostly positive) interactions between more distant Mn atoms. Analysis of the behaviour of $\bar{J}_{\mathbf{R R}^{\prime}}^{\mathrm{MnMn}}$ for large interatomic distances reveals exponentially damped RKKYlike oscillations [22] which have two origins: the effect of alloying which introduces an 
exponential damping in the site-off-diagonal blocks of the averaged Green functions (see section 4.3), and an additional exponential damping due to a half-metallic character of the system $[22,111]$, i.e., the alloy Fermi energy lies in a band gap of the minority band (see section 3.2).

The calculated Curie temperature in MFA for the $\left(\mathrm{Ga}_{0.95} \mathrm{Mn}_{0.05}\right)$ As alloy is around $300 \mathrm{~K}$, i.e., substantially higher than the experimental values $[112,114,115]$. There are several possible reasons for this discrepancy, namely, (i) the structural imperfections of the compound which reduce the number of holes in the valence band, (ii) the simplified treatment of the lattice disorder in the EHH (within the MFA), (iii) effects of electron correlations not included in the LSDA, (iv) variations of the pair exchange interactions due to random configurations (local environment effect) that are not captured by the present CPA-averaged interactions (24), and (v) nonnegligible atomic short-range order. The most probable candidates for structural imperfections are native defects, such as As-antisite atoms [111] and Mn-interstitial atoms [116]. The effect of lattice disorder on the Curie temperature can be included essentially exactly in the framework of supercell approaches $[24,110]$.

In the following, we demonstrate the effect of As-antisites on the exchange interactions of (Ga,Mn)As compounds [21, 22]. The combined effect of Mn-impurities and As-antisites can be simulated within the CPA using an alloy $\left(\mathrm{Ga}_{1-x-y} \mathrm{Mn}_{x} \mathrm{As}_{y}\right)$ As with $y$ denoting the As-antisite concentration. The influence of As-antisites on the Mn-Mn exchange interactions is shown in figure 8 (left panel): the positive values of $\bar{J}_{\mathbf{R}^{\prime}}^{\mathrm{MnMn}}$ are reduced due to the As-antisites; the most dramatic reduction is found for the dominating coupling between the nearest neighbours. The dependence of the nearest-neighbour Mn-Mn interaction on $x$ and $y$ is shown in figure 8 (right panel). For a fixed Mn-concentration $x$, the interaction decreases monotonously with increasing content of As-antisites $y$, ending finally at negative values. This change of sign correlates nicely with a predicted instability of the FM state with respect to formation of a state featured by disordered directions of the Mn-moments [17, 117]. A simple physical explanation of these effects is based on the component-resolved densities of states at the Fermi level $[117,118]$. In alloys without As-antisites, a very narrow impurity band due to $\mathrm{Mn}$ atoms with negative moments is formed just at $E_{\mathrm{F}}$, indicating that a flip of $\mathrm{Mn}$ local moment is energetically unfavorable. The presence of As-antisites leads to an upward shift of $E_{\mathrm{F}}$ and a complete filling of the impurity band, which reduces the large energy cost of Mn-moment reversal.

The Curie temperatures were estimated in the MFA $[17,22]$ as described in section 4.3. However, in view of the much bigger Mn-Mn interactions as compared to interactions between other constituent atoms, the Curie temperature comes out equal to

$$
k_{\mathrm{B}} T_{\mathrm{C}}^{\mathrm{MFA}}=\frac{2}{3} x \sum_{\mathbf{R}^{\prime}} \bar{J}_{\mathbf{R R}^{\prime}}^{\mathrm{MnMn}},
$$

where the lattice sites $\mathbf{R}, \mathbf{R}^{\prime}$ are confined to the cation fcc sublattice and $x$ denotes the Mn-concentration. The $T_{\mathrm{C}}^{\mathrm{MFA}}$ for a fixed $x$ is monotonously decreasing with increasing Asantisite concentration $y$ (see also figure 10, right panel), in analogy to the $y$-dependence of the first nearest-neighbour Mn-Mn interaction (figure 8, right panel). The $T_{\mathrm{C}}$ for a fixed $y$ exhibits a non-monotonous dependence on the Mn-content $x$ reaching a flat maximum for $x>0.1$. The latter behaviour results from an interplay of two effects: an increase of $T_{\mathrm{C}}^{\mathrm{MFA}}$ with increasing $x$, see (31), and the non-trivial dependence of the first nearestneighbour Mn-Mn interaction as a function of $(x, y)$, see figure 8 (right panel). Note, 


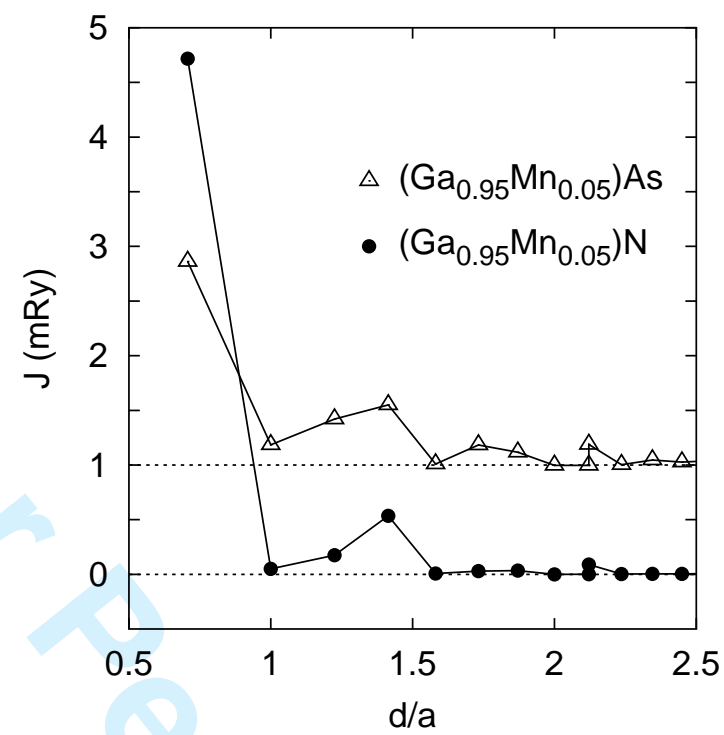

Figure 9: The Mn-Mn exchange interactions in $\left(\mathrm{Ga}_{0.95} \mathrm{Mn}_{0.05}\right) \mathrm{As}$ (open triangles) and $\left(\mathrm{Ga}_{0.95} \mathrm{Mn}_{0.05}\right) \mathrm{N}$ (full dots) as a function of the Mn-Mn distance $d$.

A combination of the frozen-magnon and supercell approaches was used to study Curie temperatures in (Ga,Mn)As (without structural defects) in the MFA and the RPA [71]. It yielded a non-monotonous dependence of the $T_{\mathrm{C}}$ on the Mn-concentration with the RPA values about $20 \%$ smaller than the MFA values; the latter compare well with the present results [22]. It should be noted, however, that the supercell approach was limited to a few special Mn-concentrations $(x=0.03125,0.0625,0.125,0.25)$ and that the first nearest-neighbour Mn-Mn interactions could not be determined due to the special atomic order of the supercells.

Probably the most reliable way of obtaining the Curie temperature from parameters of the EHH for a random system is the Monte Carlo (MC) simulation [120]; its recent applications indicate that previous MFA estimations of the $T_{\mathrm{C}}$ 's of DMS's should be taken with care $[24,121]$. The MC simulations take into account on equal footing both correlations of thermal fluctuations of the spin variables and the randomness of the alloy configurations. The latter is especially important for small concentrations of magnetic atoms where effects of magnetic percolation become important for the critical behaviour. This point is illustrated in figures 9 and 10 for the (Ga,Mn)As and (Ga,Mn)N alloys. The Mn-Mn exchange interactions in the former have broader spatial range than in the latter, see figure 9. This difference can be ascribed to the exponential damping of the exchange interactions due to the half-metallic nature of the DMS's and due to different band gaps: the band gap of the parent semiconductor GaN is wider than that of GaAs. The resulting Curie temperatures are plotted in figure 10. For the $\left(\mathrm{Ga}_{1-x-y} \mathrm{Mn}_{x} \mathrm{As}_{y}\right)$ As system, the MFA overestimates the MC-values by a factor of two, but it yields at least roughly correct concentration trends of the $T_{\mathrm{C}}$. The experimental data of Edmonds et al [114, 115] 

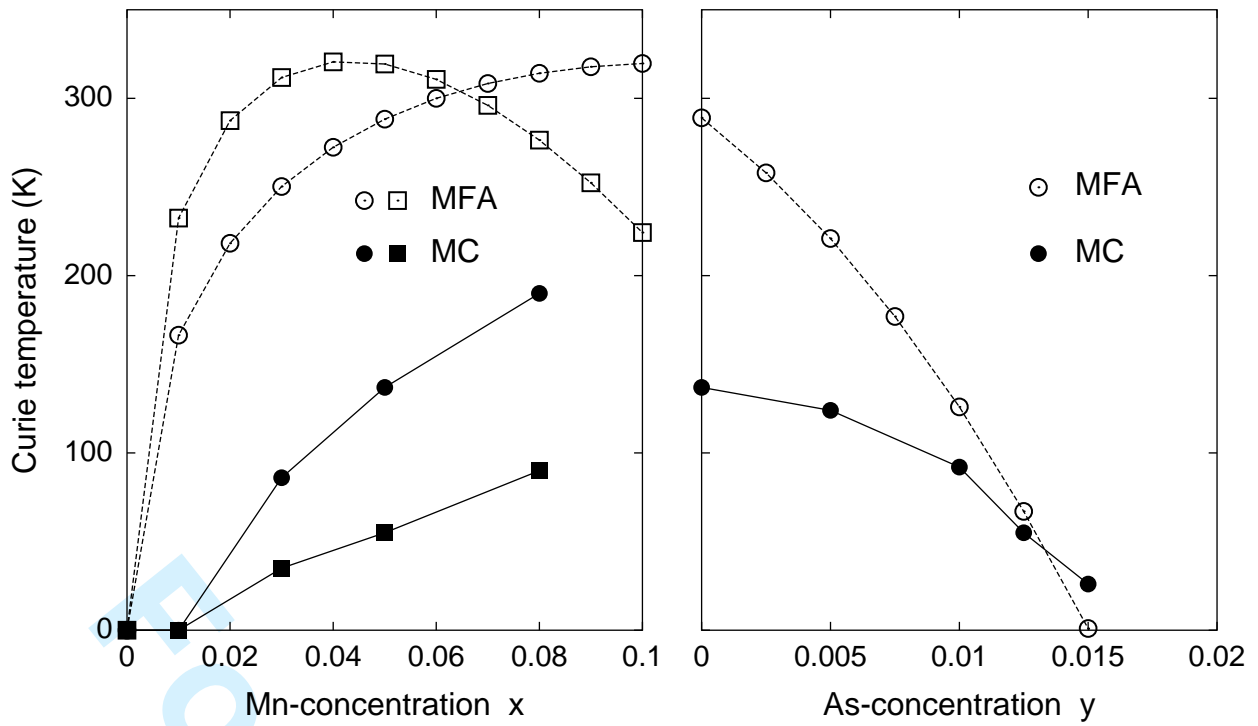

Figure 10: Left panel: Curie temperatures calculated for $\left(\mathrm{Ga}_{1-x} \mathrm{Mn}_{x}\right) \mathrm{As}$ (open circles - MFA, full circles - MC) and for $\left(\mathrm{Ga}_{1-x} \mathrm{Mn}_{x}\right) \mathrm{N}$ (open squares - MFA, full squares $\mathrm{MC}$ ) as functions of the Mn content $x$. Right panel: Curie temperatures calculated for $\left(\mathrm{Ga}_{0.95-y} \mathrm{Mn}_{0.05} \mathrm{As}_{y}\right)$ As (open circles - MFA, full circles - MC) as functions of the Asantisite content $y$.

for about $5 \%(8 \%)$ of $\mathrm{Mn}$ in GaAs host vary in the range $48-118 \mathrm{~K}(55-170 \mathrm{~K})$ depending on the sample preparation. The lower (upper) values correspond to as-grown (well annealed) samples with essential (negligible) presence of compensating defects, such as As-antisites and/or Mn-interstitials. The results of MC simulations in figure 10 (left panel) agree well with the measured data for annealed samples. On the other hand, the MFA fails completely for the $\left(\mathrm{Ga}_{1-x} \mathrm{Mn}_{x}\right) \mathrm{N}$ system by predicting a non-monotonous behaviour of the $T_{\mathrm{C}}^{\mathrm{MFA}}$ with a maximum above $300 \mathrm{~K}$ around $x \approx 0.04$, in contrast to the $\mathrm{MC}$ simulations that lead to a magnetic gap for small Mn-concentrations followed by a slow increase of $T_{\mathrm{C}}^{\mathrm{MC}}$ with increasing $x$. These results demonstrate that percolation becomes more important for systems with exchange interactions strongly localized in the real space; calculations based on the KKR-CPA method [121] as well as on the realspace RPA [110] confirm these conclusions. More details on the MC simulations including results for a $(\mathrm{Zn}, \mathrm{Cr}) \mathrm{Te}$ system have been published elsewhere [24].

Let us briefly discuss other possible sources of discrepancy between theoretical and experimental values of the Curie temperatures of the DMS. It has been shown by using the LSDA+U method that strong electron correlations due to the Mn-3d orbitals are important for the electronic structure and exchange interactions $[22,122,123,124]$. In particular, the value of $U=4 \mathrm{eV}$ (bringing the calculated local densities of states in a reasonable agreement with measured photoemission spectra) reduces the exchange interactions and the Curie temperature for $\mathrm{Ga}_{0.95} \mathrm{Mn}_{0.05} \mathrm{As}$ system, but leads to their increase in the case of $\mathrm{Ga}_{0.95} \mathrm{Mn}_{0.05} \mathrm{~N}$ alloy [22, 123]; a study of concentration trends for the two systems can be found in Ref. [125]. In both cases, the occupied Mn-3d peaks of the majority spin are shifted downwards as compared to the LSDA; 
the different behaviour of the two systems can be ascribed to differences in the electronic structure of Mn-atoms in the host semiconductors: Mn in GaAs is a shallow acceptor strongly interacting with the valence band, whereas Mn in $\mathrm{GaN}$ is a deep acceptor leading to an impurity level inside the band gap [124].

A recent study simulating the disorder in DMS in terms of large (128-250 atom) supercells and special quasirandom structures has addressed the role of local environment effects on exchange interactions and the Curie temperatures in (Ga,Mn)As, (Ga,Cr)As and ( $\mathrm{Ga}, \mathrm{Cr}) \mathrm{N}$ alloys [126]. The results reveal strong sensitivity of pair interactions to varying local environments; the $T_{\mathrm{C}}$ of $\mathrm{Ga}_{0.92} \mathrm{Mn}_{0.08} \mathrm{As}$ with environment-specific interactions is about $50 \mathrm{~K}$ smaller than that with configurationally-averaged interactions. The Curie temperature of this alloy has also been found strongly dependent on the degree of atomic short-range order: a tendency to clustering of Mn atoms on the cation sublattice leads to a decrease (of about $100 \mathrm{~K}$ ) of $T_{\mathrm{C}}$. The latter fact has been explained on the basis of percolation: increased clustering weakens links in magnetic percolation paths [126]. An opposite trend, namely an increase of $T_{\mathrm{C}}$ due to enhanced probability of Mn-Mn pairs on neighbouring cation sites, has been reported in a similar study of ( $\mathrm{Ga}, \mathrm{Mn}) \mathrm{As}$ and (Ga,Mn)N alloys [127]. Since the techniques employed in the papers $[126,127]$ differ in a number of details, more work will be necessary to clarify the origin of their different conclusions as well as to solve a number of open problems related to the effects of disorder in DMS [113].

\subsection{Two-dimensional ferromagnets}

Magnetism of epitaxial ultrathin transition-metal films on non-magnetic noble- or transitionmetal substrates has been studied intensively during the last two decades [128, 129]. Main differences with respect to bulk magnetism lie both in ground-state properties, where systematic DFT calculations predicted non-zero local moments also for other elements besides the five $3 d$ transition metals ( Cr, Mn, Fe, Co, Ni) [130, 131], and in finite-temperature behaviour, where the reduced dimensionality leads to a different universality class as compared to the bulk. In the limit of one-monolayer thickness of the film, one can realize a true two-dimensional magnet on a non-magnetic substrate. Equally interesting is the behaviour of magnetic properties with increasing film thickness, as can be documented by the intensively studied Fe films on an fcc $\mathrm{Cu}(001)$ substrate that display a variety of structures and magnetic configurations $[132,133]$. Their understanding in terms of ab initio techniques is a difficult task even concerning the ground-state properties [134].

The above formalism can easily be generalized to the two-dimensional case since the basic expression for the pair exchange interactions (11) is formulated in the real space. The magnetic properties resulting from a two-dimensional EHH can be obtained in a similar way like in the bulk case, see $(6,7,8,9)$, with the reciprocal-space vector $\mathbf{q}$ replaced by a two-dimensional vector $\mathbf{q}_{\|}$in the surface Brillouin zone (SBZ) and with the real-space sums restricted to lattice sites $\mathbf{R}, \mathbf{R}^{\prime}$ of the magnetic film. The site-offdiagonal blocks $g_{\mathbf{R}^{\prime}}^{\sigma}(z)$ of the Green function in (11) are determined using the surface Green function technique $[54,55]$, while the definition of $\Delta_{\mathbf{R}}(z)$ remains unchanged. The 
magnon energies are given by

$$
\begin{aligned}
E\left(\mathbf{q}_{\|}\right) & =\frac{4}{M}\left[J\left(\mathbf{0}_{\|}\right)-J\left(\mathbf{q}_{\|}\right)\right]+\Delta_{\text {an }}, \\
J\left(\mathbf{q}_{\|}\right) & =\sum_{\mathbf{R}} J_{\mathbf{0 R}} \exp \left(\mathbf{i} \mathbf{q}_{\|} \cdot \mathbf{R}\right),
\end{aligned}
$$

where $\Delta_{\text {an }}$ is a magnetic anisotropy energy which is a consequence of relativistic effects (spin-orbit interaction, magnetostatic dipole-dipole interaction). The Curie temperature in the MFA is given by (8) while the RPA leads to an expression

$$
\left(k_{\mathrm{B}} T_{\mathrm{C}}^{\mathrm{RPA}}\right)^{-1}=\frac{6}{M} \frac{1}{N_{\|}} \sum_{\mathbf{q}_{\|}} \frac{1}{E\left(\mathbf{q}_{\|}\right)},
$$

where $N_{\|}$is the number of $\mathbf{q}_{\|}$-vectors used in the SBZ-average.
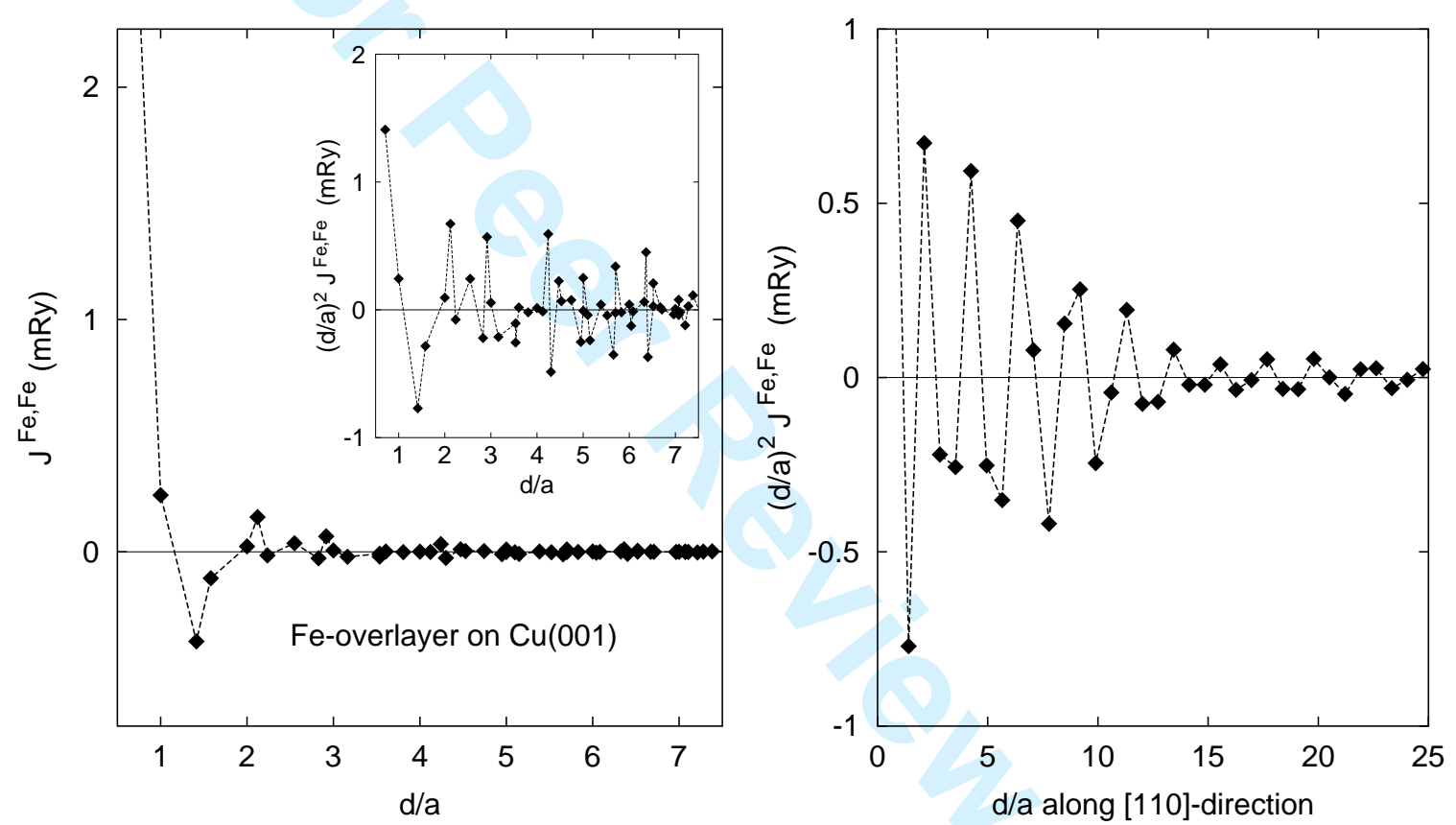

Figure 11: Exchange interactions $J_{\mathbf{R}^{\prime}}$ between Fe-moments in an Fe-overlayer on an fcc $\mathrm{Cu}(001)$ substrate as a function of the distance $\left|\mathbf{R}-\mathbf{R}^{\prime}\right|=d$ : interactions for all neighbours (left panel; the inset shows interactions multiplied by $d^{2}$ ) and interactions multiplied by $d^{2}$ for Fe atoms along the nearest-neighbour direction [110] (right panel).

The calculated pair exchange interactions $J_{\mathbf{R R}^{\prime}}$ in an Fe-monolayer on an fcc $\mathrm{Cu}(001)$ substrate are shown in figure 11 (left) as a function of the distance $d=\left|\mathbf{R}-\mathbf{R}^{\prime}\right|$. The first nearest-neighbour interaction dominates and the next-neighbour interactions exhibit an RKKY-like oscillatory behaviour with an envelope decaying proportionally to $d^{-2}$, in contrast to the bulk decay proportional to $d^{-3}$ (see inset and compare with figure 1). The complex character of the distance-dependence of $J_{\mathbf{R}^{\prime}}$ arises when interactions from all shells are displayed together similarly like in the bulk case. An expected oscillatory behaviour is resolved for pairs along a specific direction as illustrated in figure 11 (right). Note, however, that the present case is not strictly two-dimensional due to the indirect 
exchange interactions via the Cu-substrate; this fact is reflected by the RKKY-amplitude of $J_{\mathbf{R R}^{\prime}}$ decaying slightly faster than $d^{-2}$.

The indirect interaction between the magnetic atoms, which is mediated by the nonmagnetic atoms, has important consequences for magnetic properties of magnetic films placed on a non-magnetic substrate and covered by a non-magnetic cap-layer of a finite thickness. As reported in a recent experiment [135], the Curie temperature of fcc(001)-Fe ultrathin films on a $\mathrm{Cu}(001)$ substrate varies in a non-monotonous manner as a function of the $\mathrm{Cu}$ cap-layer thickness. Such a behaviour clearly cannot be explained within a localized picture of magnetism.

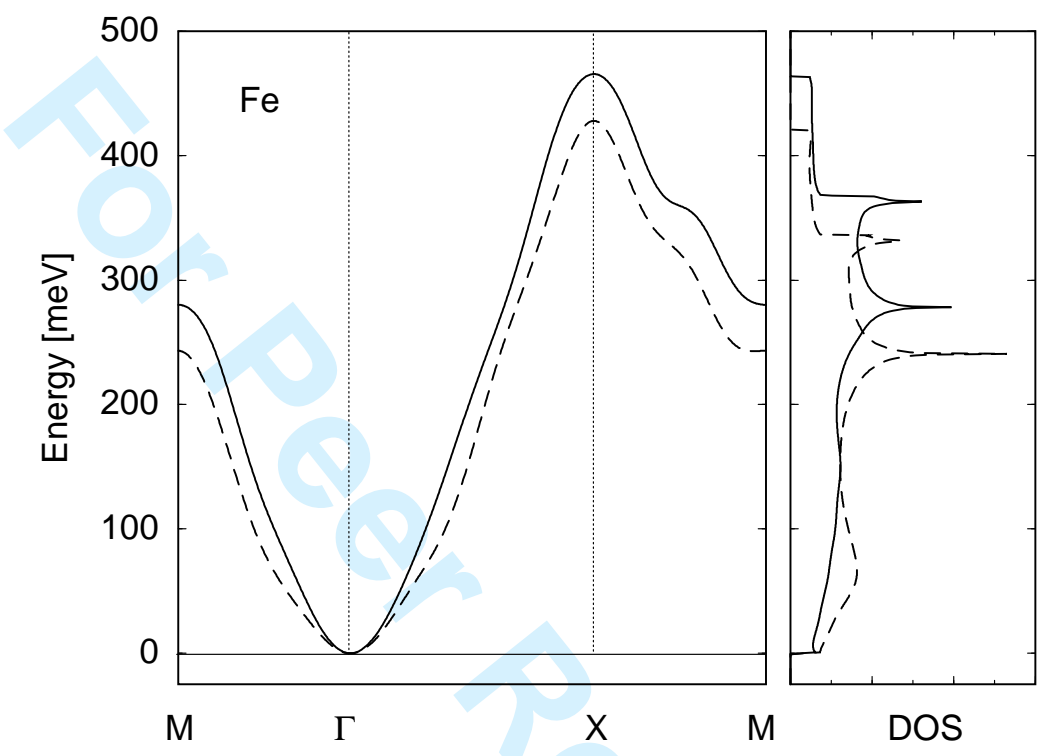

Figure 12: Magnon dispersion laws (left panel) and corresponding densities of states (right panel) for an Fe-layer embedded in fcc $\mathrm{Cu}$ (full lines) and an Fe-overlayer on fcc $\mathrm{Cu}(001)$ (dashed lines). We have set here $\Delta_{\text {an }}=0$ in (32).

Motivated by this finding we performed a systematic study of Fe- and Co-monolayers on an fcc $\mathrm{Cu}(001)$ substrate capped by another $\mathrm{Cu}$-layer of varying thickness $[14,16]$. Figure 12 presents the magnon spectra in two limiting cases, namely, for an uncovered Feoverlayer on $\mathrm{Cu}(001)$ and for an Fe-monolayer embedded in bulk $\mathrm{Cu}$, and figure 13 shows the full dependence of the magnetic moments and the first nearest-neighbour exchange interactions on the cap-layer thickness. The magnon spectra and the magnon densities of states exhibit all typical features of two-dimensional bands with the nearest-neighbour interactions which are here only slightly modified by non-vanishing interactions in next shells. The magnetic moments drop substantially on capping while their sensitivity to increasing cap-layer thickness is rather small. On the other hand, the behaviour of the nearest-neighbour exchange interaction is more complicated and it reflects interference effects in the $\mathrm{Cu}$-cap layer. The oscillations visible in right panel of figure 13 are due to quantum-well states in the $\mathrm{Cu}$-cap layer formed between the vacuum and the magnetic layer which, in turn, influence properties of the magnetic layer. Note that the values of the nearest-neighbour exchange interaction are significantly enhanced (roughly by a factor 2 or more) as compared to their bulk counterparts (cf. figure 1).

Calculations of the Curie temperatures of the two-dimensional ferromagnets represent 

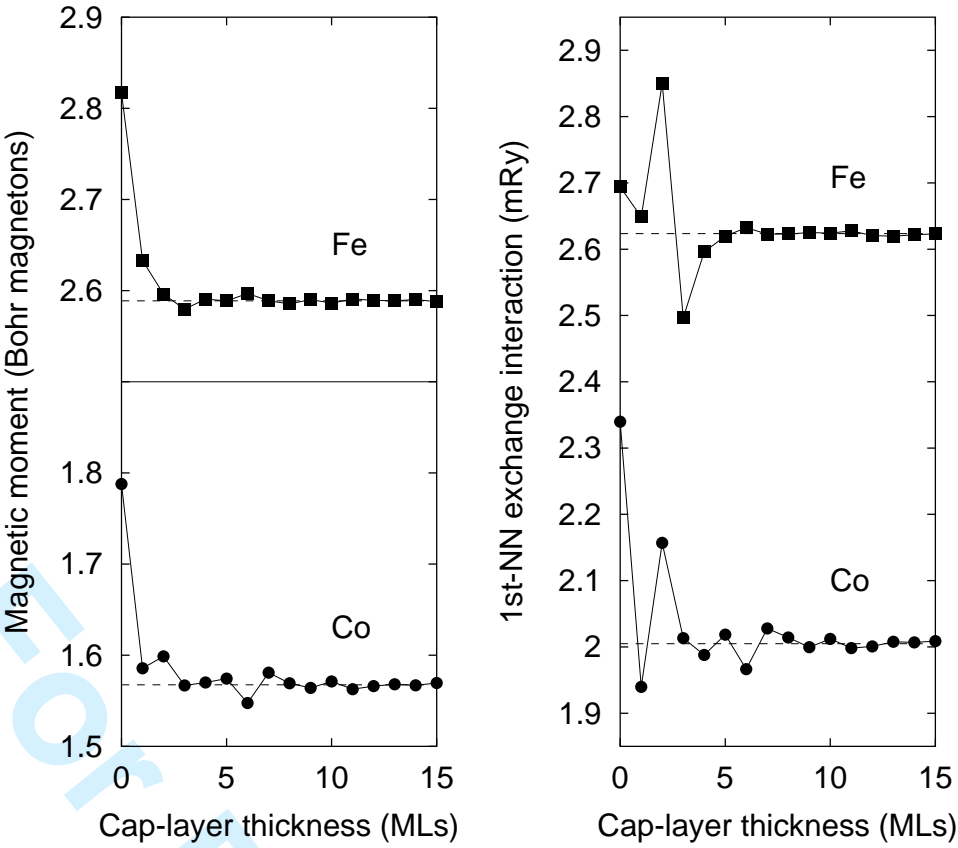

Figure 13: The local magnetic moments (left panel) and the first nearest-neighbour exchange interactions (right panel) in the Fe- and Co-monolayers on fcc $\mathrm{Cu}(001)$ as a function of the cap-layer thickness. The dashed lines represent the embedded layer limit (infinite cap thickness) while the limit of zero cap thickness corresponds to the uncovered overlayer.

a more difficult task than in the bulk case. The MFA Curie temperatures of the monolayers are typically of the same order of magnitude as the corresponding bulk temperatures [14] due to the fact that the reduced coordination is approximately compensated by the increase of the exchange interactions. This observation is in a strong disagreement with experimental data for ultrathin films which yield the Curie temperatures of the order $150-200 \mathrm{~K}$. This failure is due to the fact that the MFA violates the Mermin-Wagner theorem [136] due to the neglect of collective transverse fluctuations (spin waves) and it is thus inappropriate for two-dimensional systems.

Application of the RPA to the Curie temperature of a two-dimensional isotropic $\mathrm{EHH}$, $(32,33)$ with $\Delta_{\text {an }}=0$, yields a vanishing $T_{\mathrm{C}}^{\mathrm{RPA}}$ in agreement with the Mermin-Wagner theorem. Finite values of $T_{\mathrm{C}}^{\mathrm{RPA}}$ require non-zero values of the magnetic anisotropy energy $\Delta_{\text {an }}$ which is taken here as an adjustable parameter. This is not a serious problem as the RPA Curie temperature has only a weak logarithmic dependence upon $\Delta_{\text {an }}$ [137], and it is thus sufficient to know the order of magnitude of $\Delta_{\mathrm{an}}$. The latter is typically of the order of the dipolar energy $2 \pi\left(M \mu_{\mathrm{B}}\right)^{2} / V$, where $V$ is the atomic volume. In calculations we used $\Delta_{\text {an }}^{\mathrm{Co}}=0.052 \mathrm{mRy}$ and $\Delta_{\mathrm{an}}^{\mathrm{Fe}}=0.140 \mathrm{mRy}$.

The calculated RPA Curie temperatures are shown in figure 14 (left panel). They are strongly reduced as compared to the corresponding bulk values thereby improving on the MFA results. Nevertheless, they are still too large as compared to experiment. It is unclear whether this is due to some inaccuracy of the theory or to some imperfections of the samples used in experiments. On the other hand, such important experimental facts as the strong influence of the metallic coverage on the Curie temperature and the 


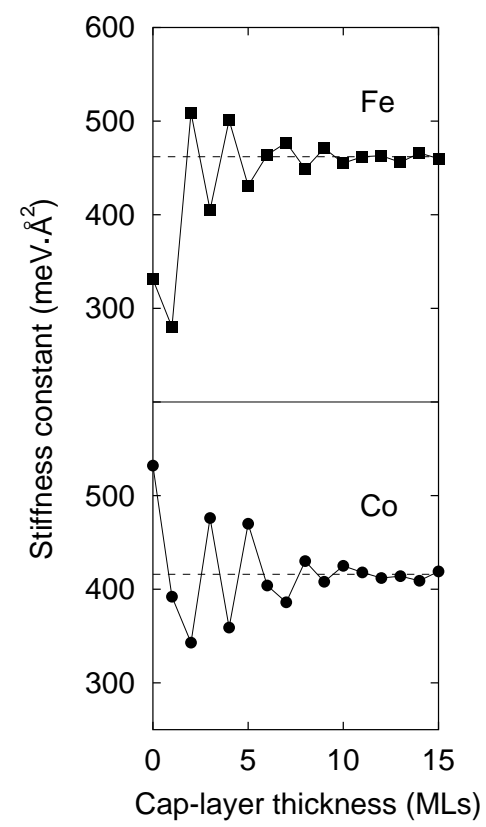

Figure 14: The Curie temperatures (left panel) and the spin-stiffness constants (right panel) of the Fe- and Co-monolayers on fcc $\mathrm{Cu}(001)$ as a function of the cap-layer thickness. The dashed lines represent the embedded layer limit (infinite cap thickness) while the limit of zero cap thickness corresponds to the uncovered overlayer case.

oscillations of the Curie temperature with the cap thickness [135] are well explained by the present theory. A more detailed analysis of the data reveals that the oscillations of the $T_{\mathrm{C}}^{\mathrm{RPA}}$ follow rather closely the behaviour of the spin-stiffness constants, see figure 14 (right panel). The similarity of both trends is due to the fact that the $T_{\mathrm{C}}^{\mathrm{RPA}}$ for a twodimensional system is determined predominantly by low-energy magnons.

It should be noted that an analogous oscillatory behaviour of the Curie temperature as a function of the non-magnetic spacer thickness has also been observed for fcc(001)$\mathrm{Co} / \mathrm{Cu} / \mathrm{Ni}$ trilayers [138]. The latter system has been investigated theoretically in terms of the on-site exchange parameters $J_{\mathbf{R}}^{0}[139]$.

\subsection{Surfaces of ferromagnets}

The surface of solids represents a playground for many novel, physically interesting phenomena not present in the bulk. At the same time, far from the surface the bulk behaviour is recovered. The knowlegde of exchange interactions at the system surface is the first necessary step towards a quantitative study of such properties like magnon spectra and critical temperatures. Corresponding experimental data for surfaces are very limited because reliable separation of the surface- and bulk-related features is a challenging problem. In such a situation the corresponding theoretical study is of a great importance, in particular in the framework of the parameter-free approach.

Reduced coordination at surfaces of transition-metal ferromagnets leads to an enhancement of zero-temperature surface magnetic moments over their bulk values [131]. For the FM hcp Gd, an enhancement of its Curie temperature at the (0001) surface over the bulk value was observed [140]. Theoretical explanation of the latter fact was provided 
by total-energy calculations using an LSDA+U approach [141]. An important role was ascribed to a small inward relaxation of the top surface layer. However, more recent works have thrown serious doubts on these conclusions, both on side of experiment [142] and theory [85].

We have recently performed calculations for low-index surfaces of bcc Fe, hcp Co, and hcp Gd [20] focused on layer-resolved local quantities like the magnetic moments and the on-site exchange parameters $J_{\mathbf{R}}^{0}(15)$. Note, however, that for inhomogeneous systems, such as surfaces, a direct relation between the Curie temperatures and the onsite exchange parameters $J_{\mathbf{R}}^{0}$ cannot be given. Hence, the latter quantities reflect merely the strength of the exchange interaction and its spatial variations in layered systems [139].
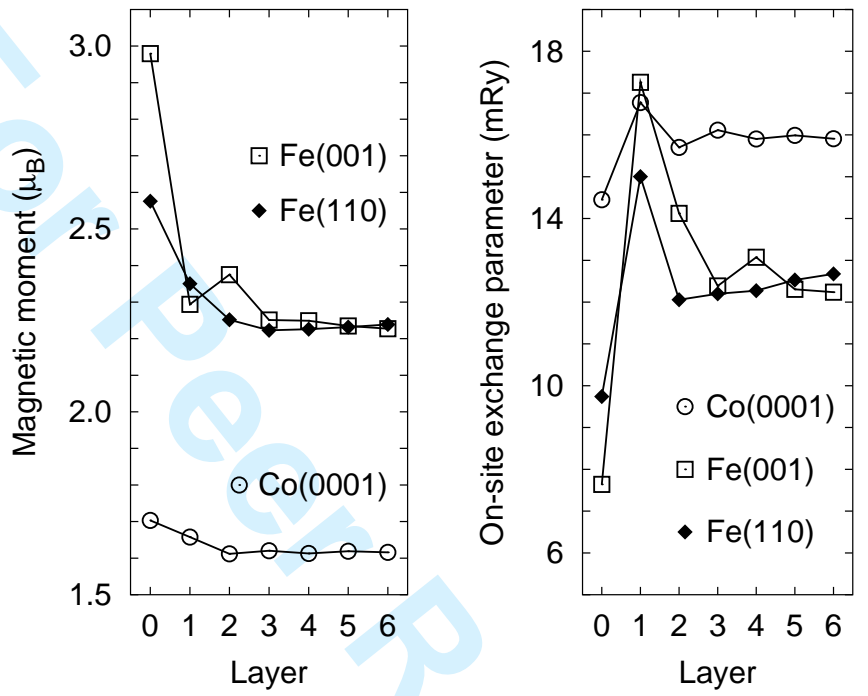

Figure 15: Layer-resolved magnetic moments (left panel) and on-site exchange parameters $J_{\mathbf{R}}^{0}$ (right panel) at surfaces of bcc Fe and hcp Co. The layer numbering starts from the top surface layer, denoted by 0 .

Figure 15 presents the results for Fe- and Co-surfaces. It is seen that the wellknown surface enhancement of the moments is accompanied by a more complicated layerdependence of the on-site exchange parameters exhibiting a minimum in the top surface layer and a maximum in the first subsurface layer. A qualitative explanation follows from $(11,15)$ which show that $J_{\mathbf{R}}^{0}$ reflects the exchange splitting on the $\mathbf{R}$-th site as well as the splittings and number of its neighbours. Hence, the reduction of $J_{\mathbf{R}}^{0}$ in the top surface layer is due to the reduced coordination, whereas the maximum in the first subsurface position is due to the full (bulk-like) coordination of these sites and the enhanced surface local moments, see figure 15. Note that the layer-dependence of the on-site exchange parameters and its explanation are analogous to the case of hyperfine magnetic fields at the nuclei of iron atoms $[54,143]$.

The $\operatorname{Gd}(0001)$ surface was treated in the 'open-core' approach mentioned in section 4.2; the results are presented in figure 16. Two models of the surface structure were used: with lattice sites occupying the ideal truncated bulk positions (unrelaxed structure) and with a $3 \%$ contraction of the interlayer separation between the two topmost atomic layers (inward relaxation). The magnitude of the contraction was set according to LEED measurements [144] and previous full-potential calculations [85]. The layer-resolved magnetic moments 


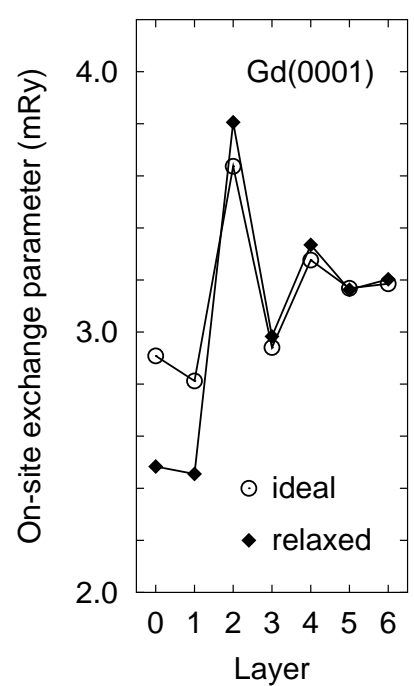

Figure 16: Layer-resolved magnetic moments (left panel) and on-site exchange parameters $J_{\mathbf{R}}^{0}$ (right panel) at the (0001) surface of hcp Gd as calculated with the lattice sites in the ideal truncated bulk positions and with the top surface layer relaxed towards the bulk. The layer numbering starts from the top surface layer, denoted by 0 .

exhibit a small surface enhancement followed by Friedel-like oscillations around the bulk value. These oscillations can be resolved also in the layer-dependences of the on-site exchange parameters $J_{\mathbf{R}}^{0}$ which, however, start with reduced values in the top surface layer due to the reduced coordination, as discussed for Fe- and Co-surfaces. The maximum of the on-site exchange parameters is found in the second subsurface layer, in contrast to the transition-metal surfaces, which can be explained by the reduced Gd-moments in the first subsurface layer. The surface relaxation does not modify investigated layer-dependences substantially: it leads to a small reduction of the local moments and the on-site exchange parameters in the first two top surface layers and a tiny enhancement in the second subsurface layer as compared to the ideal surface.

One can conclude that the surface enhancement of the local magnetic moments of the three FM metals is not accompanied by an analogous trend of on-site exchange parameters which might be an indication of a surface-induced enhancement of Curie temperatures. However, a calculation of the pair exchange interactions and an improved treatment of the EHH beyond the MFA remain important tasks for future.

\section{Discussion and outlook}

Exchange interactions and related quantities can be extracted from selfconsistent electronic structure calculations in a number of different ways; each of them has its own advantages and disadvantages. The simplest approach lies in obtaining the exchange interactions from total-energy differences calculated directly for various (usually collinear) spin configurations [85, 145, 146]. The usefulness of such schemes is, however, rather limited mainly due to a finite number of configurations used for the mapping. This fact together with the asymptotic behaviour of the exchange interactions $(12,13)$ make the simple mapping procedures suitable especially for non-metallic systems (half-metals, in- 
The central idea of mapping the infinitesimal changes of single-particle energies onto an effective classical Hamiltonian for localized spins has been used also for a quantitative description of effects beyond the bilinear isotropic exchange interaction in (2). Higher terms in expansion of the single-particle energies with respect to rotation angles give rise to biquadratic exchange interaction, important especially in metallic multilayers [48]. Inclusion of relativistic effects, leading to anisotropic exchange interaction and Dzialoshinskii-Moriya interaction, has been discussed for bulk systems [51, 148] and thin films [149]. In both cases, the gap in the spin-wave spectrum for zero wavevector can be calculated with accuracy better than used in section 4.5 for the quantity $\Delta_{\text {an }}$ in (32). The magnetic force theorem and expressions for effective interaction parameters have recently been formulated also for highly correlated systems treated beyond the LSDA [150].

It should be noted, however, that certain aspects of finite-temperature itinerant magnetism cannot be reproduced by effective Hamiltonians with one unit vector $\mathbf{e}_{\mathbf{R}}$ per lattice site only. An ab initio study based on a model Hamiltonian with bilinear, biquadratic and bicubic terms depending on the magnitude of local magnetic moments was formulated in reference [49]. The parametrization of the model was obtained from selfconsistent calculations for a number of spin-spiral states. The calculated Curie temperature of bcc Fe agrees well with experiment while the Curie temperatures of fcc Co and fcc Ni were underestimated by about $20-25 \%$.

Another ab initio approach based on effective Hamiltonians acting on more than one magnetic vector per atom was worked out in references $[69,84]$. It is based on a welldefined spatial separability of the $s p$ - and $d$-components of the spin density in late $3 d$ transition metals leading thus to two spin vectors per atom. Results of this approach for the magnon spectrum of fcc $\mathrm{Ni}$ seem to describe qualitatively well finer details in the experimental data around $100-150 \mathrm{meV}$ (sometimes denoted as an 'optical' magnon), which are manifestation of non-adiabatic effects [69]. In the case of heavy RE-metals ( $\mathrm{Gd}$ through Tm), three moment vectors per site were considered: the conduction electron $(s p d)$ spin moment vector and the $4 f$ electron spin and orbital moment vectors. The effective Hamiltonian in the latter case contains also intrasite spin-orbit coupling interaction and crystal-field terms. A first-principles approach to crystal-field parameters in RE-based systems can be found in references [82, 151].

As mentioned in section 2, the pair exchange interactions according to (5) do not contain contributions from constraining magnetic fields which appear as Lagrange multipliers in the constrained DFT. The accuracy of (5) has been recently addressed by several authors $[30,32,33]$. It has been shown that the pair interactions (5) obtained from the magnetic force theorem are appropriate for the dynamical response function (magnon spectrum) within the adiabatic time-dependent DFT, whereas a modification of the pair exchange interactions is desirable for the static response and related quantities [33]. In the latter case, the changes of the moment directions $\delta \mathbf{u}_{\mathbf{R}}$ and the constraining fields $\delta \mathbf{B}_{\mathbf{R}}^{\perp}$ satisfy linear relations [30]

$$
M_{\mathbf{R}} \delta \mathbf{u}_{\mathbf{R}}=\sum_{\mathbf{R}^{\prime}}\left(K_{\mathbf{R R}^{\prime}} \delta \mathbf{u}_{\mathbf{R}^{\prime}}+\chi_{\mathbf{R R}^{\prime}} \delta \mathbf{B}_{\mathbf{R}^{\prime}}^{\perp}\right)
$$

where $M_{\mathbf{R}}$ denotes the magnitude of $\mathbf{R}$-th local moment in units of $\mu_{\mathrm{B}}$ and where the exchange-correlation response function $K_{\mathbf{R R}^{\prime}}$ and the bare transverse susceptibility $\chi_{\mathbf{R R}^{\prime}}$ 


$$
\begin{aligned}
& K_{\mathbf{R R}^{\prime}}=\frac{2}{\pi} \operatorname{Im} \int_{-\infty}^{E_{\mathrm{F}}} d E \int_{\Omega_{\mathbf{R}}} d \mathbf{r} \int_{\Omega_{\mathbf{R}^{\prime}}} d \mathbf{r}^{\prime} G^{\uparrow}\left(\mathbf{r}, \mathbf{r}^{\prime} ; E^{+}\right) B_{\mathrm{xc}}\left(\mathbf{r}^{\prime}\right) \\
& \times G^{\downarrow}\left(\mathbf{r}^{\prime}, \mathbf{r} ; E^{+}\right), \\
& \chi_{\mathbf{R R}^{\prime}}=\frac{2}{\pi} \operatorname{Im} \int_{-\infty}^{E_{\mathrm{F}}} d E \int_{\Omega_{\mathbf{R}}} d \mathbf{r} \int_{\Omega_{\mathbf{R}^{\prime}}} d \mathbf{r}^{\prime} G^{\uparrow}\left(\mathbf{r}, \mathbf{r}^{\prime} ; E^{+}\right) \\
& \times G^{\downarrow}\left(\mathbf{r}^{\prime}, \mathbf{r} ; E^{+}\right) .
\end{aligned}
$$

As a consequence of non-zero constraining fields, the exchange parameters $J_{\mathbf{R R}^{\prime}}(5)$ get renormalized values given explicitly by [30]

$$
J_{\mathbf{R R}^{\prime}}^{\mathrm{ren}}=J_{\mathbf{R R}^{\prime}}-\frac{1}{2}\left\{\left(M-K^{T}\right) X^{-1}(M-K)\right\}_{\mathbf{R R}^{\prime}},
$$

where $M, K, X$ denote, respectively, matrices with elements $M_{\mathbf{R}} \delta_{\mathbf{R R}^{\prime}}, K_{\mathbf{R R}^{\prime}}, \chi_{\mathbf{R R}^{\prime}}$, and where $K^{T}$ denotes the transpose of $K$. The evaluation of $J_{\mathbf{R R}^{\prime}}^{\text {ren }}(36)$ for real systems remains yet to be performed.

Table 3: Curie temperature calculated within the RPA by using the bare $\left(T_{\mathrm{C}}^{\mathrm{RPA}}\right)$ and renormalized $\left(T_{\mathrm{C}}^{\mathrm{RPA}, \mathrm{ren}}\right)$ exchange interactions and their comparison with experimental values $\left(T_{\mathrm{C}, \exp }\right)$.

\begin{tabular}{lccr}
\hline Metal & $T_{\mathrm{C}}^{\mathrm{RPA}}[\mathrm{K}]$ & $T_{\mathrm{C}}^{\mathrm{RPA}, \mathrm{ren}}[\mathrm{K}]$ & \multicolumn{1}{c}{$T_{\mathrm{C}, \exp }[\mathrm{K}]$} \\
\hline Fe bcc & 950 & 1057 & $1044-1045$ \\
Co fcc & 1311 & 1771 & $1388-1398$ \\
Ni fcc & 350 & 634 & $624-631$ \\
\hline
\end{tabular}

Physical insight into the nature of this renormalization can be obtained for Bravais lattices assuming a sufficiently rigid magnetization within an atomic cell [30]. In such a case, the renormalization of the pair interactions (36) leads to a simple renormalization of the magnon energies $E(\mathbf{q})$ which is significant for high-energy magnons $E(\mathbf{q})>\Delta_{\text {ex }}$, where $\Delta_{\text {ex }}$ denotes an average exchange splitting. In particular, the spin-wave stiffness constant $D$ does not undergo any renormalization. The Curie temperature evaluated in the RPA (9) becomes renormalized according to a formula

$$
\left(k_{\mathrm{B}} T_{\mathrm{C}}^{\mathrm{RPA}, \mathrm{ren}}\right)^{-1}=\left(k_{\mathrm{B}} T_{\mathrm{C}}^{\mathrm{RPA}}\right)^{-1}-\frac{6}{M \Delta_{\mathrm{ex}}},
$$

which means that the renormalized Curie temperatures are enhanced as compared to the unrenormalized ones. The values for the bulk cubic $3 d$ ferromagnets are summarized in table 3. It can be seen that the renormalization of exchange parameters improves considerably the agreement between theory and experiment for Fe and $\mathrm{Ni}$ (see also [33]), in contrast to Co, where the unrenormalized Curie temperature is closer to experiment than the renormalized one. A quantitative analysis for bulk Gd shows that the effect of constraining fields is completely negligible [32]. 
As is well known, the exact magnon dispersion law is obtained from poles of the dynamical transverse susceptibility while the effective exchange interactions are closely related to the static transverse susceptibility [32,33]. Susceptibility calculations within the DFT are, however, quite involved even for systems with perfect three-dimensional translational symmetry $[152,52]$, which calls for additional approximations. The calculations can be simplified using again the adiabatic approximation and the ASA as done in reference [72]. The spin-wave spectrum of bcc Fe calculated from the susceptibility agrees very well with that from a frozen-magnon approach, whereas differences between the two approaches are found in results for fcc Co and fcc Ni [72]. However, for a correct reproduction of the 'optical' magnon branch of fcc Ni (including the lifetime effects), full susceptibility calculations [52] are inevitable.

Susceptibility calculations have been employed in another successful approach to finitetemperature magnetism, based on the DLM state $[26,47]$ the electronic structure of which is treated in the CPA. In contrast to the previous techniques starting from the magnetic ground state and its excitations, the DLM theory is focused on the paramagnetic state whereby no particular form of an effective spin Hamiltonian has to be assumed. The transition temperature is derived from divergence of the susceptibility of the DLM state which is related to a direct correlation function. An efficient evaluation of the latter quantity represents the most difficult part of computations. This mean-field technique has been applied to bulk bcc Fe and fcc Ni [80]; an especially good agreement between the calculated and experimental Curie temperatures has been achieved by including effects of correlations in terms of Onsager cavity fields. Applications to thin transition-metal films have been restricted to the mean-field level [153, 154]; the results for the Curie temperatures reproduce a number of experimentally observed trends with respect to the thickness of magnetic films and of non-magnetic capping layers. In the case of $\mathrm{Fe}$ and Co monolayers on a $\mathrm{Cu}(001)$ substrate, however, the theory predicts unrealistically high Curie temperatures, in full agreement with the MFA values from the EHH, see section 4.5 and reference [14].

The DLM state was also combined with the Heisenberg Hamiltonian $[155,156]$. The pair exchange interactions were derived within the CPA from band-energy changes between the parallel and antiparallel orientations of two particular magnetic moments assuming that the moments on other sites are randomly oriented. Application of this approach to bulk bcc Fe led to exchange interactions different from those presented in section 3.2: the first nearest-neighbour interaction from the DLM state is about three times bigger than the value shown in figure 1 . As a consequence, the Curie temperature, estimated within the spherical model (equivalent to the RPA), amounts to $T_{\mathrm{C}}=2700 \mathrm{~K}$ [155], substantially higher than the experimental and theoretical values in table 1. A very recent analysis [50] of this approach clarified the main reason for this overestimation: if the fully renormalized form of the exchange parameters $[155,156]$ is replaced by an unrenormalized one (corresponding to an infinitesimal rotation of magnetic moments described by the second-order term in an expansion of band-energy changes with respect to the CPAmedium), the resulting Curie temperatures come out in the range $T_{\mathrm{C}}=1090-1330 \mathrm{~K}$, i.e., only slightly higher than experiment. This comparison indicates that the two particular configurations of a pair of atomic moments (parallel and antiparallel) used for a quantitative analysis in Ref. [155] are too specific and should not be used for metallic systems. This conclusion is further corroborated by the original mean-field treatment of magnetic susceptibility of the DLM state 
The most important feature of the described ab initio approach to exchange interactions lies in its real-space formulation, which opens a way to study long-range interactions encountered in itinerant magnets including systems without three-dimensional translational invariance (random alloys, low-dimensional magnets). The inherent limitation to cases with large local magnetic moments makes the approach especially suitable for applications to systems as transition-metal surfaces and thin films, diluted magnetic semiconductors, rare-earth metals and compounds, etc.

\section{Acknowledgements}

We are grateful to L. Bergqvist, G. Bihlmayer, S. Blügel, G. Bouzerar, M. Diviš, P. Franek, F. Máca, O. Eriksson, M. Pajda, J. Rusz and P. Weinberger for collaboration at various stages of the project, and to P. Dederichs, B. Gyorffy, P. Levy, A. Liechtenstein, L. Sandratskii, A. Shick, L. Szunyogh and L. Udvardi for helpful discussions.

We acknowledge the financial support provided by the Academy of Sciences of the Czech Republic (A1010203, S2041105, AVOZ20410507, AVOZ10100520), the Ministry of Education of the Czech Republic (MSM0021620834), the Czech Science Foundation (202/04/0583, 202/05/2111), and the Center for Computational Materials Science in Vienna (GZ 45.547).

\section{References}

[1] P. Hohenberg and W. Kohn. Phys. Rev., 136:B864, 1964.

[2] W. Kohn and L. J. Sham. Phys. Rev., 140:A1133, 1965.

[3] U. von Barth and L. Hedin. J. Phys. C: Solid State Phys., 5:1629, 1972.

[4] J. B. Staunton. Rep. Progr. Phys., 57:1289, 1994.

[5] J. Kübler. Theory of Itinerant Electron Magnetism. Clarendon Press, Oxford, 2000.

[6] K. Baberschke, M. Donath, and W. Nolting, editors. Band-Ferromagnetism, volume 580 of Lecture Notes in Physics. Springer, Berlin, 2001.

[7] P. Mohn. Magnetism in the Solid State. Springer, Berlin, 2003.

[8] E. Runge and E. K. U. Gross. Phys. Rev. Lett., 52:997, 1984.

[9] N. D. Mermin. Phys. Rev., 137:A1441, 1965.

[10] A. I. Liechtenstein, M. I. Katsnelson, and V. A. Gubanov. J. Phys. F: Met. Phys., 14:L125, 1984.

[11] A. I. Liechtenstein, M. I. Katsnelson, V. P. Antropov, and V. A. Gubanov. J. Magn. Magn. Mater., 67:65, 1987.

[12] V. A. Gubanov, A. I. Liechtenstein, and A. V. Postnikov. Magnetism and the Electronic Structure of Crystals. Springer, Berlin, 1992. 
[13] V. P. Antropov, B. N. Harmon, and A. N. Smirnov. J. Magn. Magn. Mater., 200:148, 1999.

[14] M. Pajda, J. Kudrnovský, I. Turek, V. Drchal, and P. Bruno. Phys. Rev. Lett., 85:5424, 2000.

[15] M. Pajda, J. Kudrnovský, I. Turek, V. Drchal, and P. Bruno. Phys. Rev. B, 64:174402, 2001.

[16] P. Bruno, J. Kudrnovský, M. Pajda, V. Drchal, and I. Turek. J. Magn. Magn. Mater., 240:346, 2002.

[17] J. Kudrnovský, I. Turek, V. Drchal, F. Máca, J. Mašek, P. Weinberger, and P. Bruno. J. Superconductivity, 16:119, 2003.

[18] I. Turek, J. Kudrnovský, G. Bihlmayer, and S. Blügel. J. Phys.: Condens. Matter, 15:2771, 2003.

[19] I. Turek, J. Kudrnovský, M. Diviš, P. Franek, G. Bihlmayer, and S. Blügel. Phys. Rev. B, 68:224431, 2003.

[20] I. Turek, G. Bihlmayer, S. Blügel, and P. Weinberger. Czech. J. Phys., 53:81, 2003.

[21] G. Bouzerar, J. Kudrnovský, L. Bergqvist, and P. Bruno. Phys. Rev. B, 68:081203, 2003.

[22] J. Kudrnovský, I. Turek, V. Drchal, F. Máca, P. Weinberger, and P. Bruno. Phys. Rev. B, 69:115208, 2004.

[23] J. Kudrnovský, I. Turek, V. Drchal, and P. Bruno. J. Magn. Magn. Mater., 272276:1995, 2004.

[24] L. Bergqvist, O. Eriksson, J. Kudrnovský, V. Drchal, P. Korzhavyi, and I. Turek. Phys. Rev. Lett., 93:137202, 2004.

[25] S. Khmelevskyi, J. Kudrnovský, B. L. Gyorffy, P. Mohn, V. Drchal, and P. Weinberger. Phys. Rev. B, 70:224432, 2004.

[26] B. L. Gyorffy, A. J. Pindor, J. Staunton, G. M. Stocks, and H. Winter. J. Phys. F: Met. Phys., 15:1337, 1985.

[27] P. H. Dederichs, S. Blügel, R. Zeller, and H. Akai. Phys. Rev. Lett., 53:2512, 1984.

[28] L. M. Sandratskii. Adv. Phys., 47:91, 1998.

[29] L. M. Sandratskii. J. Phys.: Condens. Matter, 3:8565, 1991.

[30] P. Bruno. Phys. Rev. Lett., 90:087205, 2003.

[31] A. Oswald, R. Zeller, P. J. Braspenning, and P. H. Dederichs. J. Phys. F: Met. Phys., 15:193, 1985.

[32] V. P. Antropov. J. Magn. Magn. Mater., 262:L192, 2003. 
[33] M. I. Katsnelson and A. I. Lichtenstein. J. Phys.: Condens. Matter, 16:7439, 2004.

[34] S. V. Halilov, H. Eschrig, A. Y. Perlov, and P. M. Oppeneer. Phys. Rev. B, 58:293, 1998.

[35] V. P. Antropov, M. I. Katsnelson, M. van Schilfgaarde, and B. N. Harmon. Phys. Rev. Lett., 75:729, 1995.

[36] V. P. Antropov, M. I. Katsnelson, B. N. Harmon, M. van Schilfgaarde, and D. Kusnezov. Phys. Rev. B, 54:1019, 1996.

[37] Q. Niu and L. Kleinman. Phys. Rev. Lett., 80:2205, 1998.

[38] Q. Niu, X. Wang, L. Kleinman, W.-M. Liu, D. M. C. Nicholson, and G. M. Stocks. Phys. Rev. Lett., 83:207, 1999.

[39] S. V. Tyablikov. Methods of Quantum Theory of Magnetism. Plenum Press, New York, 1967.

[40] C. S. Wang, R. E. Prange, and V. Korenman. Phys. Rev. B, 25:5766, 1982.

[41] E. Şaşığlu, L. M. Sandratskii, and P. Bruno. Phys. Rev. B, 70:024427, 2004.

[42] J. Rusz, I. Turek, and M. Diviš. Phys. Rev. B, 71:174408, 2005.

[43] J. Fröhlich, B. Simon, and T. Spencer. Commun. Math. Phys., 50:79, 1976.

[44] Y. G. Sinai. Theory of Phase Transitions: Rigorous Results. Pergamon Press, Oxford, 1982.

[45] T. Moryia, editor. Electron Correlation and Magnetism in Narrow-Band Systems. Springer, Berlin, 1981.

[46] T. Moryia. Spin Fluctuations in Itinerant Electron Magnetism. Springer, Berlin, 1985.

[47] J. B. Staunton, S. S. A. Razee, L. Szunyogh, and B. L. Gyorffy. Physica B, 318:316, 2002.

[48] O. N. Mryasov, A. J. Freeman, and A. I. Liechtenstein. J. Appl. Phys., 79:4805, 1996.

[49] N. M. Rosengaard and B. Johansson. Phys. Rev. B, 55:14975, 1997.

[50] A. V. Ruban, S. Shallcross, S. I. Simak, and H. L. Skriver. Phys. Rev. B, 70:125115, 2004.

[51] V. P. Antropov, M. I. Katsnelson, and A. I. Liechtenstein. Physica B, 237-238:336, 1997.

[52] S. Y. Savrasov. Phys. Rev. Lett., 81:2570, 1998.

[53] O. K. Andersen and O. Jepsen. Phys. Rev. Lett., 53:2571, 1984. 
Philosophical Magazine \& Philosophical Magazine Letters

[54] I. Turek, V. Drchal, J. Kudrnovský, M. Sob, and P. Weinberger. Electronic Structure of Disordered Alloys, Surfaces and Interfaces. Kluwer, Boston, 1997.

[55] I. Turek, J. Kudrnovský, and V. Drchal. In H. Dreyssé, editor, Electronic Structure and Physical Properties of Solids, volume 535 of Lecture Notes in Physics, page 349. Springer, Berlin, 2000.

[56] S. H. Vosko, L. Wilk, and M. Nusair. Can. J. Phys., 58:1200, 1980.

[57] P. Weinberger. Electron Scattering Theory for Ordered and Disordered Matter. Clarendon Press, Oxford, 1990.

[58] A. Gonis. Green Functions for Ordered and Disordered Systems. North-Holland, Amsterdam, 1992.

[59] O. Gunnarsson, O. Jepsen, and O. K. Andersen. Phys. Rev. B, 27:7144, 1983.

[60] I. Turek, J. Kudrnovský, V. Drchal, P. Bruno, and S. Blügel. Physica Status Solidi $B, 236: 318,2003$.

[61] E. Sjöstedt and L. Nordström. Phys. Rev. B, 66:014447, 2002.

[62] P. Bruno. Phys. Rev. B, 52:411, 1995.

[63] J. Mathon. Phys. Rev. B, 27:1916, 1983.

[64] K. C. Hass, B. Velický, and H. Ehrenreich. Phys. Rev. B, 29:3697, 1984.

[65] D. Spišák and J. Hafner. J. Magn. Magn. Mater., 168:257, 1997.

[66] R. F. Sabiryanov, S. K. Bose, and O. N. Mryasov. Phys. Rev. B, 51:8958, 1995.

[67] S. Frota-Pessoa, R. B. Muniz, and J. Kudrnovský. Phys. Rev. B, 62:5293, 2000.

[68] L. Szunyogh and L. Udvardi. J. Magn. Magn. Mater., 198-199:537, 1999.

[69] S. Halilov. In D. J. Singh and D. A. Papaconstantopoulos, editors, Electronic Structure and Magnetism of Complex Materials, volume 54 of Springer Series in Materials Science, chapter 1, page 1. Springer, Berlin, 2003.

[70] M. van Schilfgaarde and V. P. Antropov. J. Appl. Phys., 85:4827, 1999.

[71] L. M. Sandratskii and P. Bruno. Phys. Rev. B, 66:134435, 2002.

[72] O. Grotheer, C. Ederer, and M. Fähnle. Phys. Rev. B, 63:100401(R), 2001.

[73] C. K. Loong, J. M. Carpenter, J. W. Lynn, R. A. Robinson, and H. A. Mook. J. Appl. Phys., 55:1895, 1984.

[74] J. W. Lynn. Phys. Rev. B, 11:2624, 1975.

[75] H. A. Mook and D. McK. Paul. Phys. Rev. Lett., 54:227, 1985.

[76] G. Shirane, V. J. Minkiewicz, and R. Nathans. J. Appl. Phys., 39:383, 1968. 
[77] H. A. Mook, J. W. Lynn, and M. R. Nicklow. Phys. Rev. Lett., 30:556, 1973.

[78] R. Pauthenet. J. Appl. Phys., 53:2029 and 8187, 1982.

[79] R. H. Brown, D. M. C. Nicholson, X. Wang, and T. C. Schulthess. J. Appl. Phys., 85:4830, 1999.

[80] J. B. Staunton and B. L. Gyorffy. Phys. Rev. Lett., 69:371, 1992.

[81] M. Heinemann and W. M. Temmerman. Phys. Rev. B, 49:4348, 1994.

[82] M. Richter. J. Phys. D: Appl. Phys., 31:1017, 1998.

[83] M. S. S. Brooks, L. Nordström, and B. Johansson. J. Phys.: Condens. Matter, 3:2357, 1991.

[84] A. Y. Perlov, S. V. Halilov, and H. Eschrig. Phys. Rev. B, 61:4070, 2000.

[85] P. Kurz, G. Bihlmayer, and S. Blügel. J. Phys.: Condens. Matter, 14:6353, 2002.

[86] P. Strange, A. Svane, W. M. Temmerman, Z. Szotek, and H. Winter. Nature, 399:756, 1999.

[87] P. A. Lindgard. Phys. Rev. B, 17:2348, 1978.

[88] N. G. Nereson, C. E. Olsen, and G. P. Arnold. Phys. Rev., 135:A176, 1964.

[89] A. H. Millhouse and K. A. McEwen. Solid State Commun., 13:339, 1973.

[90] P. Franek. Substitutional disorder in systems with correlated f-electrons. Master's thesis, Charles University, Prague, 2003.

[91] J. Jensen and A. R. Mackintosh. Rare Earth Magnetism. Clarendon, Oxford, 1991.

[92] M. S. S. Brooks and B. Johansson. In K. H. J. Buschow, editor, Handbook of Magnetic Materials, volume 7, chapter 3, page 139. North-Holland, Amsterdam, 1993.

[93] J. Kuneš and R. Laskowski. Phys. Rev. B, 70:174415, 2004.

[94] J. Kudrnovský and V. Drchal. Phys. Rev. B, 41:7515, 1990.

[95] P. Bruno, J. Kudrnovský, V. Drchal, and I. Turek. Phys. Rev. Lett., 76:4254, 1996.

[96] J. Kudrnovský, V. Drchal, I. Turek, P. Bruno, P. Dederichs, and P. Weinberger. In H. Dreyssé, editor, Electronic Structure and Physical Properties of Solids, volume 535 of Lecture Notes in Physics, page 313. Springer, Berlin, 2000.

[97] A. I. Liechtenstein, V. P. Antropov, and V. A. Gubanov. Physics of Metals and Metallography, 64:(4) 35, 1987.

[98] P. M. Levy, S. Maekawa, and P. Bruno. Phys. Rev. B, 58:5588, 1998. 
[99] A. V. Ruban, M. I. Katsnelson, W. Olovsson, S. I. Simak, and I. A. Abrikosov. Phys. Rev. B, 71:054402, 2005.

[100] J. A. Blackman and R. J. Elliott. J. Phys. C: Solid State Phys., 2:1670, 1969.

[101] V. I. Anisimov, V. P. Antropov, A. I. Liechtenstein, V. A. Gubanov, and A. V. Postnikov. Phys. Rev. B, 37:5598, 1988.

[102] S. D. Drell and L. Verlet. Phys. Rev., 99:849, 1955.

[103] K. Binder and A. P. Young. Rev. Mod. Phys., 58:801, 1986.

[104] M. F. Ling, J. B. Staunton, and D. D. Johnson. J. Phys.: Condens. Matter, 6:5981, 1994.

[105] M. F. Ling, J. B. Staunton, and D. D. Johnson. Europhys. Lett., 25:631, 1994.

[106] M. F. Ling, J. B. Staunton, and D. D. Johnson. J. Phys.: Condens. Matter, 6:6001, 1994.

[107] M. F. Ling, J. B. Staunton, D. D. Johnson, and F. J. Pinski. Phys. Rev. B, 52:R3816, 1995.

[108] G. Bouzerar and P. Bruno. Phys. Rev. B, 66:014410, 2002.

[109] S. Ghosh, P. L. Leath, and M. H. Cohen. Phys. Rev. B, 66:214206, 2002.

[110] G. Bouzerar, T. Ziman, and J. Kudrnovský. Europhys. Lett., 69:812, 2005.

[111] H. Akai. Phys. Rev. Lett., 81:3002, 1998.

[112] H. Ohno. J. Magn. Magn. Mater., 200:110, 1999.

[113] C. Timm. J. Phys.: Condens. Matter, 15:R1865, 2003.

[114] K. W. Edmonds et al. Phys. Rev. Lett., 92:037201, 2004.

[115] K. W. Edmonds et al. Appl. Phys. Lett., 81:4991, 2002.

[116] F. Máca and J. Mašek. Phys. Rev. B, 65:235209, 2002.

[117] P. A. Korzhavyi et al. Phys. Rev. Lett., 88:187202, 2002.

[118] O. Eriksson, L. Bergqvist, B. Sanyal, J. Kudrnovský, V. Drchal, P. Korzhavyi, and I. Turek. J. Phys.: Condens. Matter, 16:S5481, 2004.

[119] K. Sato, P. H. Dederichs, and H. Katayama-Yoshida. Europhys. Lett., 61:403, 2003.

[120] K. Binder and D. W. Heermann. Monte Carlo Simulation in Statistical Physics. Springer, Berlin, 2002.

[121] K. Sato, W. Schweika, P. H. Dederichs, and H. Katayama-Yoshida. Phys. Rev. B, 70:201202(R), 2004. 
[122] K. Sato, P. H. Dederichs, H. Katayama-Yoshida, and J. Kudrnovský. Physica B, 340-342:863, 2003.

[123] L. M. Sandratskii, P. Bruno, and J. Kudrnovský. Phys. Rev. B, 69:195203, 2004.

[124] A. B. Shick, J. Kudrnovský, and V. Drchal. Phys. Rev. B, 69:125207, 2004.

[125] L. Bergqvist, O. Eriksson, J. Kudrnovský, V. Drchal, A. Bergman, L. Nordström, and I. Turek. Phys. Rev. B, 72:(in print-BC10114), 2005.

[126] J. L. Xu, M. van Schilfgaarde, and G. D. Samolyuk. Phys. Rev. Lett., 94:097201, 2005.

[127] G. Bouzerar, T. Ziman, and J. Kudrnovský. Appl. Phys. Lett., 85:4941, 2004.

[128] H. C. Siegmann. J. Phys.: Condens. Matter, 4:8395, 1992.

[129] P. Poulopoulos and K. Baberschke. J. Phys.: Condens. Matter, 11:9495, 1999.

[130] A. J. Freeman and R. Wu. J. Magn. Magn. Mater., 100:497, 1991.

[131] M. Weinert and S. Blügel. In L. H. Bennett and R. E. Watson, editors, Magnetic Multilayers, page 51. World Scientific, Singapore, 1994.

[132] M. Straub, R. Vollmer, and J. Kirschner. Phys. Rev. Lett., 77:743, 1996.

[133] D. Qian, X. F. Jin, J. Barthel, M. Klaua, and J. Kirschner. Phys. Rev. Lett., $87: 227204,2001$.

[134] T. Asada and S. Blügel. Phys. Rev. Lett., 79:507, 1997.

[135] R. Vollmer, S. van Dijken, M. Schleberger, and J. Kirschner. Phys. Rev. B, 61:1303, 2000 .

[136] N. D. Mermin and H. Wagner. Phys. Rev. Lett., 17:1133, 1966.

[137] P. Bruno. Mater. Res. Soc. Symp. Proc., 231:299, 1992.

[138] A. Ney, F. Wilhelm, M. Farle, P. Poulopoulos, P. Srivastava, and K. Baberschke. Phys. Rev. B, 59:R3938, 1999.

[139] E. I. Isaev, L. V. Pourovskii, A. M. N. Niklasson, Yu. Kh. Vekilov, B. Johansson, and I. A. Abrikosov. Phys. Rev. B, 65:024435, 2001.

[140] E. D. Tober et al. Phys. Rev. Lett., 81:2360, 1998.

[141] A. B. Shick, W. E. Pickett, and C. S. Fadley. Phys. Rev. B, 61:R9213, 2000.

[142] C. S. Arnold and D. P. Pappas. Phys. Rev. Lett., 85:5202, 2000.

[143] S. Blügel. In Magnetismus von Festkörpern und Grenzflächen. Forschungszentrum Jülich, 1993. 
[145] D. Spišák and J. Hafner. Phys. Rev. B, 65:235405, 2002.

[146] D. Ködderitzsch, W. Hergert, W. M. Temmerman, Z. Szotek, A. Ernst, and H. Winter. Phys. Rev. B, 66:064434, 2002.

[147] P. Novák and J. Rusz. Phys. Rev. B, 71:184433, 2005.

[148] V. P. Antropov and A. I. Liechtenstein. Mater. Res. Soc. Symp. Proc., 253:325, 1992.

[149] L. Udvardi, L. Szunyogh, K. Palotás, and P. Weinberger. Phys. Rev. B, 68:104436, 2003.

[150] M. I. Katsnelson and A. I. Lichtenstein. Phys. Rev. B, 61:8906, 2000.

[151] P. Novák and J. Kuriplach. Phys. Rev. B, 50:2085, 1994.

[152] J. Callaway, A. K. Chatterjee, S. P. Singhal, and A. Ziegler. Phys. Rev. B, 28:3818, 1983.

[153] S. S. A. Razee, J. B. Staunton, L. Szunyogh, and B. L. Gyorffy. Phys. Rev. B, 66:094415, 2002.

[154] S. S. A. Razee, J. B. Staunton, L. Szunyogh, and B. L. Gyorffy. Phys. Rev. Lett., 88:147201, 2002.

[155] T. Oguchi, K. Terakura, and N. Hamada. J. Phys. F: Met. Phys., 13:145, 1983.

[156] T. Oguchi, K. Terakura, and A. R. Williams. Phys. Rev. B, 28:6443, 1983. 